\title{
Rethinking Individualism and Collectivism: Evaluation of Theoretical Assumptions and Meta-Analyses
}

\author{
Daphna Oyserman, Heather M. Coon, and Markus Kemmelmeier \\ University of Michigan
}

\begin{abstract}
Are Americans more individualistic and less collectivistic than members of other groups? The authors summarize plausible psychological implications of individualism-collectivism (IND-COL), metaanalyze cross-national and within-United States IND-COL differences, and review evidence for effects of IND-COL on self-concept, well-being, cognition, and relationality. European Americans were found to be both more individualistic - valuing personal independence more - and less collectivistic - feeling duty to in-groups less - than others. However, European Americans were not more individualistic than African Americans, or Latinos, and not less collectivistic than Japanese or Koreans. Among Asians, only Chinese showed large effects, being both less individualistic and more collectivistic. Moderate IND-COL effects were found on self-concept and relationality, and large effects were found on attribution and cognitive style.
\end{abstract}

To contemporary Americans, being an individualist is not only a good thing; it is a quintessentially American thing. However, the term individualism itself appears to have its roots outside of the North American continent, namely in the French Revolution. It appears that individualism was first used to describe the negative influence of individual rights on the well-being of the commonwealth. The rising tide of the individual rights movement was feared; it was thought that individualism would soon make community "crumble away, be disconnected into the dust and powder of individuality" (Burke, 1790/1973, p. 109). In this usage, individualism describes a worldview antagonistic to community and collective social structure.

Indeed, there is a long Western tradition of contrasting individual and collective focus. For example, Emile Durkheim (1887/ 1933) used the terms organic and mechanical solidarity to contrast the temporary relations formed in complex societies among dissimilar others (organic solidarity) - an individual focus - and the

Daphna Oyserman, Department of Psychology, School of Social Work, and Institute for Social Research, University of Michigan; Heather M. Coon and Markus Kemmelmeier, Department of Psychology, University of Michigan.

Heather M. Coon is now at the Department of Psychology, North Central College. Markus Kemmelmeier is now at the Department of Sociology, University of Nevada, Reno.

Daphna Oyserman was supported by a Research Scholar's Award from the W. T. Grant Foundation while writing this article; this support and the support of the Center for Advanced Studies in the Behavioral Sciences are gratefully acknowledged. A Founders' Dissertation Fellowship from the Institute of Social Research and the Department of Psychology, University of Michigan, supported Markus Kemmelmeier. We thank the participants of the 1998 Stanford Mini-Conference, the Stanford culture lab, Jennifer Boyd Ritsher, Hazel Markus, Tony Manstead, Agneta Fischer, and Norbert Schwarz for their comments on earlier versions of this article.

Correspondence concerning this article should be addressed to Daphna Oyserman, Institute for Social Research, Room 5240, University of Michigan, 426 Thompson Avenue, Ann Arbor, Michigan 48106-1248. E-mail: daphna.oyserman@umich.edu permanent bonds formed among similar others in traditional societies (mechanical solidarity) - a collective focus. Max Weber (1930) contrasted individual-focused Western European Protestantism with collective-focused Catholicism. He saw the former as promoting self-reliance and pursuit of personal interests and the latter as promoting permanent and hierarchical relationships. Similarly, Tönnies (1887/1957) contrasted the community-focused (Gemeinschaft) relationships of small villages with the association-based (Gesellschaft) relationships of urban societies.

In the past 20 years, the idea of contrasting societies on the basis of differences in individualism has increased in popularity, in large part because of the highly influential work of Geert Hofstede. In his widely cited book Culture's Consequences, Hofstede (1980) differentiated country-level individualism from "power distance," "masculinity," and "uncertainty avoidance." Within his conceptualization, the specific questions used to assess individualism focused on the workplace, contrasting the extent that workers valued personal time and choice with the extent they valued job security and on-the-job training. Hofstede (1980) reviewed possible antecedents and implications of these job-relevant values for societies. Although certainly not the first social scientist to focus explicitly on culture, Hofstede's model was important because it organized cultural differences into overarching patterns, which facilitated comparative research and launched a rapidly expanding body of cultural and cross-cultural research in the ensuing 20 years. Because of Hofstede's influence in organizing culture research around the concept of individualism, the present review focuses on research published since 1980 .

Usually, researchers conceptualize individualism as the opposite of collectivism (e.g., Hui, 1988), especially when contrasting European American and East Asian cultural frames (e.g., Chan, 1994; Kitayama, Markus, Matsumoto, \& Norasakkunkit, 1997; Yamaguchi, 1994). Social scientists assume that individualism is more prevalent in industrialized Western societies than in other societies, especially more traditional societies in developing countries. Protestantism and the process of civic emancipation in Western societies resulted in social and civic structures that championed the 
role of individual choice, personal freedom, and self-actualization (e.g., Inglehart, 1997; Sampson, 2001). Researchers assume that these processes led to a Western cultural focus on individualism that is more salient in countries and ethnic groups with a Protestant heritage, applying the idea of Western individualism to both crossregional and within-country comparisons of ethnic groups with different cultural heritages. Thus, within the United States it is commonly assumed that European Americans are higher in individualism and lower in collectivism than are members of ethnic minority groups (e.g., Freeberg \& Stein, 1996; Gaines et al., 1997; Rhee, Uleman, \& Lee, 1996). Taken together, current theorizing in cultural psychology portrays European Americans as the most individualistic group.

Considering European Americans the gold standard of individualism seems at first glance unremarkable. Certainly, "rugged individualism" has been an American hallmark at least since de Tocqueville's (1835/1969) classic analysis of America that linked individualism with individual rights and freedom, equal opportunity, and limited government (Lukes, 1973). Others have also linked American individualism to the Puritans, the founding fathers, the birth of a market economy, and the vast American frontier (Curry \& Valois, 1991). From the beginning, Americans have been enjoined to value "life, liberty, and the pursuit of happiness" and to think of themselves as separate and independent individuals, isolated from others. In de Tocqueville's words, "Such folk owe no man anything and hardly expect anything from anybody. They form the habit of thinking of themselves in isolation and imagine that their whole destiny is in their own hands" (1835/1969, p. 508).

Indeed, modern American cultural icons continue to articulate this belief in individualism. Personal privacy, individual rights, and personal freedoms are extolled; personal pleasure and autonomy are valued; and every American is exhorted to create a personal, private, and unique self (e.g., Sampson, 1977, 1988). Americans celebrate individualism as a uniquely American characteristic, an integral part of their culture. Yet, in spite of the seeming consensus that European Americans are the prototype defining individualism, we are aware of no systematic test of the underlying assumption that European Americans value or behave more individualistically than others.

Furthermore, there is a clear tension between the assumption that European Americans are uniquely high in individualism and low in collectivism and the assumption that the psychological models developed within this cultural frame - of self-concept content and functioning, well-being, attribution style, and relationality-are universal models, not simply models derived from and applicable to an individualistic worldview. Therefore, the basic aim of the present review is to address two questions: (a) Are European Americans higher in individualism and lower in collectivism than people from other societies? (b) Are theoretically derived implications of individualism and collectivism for psychological functioning borne out in the empirical literature? To answer these questions, first we present an overview of the theoretical implications of individualism and collectivism for basic psychological domains. Second, we provide a guide to how psychologists have studied individualism and collectivism. Third, we metaanalyze empirical literature assessing individualism and collectivism. Fourth, we review the empirical evidence of an influence of individualism and collectivism on basic psychological domains (self-concept, well-being, attribution style, and relationality). Last, we draw conclusions and articulate emerging questions about implications of a culture frame for psychology generally.

Methodologically, we collected all English-language literature published since 1980 on individualism and collectivism that either assessed these constructs directly or related them to the basic psychological domains of self-concept, well-being, attribution style, or relationality. We meta-analyzed individualism and collectivism studies contrasting European Americans with other groups and reviewed the empirical literature linking individualism and collectivism with basic psychological processes. Together the meta-analyses and literature reviews clarify the extent to which European Americans are in fact uniquely high in individualism and make sense of themselves, their lives, and their relationships with others in terms of the values of individualism.

As we demonstrate in subsequent sections, contemporary American psychological research is particularly suited to an individualistic worldview and may not necessarily fit as a universal model of human behavior to the extent that other peoples or regions of the world are sharply different from Americans in individualism and collectivism. For example, self-concept research is dominated both by a focus on self-esteem and by the belief that attainment of personal happiness is a basic motivational drive (e.g., Baumeister, 1998). Likewise, person perception and cognitive processes are understood in terms of stable traits, and equity is viewed as the basis for successful relationships (e.g., Triandis, 1995). These research frames fit individualistic, not collectivistic, conceptions of human nature. To preview our findings, our analysis of the literature suggests first that differences in individualism do exist and second that the influence of cultural frame is better documented for the domains of relationality and attribution than for other domains.

\section{CULTURAL ORIENTATIONS: DEFINITIONS AND PSYCHOLOGICAL CONSEQUENCES}

In this section, we provide an overview of individualism and collectivism as cultural orientations to provide a framework for reading the article as a whole.

\section{Individualism}

The core element of individualism is the assumption that individuals are independent of one another. From this core, a number of plausible consequences or implications of individualism can be discerned. One question we explore further is whether research has empirically validated these plausible consequences or implications and whether these plausible consequences are, in fact, universally part of individualism.

Hofstede (1980) defined individualism as a focus on rights above duties, a concern for oneself and immediate family, an emphasis on personal autonomy and self-fulfillment, and the basing of one's identity on one's personal accomplishments. Waterman (1984) defined normative individualism as a focus on personal responsibility and freedom of choice, living up to one's potential, and respecting the integrity of others. Schwartz (1990) defined individualistic societies as fundamentally contractual, consisting of narrow primary groups and negotiated social relations, with specific obligations and expectations focusing on achieving 
status. These definitions all conceptualize individualism as a worldview that centralizes the personal-personal goals, personal uniqueness, and personal control-and peripheralizes the social (Bellah, Madsen, Sullivan, Swidler, \& Tipton, 1985; Hsu, 1983; Kagitcibasi, 1994; U. Kim, 1994; Markus \& Kitayama, 1991; Sampson, 1977; Triandis, 1995).

Given these definitions, plausible consequences of individualism for psychology-self-concept, well-being, attribution style, and relationality - are easily discerned. First, with regard to selfconcept, individualism implies that (a) creating and maintaining a positive sense of self is a basic human endeavor (Baumeister, 1998); (b) feeling good about oneself, personal success, and having many unique or distinctive personal attitudes and opinions are valued (Oyserman \& Markus, 1993; Triandis, 1995); and (c) abstract traits (as opposed to social, situational descriptors) are central to self-definition (Fiske, Kitayama, Markus, \& Nisbett, 1998). Second, with regard to well-being, individualism implies that open emotional expression and attainment of one's personal goals are important sources of well-being and life satisfaction (Diener \& Diener, 1995; Markus \& Kitayama, 1991). Third, individualism implies that judgment, reasoning, and causal inference are generally oriented toward the person rather than the situation or social context because the decontextualized self is assumed to be a stable, causal nexus (Choi, Nisbett, \& Norenzayan, 1999; Miller, 1984; Morris \& Peng, 1994; Newman, 1993). Consequently, individualism promotes a decontextualized, as opposed to a situation-specific, reasoning style, one that assumes social information is not bound to social context.

Last, with regard to relationality, individualism implies a somewhat ambivalent stance. Individuals need relationships and group memberships to attain self-relevant goals, but relationships are costly to maintain (Kagitcibasi, 1997; Oyserman, 1993). Theorists assume that individualists apply equity norms to balance relationships' costs and benefits, leaving relationships and groups when the costs of participation exceed the benefits and creating new relationships as personal goals shift. Therefore, theorists assume that for individualists, relationships and group memberships are impermanent and nonintensive (Bellah et al., 1985; U. Kim, 1994; Shweder \& Bourne, 1982).

\section{Collectivism}

The core element of collectivism is the assumption that groups bind and mutually obligate individuals. From this core, theorists discern a number of plausible consequences or implications of collectivism. One question we explore further is whether research has empirically validated these plausible consequences or implications cross-culturally and whether these plausible consequences are, in fact, universal consequences of collectivism.

Although sometimes seen as simple opposites, it is probably more accurate to conceptualize individualism and collectivism as worldviews that differ in the issues they make salient (Kagitcibasi, 1987, 1997; Kwan \& Singelis, 1998). According to Schwartz (1990), collectivist societies are communal societies characterized by diffuse and mutual obligations and expectations based on ascribed statuses. In these societies, social units with common fate, common goals, and common values are centralized; the personal is simply a component of the social, making the in-group the key unit of analysis (e.g., Triandis, 1995). This description focuses on collectivism as a social way of being, oriented toward in-groups and away from out-groups (Oyserman, 1993). Because in-groups can include family, clan, ethnic, religious, or other groups, Hui (1988) and Triandis (1995), among others, have proposed that collectivism is a diverse construct, joining together culturally disparate foci on different kinds and levels of referent groups. In this way, collectivism may refer to a broader range of values, attitudes, and behaviors than individualism.

Plausible consequences of collectivism for psychology - selfconcept, well-being, attribution style, and relationality — are easily discerned. First, with regard to the self, collectivism implies that (a) group membership is a central aspect of identity (Hofstede, 1980; Hsu, 1983; U. Kim, 1994; Markus \& Kitayama, 1991) and (b) valued personal traits reflect the goals of collectivism, such as sacrifice for the common good and maintaining harmonious relationships with close others (Markus \& Kitayama, 1991; Oyserman, 1993; Triandis, 1995). Second, with regard to well-being and emotional expression, collectivism implies that (a) life satisfaction derives from successfully carrying out social roles and obligations and avoiding failures in these domains (U. Kim, 1994; Kwan \& Singelis, 1998; Markus \& Kitayama, 1991) and (b) restraint in emotional expression, rather than open and direct expression of personal feelings, is likely to be valued as a means of ensuring in-group harmony.

Third, with regard to judgment, causal reasoning, and attributions, definitions of collectivism suggest that (a) social context, situational constraints, and social roles figure prominently in person perception and causal reasoning (Miller, 1984; Morris \& Peng, 1994) and (b) meaning is contextualized and memory is likely to contain richly embedded detail. Last, with regard to relationality, definitions of collectivism imply that (a) important group memberships are ascribed and fixed, viewed as "facts of life" to which people must accommodate; (b) boundaries between in-groups and out-groups are stable, relatively impermeable, and important; and (c) in-group exchanges are based on equality or even generosity principles (U. Kim, 1994; Morris \& Leung, 2000; Sayle, 1998; Triandis, 1995).

\section{PSYCHOLOGICAL STUDY AND ASSESSMENT OF INDIVIDUALISM AND COLLECTIVISM}

The previous section highlighted plausible consequences of individualism and collectivism without operationalizing individualism and collectivism. In this section, we provide a guide to the study and assessment of individualism and collectivism based on the past 20 years of usage. Our focus is on literature emerging since 1980 , because references to individualism and collectivism in the psychological literature increased dramatically at about that time and because in 1980 Hofstede published his highly influential analysis of cultural frame, in which individualism was a central focus.

\section{Overview and Background}

As conceptual frameworks, individualism (IND) and collectivism (COL) reflect clearly contrasting worldviews. Our review seeks to shed light on the question, What is the empirical validation of these theoretical frames, especially with regard to the assumption that Americans are a gold standard of high individu- 
alism (and low collectivism)? We attempted to include all relevant studies with American participants published since 1980. For the purpose of the present review, we include as American both Canada and the United States for three reasons. First, the field has clearly assumed an approximate equivalence between the cultures of Canada and the United States in terms of IND and COL. Second, the one empirical study we found examining this assumption does support this conclusion (Kemmelmeier et al., 2001). Third, nearly all of the experimental cross-cultural self-concept research contrasts Canadians and Japanese. So as not to introduce ambiguity, we specify which country our American samples come from both in the appendixes and in the text so the reader can distinguish the source.

\section{Source of Data}

The meta-analyses reported in this article included studies assessing IND and/or COL, whereas the review of psychological implications of IND-COL included all studies in which IND and/or COL were evoked as an explanation for self-concept, well-being, attribution style, or relationality. We also included studies using parallel terms, such as discussions of Western versus Eastern worldviews (e.g., Choi \& Nisbett, 1998; Miller, 1984; Morris, Nisbett, \& Peng, 1995; Morris \& Peng, 1994). To obtain articles, we first searched electronic databases (e.g., PsycINFO, ERIC, and Dissertation Abstracts International) for publications from 1980 through 1999, using the following keywords: individualism, collectivism, independence, interdependence, self-construal, allocentrism, and idiocentrism. To obtain unpublished or in-press research, we used professional e-mail lists. As listed in Appendixes A, B and C, 83 different studies were included in the metaanalyses of IND or COL, and 170 studies were included in the review of psychological implications of IND and COL (studies are listed in each relevant appendix).

\section{Limitations of the Current Data}

Like any review of extant literature, the nature of the current knowledge base limits the conclusions and generalizations one can draw. Although Hofstede (1980) explicitly focused on differences between countries and not individuals, this element of his work has not caught on, and most research studies individual differences. Some authors do focus on analysis of cultural representations such as news reports (e.g., F. Lee, Hallahan, \& Herzog, 1996); however, most current models focus on assessing IND at the individual level (see Triandis, 1995). These levels of analyses are not contradictory, and it is possible in principle to contrast social artifacts, groups, and individuals differing in IND.

The preponderance of individual-level analyses is not surprising. Country-level comparisons require enormous resources because these analyses require the researcher to sample a sufficient number of distinct groups to allow for quantitative analyses. Not only must sufficient groups be sampled, but these groups must also be at least reasonably representative of the society as a whole if one is to generalize comfortably to a society. In contrast to these desired characteristics, we found that cross-cultural analyses typically contrast two groups of students. Researchers most commonly use a single cross-group comparison. Comparisons focus on either showing that variables of interest vary in ways that might be expected if cross-cultural differences in IND or COL exist without directly showing the connection to IND or COL or assessing IND or COL to show that differences in IND or COL predict differences in the variables of interest. Confusion arises in the current literature because authors commonly describe their research in cross-national terms although their data are at the individual level. This use of a single cross-group comparison to generalize about cross-societal differences is a central limitation of the existing database.

Three additional limitations of our database are the narrow focus on undergraduates as research participants, ${ }^{1}$ single group contrasts, and enormous heterogeneity in how researchers conceptualize and operationalize IND and COL. Using undergraduates clearly limits generalizability to other segments of society. Narrow focus on differences between European American undergraduates and undergraduates from either a single East Asian country or a single American racial or ethnic minority group limits generalizability to other countries, racial groups, or ethnic groups. The bulk of crossnational research comes from comparisons of American undergraduates with undergraduates from Japan, Hong Kong, People's Republic of (PR) China, or Korea. The final limitation of our database involves heterogeneity of usage and conceptualization of IND and COL in the literature. This heterogeneity challenged our ability to generalize about IND and COL and required us to establish a working structure to integrate diverse approaches for this review. We outline these usage and conceptualization issues and our working solution in the next section.

\section{Measuring IND and COL}

\section{Hofstede's (1980) Approach}

One approach to operationalizing and measuring IND is to focus on cultural values assessed at the aggregate level, emphasizing difference between cultural units. In his groundbreaking effort, Hofstede (1980) surveyed samples of employees of the same multinational corporation in 39 nations. On the basis of the different samples' responses to a work satisfaction questionnaire, he generated country-level indicators of IND, conceptualizing it as a function of workplace values. Hofstede assumed that IND and COL formed a single continuum, with low IND isomorphic with high COL. Hofstede argued that IND was in part a reflection of social-structural conditions. He demonstrated consistent associations between his aggregate measure of IND and country-level indicators of the society - gross national product (GNP), country latitude, population size, and density.

Hofstede (1980) was careful to point out the limitations of his research. First, he emphasized that his country-level analysis of IND could not explain individual behavior, which he regarded as a theoretically distinct problem. Second, he saw country-level IND as an indicator embedded in a dynamic process of cultural development-shifting as social, structural, and historical changes occur. Thus, Hofstede warned that his own results were not stable but rather shaped by the economic and historical circumstances of the

\footnotetext{
${ }^{1}$ Over $80 \%$ of studies in the meta-analyses used undergraduates (three studies used adults, three used management trainees, and two used managers), as did a similar percentage of studies in the narrative review (7\% of studies used children and $12 \%$ used adults). See Appendixes A-C.
} 
1970s, when he collected his data. He was able to demonstrate this flux by comparing responses collected a few years apart in his first and second data sets. These data demonstrate cultural shift; for example, Japanese workers shifted toward higher IND. Whereas Hofstede's work was highly influential, his focus on social structures and use of a national survey to assign cultural values to a country has not become a common practice (but see Vandello \& Cohen, 1999). Perhaps this is because implementation of this method is time and resource intensive and because attention has shifted to the ways that cultural frames influence individuals.

\section{Current Approaches}

Three general approaches are in current use by researchers studying IND-COL. We termed these approaches applying Hofstede, direct assessment, and priming cultural frame. None of these approaches dominate the field; each has limitations and any decision to limit our review to particular approaches would have dramatically narrowed its scope. For example, omitting research that did not assess IND-COL would have eliminated most U.S.India comparisons and all self-esteem research because researchers in these areas do not assess IND-COL. We outline each approach with its strengths and limitations below.

\section{Applying Hofstede}

Despite Hofstede's (1980) admonitions, a large proportion of research uses his ratings of country-level IND as proxies for IND rather than assessing IND directly. Some researchers use the ratings he provided; others simply note that Hofstede found a difference between two countries and then use this as the basis of their assumption that the two countries still differ in IND and that their findings relate to this difference. Either variant of the applying Hofstede approach makes at least three assumptions about mean levels of IND as assessed by Hofstede: that they are (a) accurate across life domains (e.g., self-concept or well-being), (b) stable over time, and (c) relevant to individual-level assessment. Researchers who use this approach also typically assume European Americans are higher in IND than their comparison group (usually East Asians). Lack of empirical support for these assumptions makes this approach vulnerable to criticism.

\section{IND-COL Rating Scales}

A second common approach is to measure IND and COL at the individual level and to correlate this assessment with individual outcomes, behaviors, attitudes, and beliefs. When using this approach to assess IND-COL, researchers typically ask respondents to rate how much they agree with or how important they find a list of behaviors, attitudes, and value statements. This approach avoids the assumptions required to apply Hofstede; however, it has limitations as well. First, the direct assessment approach assumes that cultural frame is a form of declarative knowledge (e.g., attitudes, values, and beliefs) that respondents can report on rather than some set of more subtle and implicit practices and social structures that respondents cannot report on because these practices are deeply woven into everyday life and are a normal part of living. Second, this approach assumes cross-cultural convergence in the meaning assigned to scale-response choices. Response choices are typically vague quantifiers such as "very much agree" or "very important." Comparing responses to IND or COL scales cross-culturally requires that researchers assume that what respondents mean when they say that they agree is sufficiently similar cross-culturally to make comparisons meaningful. We found two studies (Ji, Schwarz, \& Nisbett, 2000; Peng, Nisbett, \& Wong, 1997) that address this issue, both comparing China and the United States. Both studies suggested that researchers cannot easily assume common understanding of what responses such as "very important" mean and that scale use can systematically differ between countries. ${ }^{2}$ A final limitation of the direct assessment approach is the assumption of cross-cultural convergence in the questions that must be answered to tap into the underlying dimensions of IND and COL. To date, few studies have applied strict psychometric criteria to carefully examine equivalence in cross-cultural measurement (see Bontempo, 1993; Rhee et al., 1996, for exceptions). Results have focused on specific instruments, and as will be seen, a large number of instruments and operationalizations are in current usage.

\section{Priming Studies}

Given the limitations in the applying Hofstede and direct assessment approaches, it is not surprising that researchers have sought alternatives. An emerging alternative, based in social cognition research, involves efforts to prime IND or COL values or independent-interdependent self-definitions before assessing their effect on a dependent measure. Social cognition research consistently shows first that accessible knowledge influences behavior and second that temporarily accessible and chronically salient knowledge produce equivalent effects in laboratory settings (e.g., Bargh, Bond, Lombardi, \& Tota, 1986). These findings form the theoretical underpinning of priming research in cultural psychology. These priming techniques attempt to create an experimental analogue of chronic differences between cultural groups by temporarily focusing participants' attention on different cultural content or values.

There are two types of priming manipulations: The first type aims at making a participant's IND and COL values salient, and the second type aims at making a general IND or COL worldview salient. In its simplest version, cultural values are primed as follows: Experimental participants complete an IND-COL scale immediately prior to responding to the dependent variable, and control participants respond to the dependent variable prior to completing the IND-COL scale (e.g., Oyserman, Sakamoto, \& Lauffer, 1998). This technique allows researchers to compare responses of participants who have just brought to mind their cultural values and beliefs with the responses of participants not focused on their cultural values. Studies of this sort can show the effect of culture by focusing participants' attention on cultural values. This technique allows researchers to study the effect of bringing cultural frame to mind and whether the strength of this

\footnotetext{
${ }^{2}$ Perceived options anchor responses, so even a little individualism within a Chinese context would stand out and be rated more extremely (Peng, Nisbett, \& Wong, 1997). Response scales themselves can influence judgments of Chinese and American respondents differently; scale structure influenced American but not Chinese respondents' judgment of their own public behaviors (Ji, Schwarz, \& Nisbett, 2000).
} 
effect depends on individual endorsement of cultural values. Therefore this priming technique provides greater clarity in causal reasoning than a simple correlational approach, in which researchers correlate an IND-COL scale and a dependent measure, because systematic variation in the strength of the relationship can be attributed to salience of IND-COL. A limitation of this technique is that the IND-COL scales are typically the same kind of direct assessments just critiqued; therefore the previously noted limitations of direct assessment can be said to apply to this type of manipulation as well.

A second type of priming manipulation focuses participants' attention either on IND or on COL and compares their subsequent responses on a dependent measure. In other words, this technique temporarily influences whether one's IND-related or COL-related values, beliefs, and cognitions are more likely to come to mind but does not attempt to measure these values, beliefs, and cognitions directly. In the first published application of this method, Trafimow, Triandis, and Goto (1991) asked participants to describe the ways they were either different from or similar to their family and friends. The first prime elicited individualistic, or independent, self-knowledge, whereas the second elicited collectivistic, or interdependent, self-knowledge. More recently, Gardner and colleagues (Brewer \& Gardner, 1996; Gardner, Gabriel, \& Lee, 1999) primed IND and COL self-knowledge by having participants read a brief paragraph and circle either first person singular or plural pronouns. They found that focusing on pronouns like $I, m e$, and myself made independent self-knowledge more accessible, whereas a focus on pronouns like we, us, and ourselves drew attention to a person's embeddedness in a collective and thus made interdependent self-knowledge more salient. This technique has the advantage of avoiding measurement altogether by experimentally creating IND or COL differences in focus of attention. This technique avoids problems of direct assessment and, unlike the applying Hofstede approach, allows researchers to study culture as a dynamic process. Like other approaches, priming is limited by lack of data comparing results using the different techniques, leading to ambiguity as to the robustness of findings across measurement paradigms.

\section{Summary}

In reviewing the implications of IND-COL for basic psychological domains, we review studies that used any of the approaches outlined above. We chose this broadly inclusive approach for three reasons: (a) Each approach has limitations, (b) none dominates the field, and (c) method and typical country of comparison differed by content domain, therefore picking one method would also result in reducing the scope of our reviews. Where possible, we highlight convergence and divergence in findings based on these approaches.

\section{Operationalizing IND-COL}

Although the assumption of IND being the conceptual opposite of COL may be intuitively appealing, an accumulation of recent research suggests this simple approach does not sufficiently represent the impact of IND and COL on basic psychological processes. Instead, IND and COL are better understood as domainspecific, orthogonal constructs differentially elicited by contextual and social cues (Bontempo, 1993; Kagitcibasi, 1987; Oyserman, 1993; Rhee et al., 1996; Singelis, 1994; Sinha \& Tripathi, 1994; Triandis, Bontempo, Villareal, Asai, \& Lucca, 1988). Following Schwartz (1994), it seems more reasonable to view societies as dealing with collective- and individual-oriented value choices separately. This means any given society is likely to have at least some representation of both individualistic and collectivistic worldviews. However, this formulation leaves open the question of whether in all societies IND (focus on the independent individual) and COL (focus on duty and obligation to in-groups) necessarily carry with them all the related constructs described in the section on implications. Thus, for example, a focus on personal achievement may be multiply determined and not always be related to individualistic values, just as seeking the advice of parents may be multiply determined and not always be related to collectivistic values.

\section{Terminology in Current Use}

It is most common for research to refer to an individual focus as individualism and to a collective focus as collectivism. This simple usage allows much diverse research to be thought of as an integrated whole and is the most commonly accepted way of describing the phenomena. Although this simple usage introduces heterogeneity because it distinguishes neither differences in IND and COL scales nor differences in levels of analyses, we feel that the advantage of continuing to use these general terms outweighs the disadvantages because research primarily refers to the individual level of analyses and because scales contain many common features.

That is not to say that a number of other terms are not applicable. For example, to clarify their focus on the individual level of analyses, Markus and Kitayama (1991) proposed the terms independence and interdependence to describe the self-related aspects of IND and COL. Triandis coined the terms idiocentrism and allocentrism as the individual-level equivalents of country- or society-level IND and COL (Triandis, 1995). Whereas most empirical research is at the individual level, neither of these alternative conceptions has fully captured the literature, and different terminologies persist. For example, researchers describing the effects of situationally primed IND-COL refer to priming individualism-collectivism (Kemmelmeier, Wieczorkowska, Erb, \& Burnstein, in press, Study 3; Oyserman et al., 1998), independence-interdependence (Gardner et al., 1999), idiocentrism-allocentrism, and private self-collective self (Trafimow et al., 1991). To avoid unduly limiting the scope of this research, we include all relevant research, however labeled. We refer to the effects of IND and COL, using the more specific terms as needed to avoid confusion.

\section{Existing IND-COL Scales}

\section{Types of Scales}

Hofstede (1980) assessed only IND because he assumed that COL is equivalent to low IND. This bipolar single dimension approach continues to have some supporters (see Appendixes A-C). When researchers contrast countries on IND-COL, they assess only IND (or COL) in a substantial minority of cases. We 
found this approach in almost a third of the studies in the metaanalyses (26 of 83 studies). If anything, this bipolar single dimension approach is more popular among researchers studying psychological implications of IND-COL. Of the 170 studies examining psychological implications of IND-COL, only 87 assessed IND or COL and only $40 \%$ of these $(n=36)$ assessed both IND and COL.

\section{Measurement Technique}

Triandis and colleagues proposed multimethod assessment as the most valid way to assess the cultural syndromes associated with IND and COL (Triandis, Chan, Bhawuk, Iwao, \& Sinha, 1995; Triandis, McCusker, \& Hui, 1990). However, perhaps because of the effort and difficulty involved in a multimethod assessment, Likert-type ratings of values and attitudes are by far the most prevalent method used to assess IND-COL. Given their dominance, our review focuses on these types of scales. In examining the scales cited in the past 20 years, we did not find a single standard or most common measure, though some items are common across many scales. In an effort to make sense of these measures, we sorted scales into messy categories, finding 27 distinct scales. Categories were messy and necessarily somewhat arbitrary because researchers often modify scales with each use. Distinct scales primarily contained items not previously published in another scale or items from so many previously published scales that no single source was primary. Eleven of these distinct scales measured IND-COL as a single bipolar construct and 16 measured IND (or independence or idiocentrism) and COL (or interdependence or allocentrism) as orthogonal constructs. In some cases, authors refer to subscales of IND-COL. For example, Triandis used more specific terms, such as self-reliance with hedonism, separation from in-groups, and family integrity (Triandis et al., 1986), and self-reliance with competition, distance from in-groups, and concern for in-groups (Triandis et al., 1988).

\section{Content of Current Scales}

Given the diverse terminology used in IND-COL research and the different topics addressed in measurement instruments of INDCOL, we conducted a thorough review and content analysis of scales used in this area to clarify what IND and COL refer to. We content-coded each item on the 27 available IND-COL scales, distinguishing COL-focused and IND-focused items with each item assigned to only one content category (for more information, see Coon \& Oyserman, 2001). All three authors coded most scales jointly using mutually agreed on guidelines, with differences resolved through discussion; two authors coded remaining scales.

As shown in Table 1, we identified seven IND and eight COL components, accounting for $88 \%$ of items across each of the

Table 1

Individualism and Collectivism Domains Assessed in Individualism-Collectivism Scales

\begin{tabular}{|c|c|c|}
\hline Domain name & Description & Sample item \\
\hline \multicolumn{3}{|c|}{ Individualism } \\
\hline Independent & $\begin{array}{l}\text { Freedom, self-sufficiency, and control } \\
\text { over one's life }\end{array}$ & $\begin{array}{l}\text { I tend to do my own thing, and others in } \\
\text { my family do the same. }\end{array}$ \\
\hline Goals & $\begin{array}{l}\text { Striving for one's own goals, desires, } \\
\text { and achievements }\end{array}$ & $\begin{array}{l}\text { I take great pride in accomplishing what } \\
\text { no one else can accomplish. }\end{array}$ \\
\hline Compete & Personal competition and winning & $\begin{array}{l}\text { It is important to me that I perform } \\
\text { better than others on a task. }\end{array}$ \\
\hline Unique & $\begin{array}{l}\text { Focus on one's unique, idiosyncratic } \\
\text { qualities }\end{array}$ & $\begin{array}{l}\text { I am unique-different from others in } \\
\text { many respects. }\end{array}$ \\
\hline Private & Thoughts and actions private from others & I like my privacy. \\
\hline Self-know & $\begin{array}{l}\text { Knowing oneself; having a strong } \\
\text { identity }\end{array}$ & I know my weaknesses and strengths. \\
\hline Direct communicate & $\begin{array}{l}\text { Clearly articulating one's wants and } \\
\text { needs }\end{array}$ & I always state my opinions very clearly. \\
\hline \multicolumn{3}{|c|}{ Collectivism } \\
\hline Related & $\begin{array}{l}\text { Considering close others an integral part } \\
\text { of the self }\end{array}$ & $\begin{array}{l}\text { To understand who I am, you must see } \\
\text { me with members of my group. }\end{array}$ \\
\hline Belong & $\begin{array}{l}\text { Wanting to belong to and enjoy being } \\
\text { part of groups }\end{array}$ & $\begin{array}{l}\text { To me, pleasure is spending time with } \\
\text { others. }\end{array}$ \\
\hline Duty & $\begin{array}{l}\text { The duties and sacrifices being a group } \\
\text { member entails }\end{array}$ & $\begin{array}{l}\text { I would help, within my means, if a } \\
\text { relative were in financial difficulty. }\end{array}$ \\
\hline Harmony & $\begin{array}{l}\text { Concern for group harmony and that } \\
\text { groups get along }\end{array}$ & $\begin{array}{l}\text { I make an effort to avoid disagreements } \\
\text { with my group members. }\end{array}$ \\
\hline Advice & Turning to close others for decision help & $\begin{array}{l}\text { Before making a decision, I always } \\
\text { consult with others. }\end{array}$ \\
\hline Context & $\begin{array}{l}\text { Self changes according to context or } \\
\text { situation }\end{array}$ & $\begin{array}{l}\text { How I behave depends on who I am } \\
\text { with, where I am, or both. }\end{array}$ \\
\hline Hierarchy & Focus on hierarchy and status issues & $\begin{array}{l}\text { I have respect for the authority figures } \\
\text { with whom I interact. }\end{array}$ \\
\hline Group & A preference for group work & $\begin{array}{l}\text { I would rather do a group paper or lab } \\
\text { than do one alone. }\end{array}$ \\
\hline
\end{tabular}


scales. With regard to COL scales, the overwhelming majority included at least one item focused on "sense of duty to group" ( $85 \%$ of scales) and "relatedness to others" (74\% of scales). Further, more than half of the COL scales included at least one item focused on "seeking others' advice" (65\%), "harmony" $(57 \%)$, and "working in groups" (57\%). The less commonly assessed components of COL were "sense of belonging to a group" (39\%), "contextual self" (22\%), and "valuing hierarchy" (17\%). With regard to IND scales, $83 \%$ of scales included at least one item focused on "valuing personal independence." One third or fewer of the scales included any of the remaining theoretically plausible six components of IND: "personal achievement" (33\%), "selfknowledge" (33\%), "uniqueness" (30\%), "privacy" (22\%), "clear communication" (19\%), and "competition" (15\%). Thus, although scales are diverse, there is enough overlap in scale content to warrant use of meta-analytic techniques.

Overall, our content analysis of 27 IND-COL scales allowed us to identify consensual operationalization of IND and COL across researchers. For IND, the core or consensually agreed on element is valuation of personal independence. For COL, the core or consensually agreed on element is sense of obligation and duty to the in-group. However, scales vary widely in what other content components they regard as relevant to the measurement of IND and COL. For example, some IND scales include personal uniqueness, valuing privacy, or self-knowledge. Family focus (familialism) is sometimes included in COL, sometimes not, and the same is true of respect for hierarchy and competition, which are sometimes included as part of IND-COL.

Authors disagree as to whether familialism - defined as relatedness to family, seeking harmony with family members, or supporting and seeking advice from family-is separate from COL (Gaines et al., 1997), the essential core of COL (Lay et al., 1998), or an important element of COL distinct from a non-kin-focused type of COL (Rhee et al., 1996). As for hierarchy and competition, Hofstede (1980) originally proposed that IND and "power distance" are separate cultural factors, a view paralleled in Fiske's (1992) taxonomy of basic social relationships and more recently advocated by Triandis, who proposed including hierarchical or egalitarian aspects of social relationships in analyses of IND-COL (cf. Singelis, Triandis, Bhawuk, \& Gelfand, 1995; Triandis \& Gelfand, 1998). By including a horizontal-vertical dimension to discussion of cultural differences, one can distinguish different dimensions of IND and COL depending on whether they presume equal or different status between individuals, namely horizontal IND, horizontal COL, vertical IND, and vertical COL. According to this framework, cultures high in horizontal IND tend to be egalitarian, with individuals being independent and of comparable power and status, whereas cultures high in vertical IND tend to champion competition between individuals, resulting in acceptable inequality between individuals.

Given that there is no consensus in the literature on the roles of family and hierarchy within the IND-COL framework, we paid special attention to findings concerning these issues. Wherever possible, we traced the extent that familialism functioned independently of COL. In addition, we attempted to assess whether hierarchy and personal competition functioned independently of IND and COL. Given the heterogeneity of measurement more generally, we attempted to examine whether differences between countries (or groups) depended on how IND and COL were assessed.

\section{META-ANALYSES OF THE LITERATURE COMPARING (EUROPEAN) AMERICANS WITH OTHERS ON IND-COL}

In this section, we present two sets of meta-analyses focused on difference in level of IND and COL, including both international comparisons and within-U.S. comparisons. Note that the metaanalyses do not address the implications of IND and COL for basic psychological processes; we discuss implications later in the review.

\section{Overview}

Recall that a basic aim of the present review is to answer this question: Are European Americans higher in IND (lower in COL) than other cultural groups? To begin to answer this question, we conducted two groups of meta-analyses. The first group involved a set of international comparisons, contrasting Americans (and Canadians) with people from other countries. The second group involved a set of within-U.S. comparisons, contrasting European Americans and Americans from other ethnic or racial groups. (We found too few studies using Canadians to warrant separate analyses.) For each set, we conducted one analysis for IND scale scores and a separate analysis for COL scale scores, resulting in four sets of meta-analyses. These compared (a) Americans (or Canadians) with people from other countries on measures of IND, (b) Americans (or Canadians) with people from other countries on measures of COL, (c) European Americans with other American ethnic and racial groups on measures of IND, and (d) European Americans with other American ethnic and racial groups on measures of COL.

For international meta-analyses, we aggregated both by region and by country. For within-U.S. meta-analyses, we aggregated both across ethnic groups and by ethnic groups. For both international and within-U.S. meta-analyses, we examined main and moderator effects and analyzed the simultaneous effects of IND and COL by plotting IND-COL effect sizes against each other. Main effects tell us about the size and direction of differences in IND and COL. Moderator effects tell us to what extent scale reliability, scale content, and sample composition (i.e., student vs. nonstudent) influence size and direction of main effect differences. Analyses of simultaneous effects of IND and COL can tell us whether European Americans are indeed both higher in IND and lower in COL than are others.

Eighty-three studies contributed to the four types of comparisons described above. Fifty of these studies provided data for the international comparisons, 35 provided data for the within-U.S. comparisons. Of these studies, 2 provided data relevant to both sets of comparisons (Matsumoto, Weissman, Preston, Brown, \& Kupperbusch, 1997; Rhee et al., 1996), resulting in the total, 83 different studies. Most studies reporting international comparisons omitted description of the ethnic or racial makeup of American undergraduate samples. We assumed these were predominantly European American samples given the ethnic composition of undergraduate students at the universities represented (the same was true for studies using Canadian samples). In our within-U.S. samples we were limited to comparisons among European Americans and African Americans, Asian Americans, and Latino Americans because these were the only groups studied in the literature. This means, for example, that we found no literature comparing European Americans with Native Americans on IND and COL. 
Overview of Computation and Analysis

\section{Computing Effect Sizes}

\section{Main Effects}

The effect size calculated was $g$, the mean difference between samples on IND or COL divided by the pooled standard deviation. In international comparisons, $g$ represents the mean difference between an American sample and a sample from another country. For within-U.S. comparisons, $g$ represents the mean difference between a European American sample and a sample from a specific other American group (e.g., African Americans). Because some samples were small, we applied the sample size correction suggested by Hedges and Olkin (1985). Thus, the final statistic reported is the corrected difference score $d$ rather than $g$.

Most commonly, researchers reported a single mean IND score when assessing IND and a single mean COL score when assessing COL. We computed a single mean score across multiple subscales in the few studies that used subscales to assess IND or COL when the scale's original author recommended this approach (e.g., Hui, 1988). This allowed a straightforward computation of an effect size for two samples. However, in a number of cases, we could not follow this approach either because the original author of a scale did not recommend combining subscales into a single mean score or because the author used multiple measures of IND or COL.

In these latter cases, we first computed effect sizes for each subscale separately and then used a weighting procedure by Gleser and Olkin (1994) to integrate them into a single effect size. If the information concerning the correlation between subscales required by this procedure was not available, we simply averaged effect sizes for all subscales or measures of the same construct. All relevant subscales or measures were included in this procedure, with only one exception. Triandis's most recent IND-COL scale (Singelis et al., 1995; Triandis \& Gelfand, 1998) assesses both horizontal and vertical IND and COL. The two COL subscales were correlated; hence, they were combined into one COL score. However, Triandis designed the vertical and horizontal IND scales to be orthogonal, raising questions as to whether we could treat combined group differences on the two scales as reflections of the same underlying concept. Further, the vertical IND subscale focused exclusively on competition, content that is atypical for all of the other IND scales we found. Therefore, we selected only the horizontal, not the vertical, IND subscale for the six international comparison studies and the nine within-United States studies that used both IND subscales.

We relied on the recommendations of Cohen (1988) in interpreting the meaning of the observed effect sizes - effect sizes $(d)$ of less than 0.4 are described as "small," those from 0.4 to 0.7 as "moderate," and those above 0.7 as "large." For ease of interpretation, we set comparisons such that positive effect sizes reflect higher (European) American IND and COL and negative effect sizes reflect lower (European) American IND and COL. The expected pattern is then positive values for IND effect sizes and negative ones for COL effect sizes.

Overall, data quality was excellent, with little information missing. When published studies did not report necessary information, we contacted authors if the publication date was not more than 6 years earlier. Otherwise, we estimated effect sizes on the basis of the statistical information available, usually with the help of a pertinent computer program (B. T. Johnson, 1993). With this method, there were only two cases in which the available information did not allow us to obtain the standard deviations of the means necessary for the computation of $g$. In these cases, we estimated this information on the basis of studies with similar measures and samples from the same country (Gire \& Carment, 1993; Leung \& Iwawaki, 1988). Examination of studies with complete data revealed heterogeneity of variance was rarely a problem; therefore, when studies used factor analysis and reported standardized factor scores without corresponding standard deviations (Kashima et al., 1995; Triandis et al., 1993), we assumed a standard deviation equal to one and homogeneity of variance across samples within studies to generate standard deviation estimates. We lacked statistical information in only one case-a nonsignificant subscale effect (Leung \& Iwawaki, 1988), for which we assigned an effect size of zero. Using these methods, we were able to compute effect sizes on the basis of available mean scores and standard deviations in all but $2 \%$ of studies; in these latter cases, we computed effect sizes on the basis of $F$ statistics.

\section{Adjustment for Correlated Effect Sizes}

As can be seen in Appendixes A and B, and as detailed in subsequent sections, not all research involved simple cross-cultural comparisons of a sample of European Americans with a sample from a single other country or group. Some researchers compared the same sample of European Americans with a number of other country or group samples. For example, the researcher could compare the same sample of European Americans with both a sample of African Americans and a sample of Hispanic Americans or the same sample of Americans with both a sample from Japan and a sample from Hong Kong. Because in these cases the researcher used the same sample of Americans to compute effect sizes for more than one comparison (e.g., the U.S.-Japanese comparison and the U.S.-Hong Kong comparison), the resulting effect sizes are stochastically nonindependent. The presence of stochastic dependence violates the assumed independence of observations in conventional meta-analysis methods (e.g., Hedges \& Olkin, 1985; Hunter, Schmidt, \& Jackson, 1982) and results in misspecification of the standard error of the combined effect size estimates and a distortion of Type I error (Becker, 2000; Gleser \& Olkin, 1994; cf. Raudenbush, Becker, \& Kalaian, 1988).

Becker (2000) suggested a number of different strategies to deal with nonindependent effect sizes. The most preferable strategy, to statistically model this nonindependence in the data, requires that the researcher have a database that allows for reliable estimation of the degree of association between pairs of effect sizes. That is, to be able to model nonindependence, the researcher needs a database that includes a sufficient number of studies that make exactly the same multiple comparisons. For example, multiple studies comparing a U.S. sample to both a sample from Japan and a sample from Hong Kong would allow the reliable estimation of the U.S.Japanese effect size and the U.S.-Hong Kong effect size. We found multiple studies with the same multiple comparisons to statistically model nonindependence in the within-U.S. analyses but not in the international analyses.

Specifically, for within-U.S. comparisons we used a generalized least squares estimation method that allows for statistically correct 
estimation of combined effect sizes and their variance (by taking their covariance with other effect sizes into account; see Gleser \& Olkin, 1994 for computational details). This state-of-the-art generalized least squares method of dealing with stochastically dependent effect sizes (Becker, 2000) provides combined effect size coefficient estimates, $\beta_{\mathrm{I}}$, and confidence intervals, $\beta_{\mathrm{i}} \pm z_{\alpha / 2}$ (var $\left.\left(\beta_{\mathrm{i}}\right)\right)^{1 / 2}$, that are similar in interpretation to $d_{\mathrm{i}}$ and its confidence interval. An additional benefit of this method is that it allows for comparison of effect sizes. This means that the interested reader can easily compare size of effects for within-U.S. comparisons.

Because our international comparisons did not provide sufficient data to apply this state-of-the-art method, we used the next best method, forming subsets of country comparisons for all countries for which more than one data point was available (cf. Becker, 2000). In this way, we synthesized effect sizes pertaining to each country comparison (e.g., U.S.-Japan) separately, using the univariate approach to meta-analysis proposed by Hedges and Olkin (1985). Because we could not use the least squares estimation method, there was no straightforward way to compare effect sizes for the international comparisons.

\section{Computation of Homogeneity Coefficients}

As suggested by Hedges and Olkin (1985), we generated the homogeneity coefficient $Q$ for both international and within-U.S. analyses. This coefficient shows whether the estimated effect size can be used as a population estimate. In our case, it provided a sense of whether we could generalize from the obtained effect size of difference between European Americans and each group to the expected effect size in the population between European Americans and each group. When $Q$ coefficients are nonsignificant, samples from the same source show consistent differences with the comparison group, meaning that it is possible to generalize from the samples to the population. When $Q$ coefficients are significant, samples drawn from the same source (e.g., region, country, or ethnic group) differ systematically, meaning it is not possible to generalize to the population. Moderator analyses are particularly promising when $Q$ is significant because they may reveal systematic variability in the effect sizes, explaining the within-class heterogeneity. For example, when researchers studying the same two countries compare them on scales that differ in reliability, scale reliability may moderate or systematically influence the extent that the countries appear different from each other-the effect size of the comparison.

\section{Moderators}

We analyzed three possible moderators: scale reliability, scale content, and sample composition.

\section{Scale Reliability}

Whenever possible, we obtained Cronbach's alpha for each measure of IND and COL from each study used. If a single study presented more than one reliability coefficient, we averaged them. We did not attempt to impute reliability for studies for which we could not obtain information, because in our sample of studies, reliability was not consistent, even across studies that used the same measure. We classified as high reliability scales those scales with $\alpha \geq .70$ and as low reliability scales those scales with $\alpha<.70$ (e.g., Stangor, 1998). We assumed high reliability for scales created through factor analyses unless the study provided information that this was not the case.

\section{Scale Content}

As described previously in the section on content analyses and summarized in Table 1, we coded each scale for content. For each scale, we coded for presence-absence of seven components of IND and eight components of COL. This allowed us to contrast effect sizes from studies differing in scale content.

\section{Sample Composition}

As noted in the section on limitations of the database, almost all studies used students as research participants. However, some studies used nonstudent adults. Therefore, we coded for presence of students versus nonstudent adults in the samples. This allowed us to contrast effect sizes from studies differing in their sample composition. Comparisons were possible only for Japan versus America on IND, PR China versus America on COL, and withinU.S. IND and COL comparisons.

\section{International Comparisons}

\section{Overview}

In all, we found 50 studies (reported in 48 research reports or articles) with cross-cultural comparisons involving Americans since $19800^{3}$ Appendix A summarizes each study used in the international meta-analyses. Studies are organized alphabetically by author, and information is provided about year of publication, which countries were compared with the United States, the number of participants in each sample, the percentage of women in the sample, sample composition (e.g., college students, managers), the IND/COL measurement used, effect size, and reliability codes for moderator analyses.

Twenty-seven studies assessed IND and COL, 2 studies assessed IND only, and 21 studies assessed COL only. Thus, 29 studies provided IND data and 48 studies provided COL data. Participants were mostly undergraduates, notable exceptions being studies by Brett and Okumura (1998); C. C. Chen, Meindl, and Hui (1998); Earley (1989, 1993, 1994); Robert (1998); and Yang (1996), who mostly used employees or managers as participants. Most studies were cross-national, comparing IND and/or COL in the United States and one other country. Multinational studies contributed more than one data point to the meta-analyses. These studies compared Americans with 2 (Earley, 1993, 1994), 3 (Chew, 1996; Gudykunst, Matsumoto, Ting-Toomey, Nishida, Kim, \& Heyman, 1996; M. Kim, Hunter, Miyahara, \& Horvath, 1996; Matsumoto et al., 1997; Robert, 1998), 4 (Kashima et al., 1995), 6 (Kemmelmeier et al., 2001), 9 (Triandis et al., 1993), or 38 other countries (Oishi, 2000). In this way, the 29 IND studies provided 90 data points, and the 48 COL studies provided 109 data points.

\footnotetext{
${ }^{3}$ We could not include studies that lacked specific sample information (e.g., Schwartz, 1994; Triandis et al., 1986, 1988).
} 
Individualism

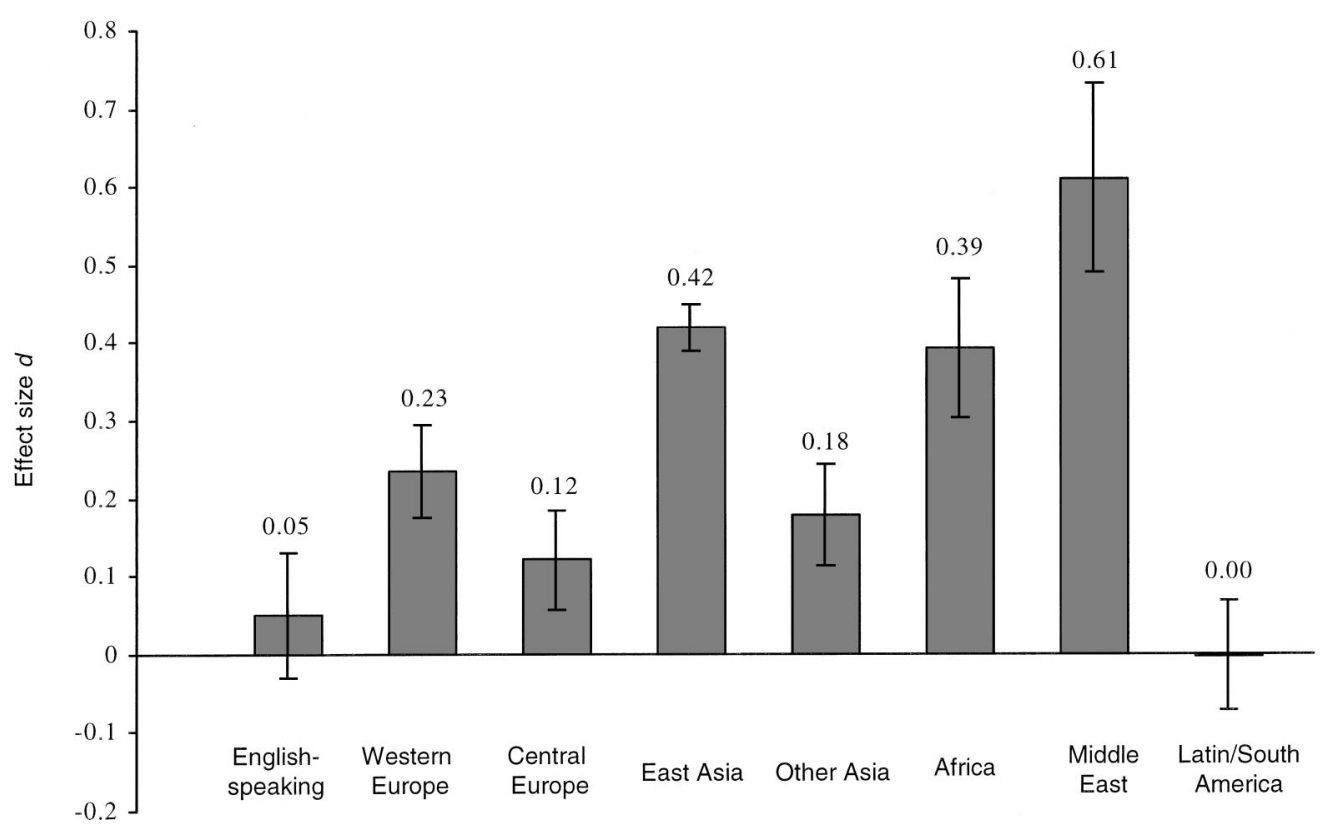

Figure 1. International meta-analysis on individualism: Comparing samples from America and eight regions.

A large number of countries were included in these crossnational comparisons: 46 countries for IND comparisons, 50 countries for COL comparisons. However, few countries provided more than a single comparison, and we obtained two or more IND comparisons for 12 countries and two or more COL comparisons for 14 countries. Most of these more frequently studied countries were South Asian or East Asian (8 of 12 for IND, and 7 of 14 for COL).

\section{Analyses Plan}

We first present analyses describing differences between Americans and others in IND and then analyses describing differences between Americans and others in COL. For each set of analyses we use the following order: (a) regional comparisons, (b) countrylevel comparisons, and (c) moderator analyses (scale reliability, scale content, and sample composition).

\section{Regional-Level Analyses}

We grouped countries into eight regional blocks to contrast regions of the world with Americans (and Canadians). Regional analysis allowed us to integrate many comparisons into a single effect size. The regions we used were (a) English-speaking countries with an early British settlement (White New Zealanders, Australians, South Africans), (b) Western Europe (France, Italy, Spain), (c) Central Europe (Bulgaria, Greece, Poland), (d) East Asia (Japan, PR China, Vietnam), (e) other Asian countries (India, Nepal, Pakistan), (f) Central Africa (Nigeria, Zimbabwe), (g) Middle East (Egypt, Turkey), and (h) Latin/South America (Brazil, Mexico, Puerto Rico). ${ }^{4}$

\section{Country-Level Analyses}

Regional analyses ignore existing differences between countries of the same region and increase the risk of regional overgeneralization (cf. Dien, 1999). Therefore, we also performed a series of country-level analyses for a more appropriate examination of cross-national differences. This method has the advantage of increased specificity and allows us to explore the nature of withinregion heterogeneity. We computed effect sizes for all individual country comparisons and present them graphically. These displays provide the reader with a visual overview of the stability of IND and COL differences across all countries.

\section{Results of International Meta-Analyses on IND}

\section{Regional Analyses}

Figure 1 presents effect sizes comparing samples from America with those from eight world regions. In line with the assumption of an overarching "Western" culture, we found no difference between America and other English-speaking countries and only relatively small differences between Europe (Western and Central) and America, with Americans being higher in IND. The majority of available studies focused on comparisons between East Asians and Americans, making them the most common comparison in the

\footnotetext{
${ }^{4}$ We note two exceptions: (a) Israel was excluded from regional analyses - it did not make sense to include it in the Middle Eastern region, nor could we determine whether it best fit in another region (e.g., Western or Eastern Europe) - and (b) we included Puerto Rico as part of the Latin American (not the U.S.) region.
} 

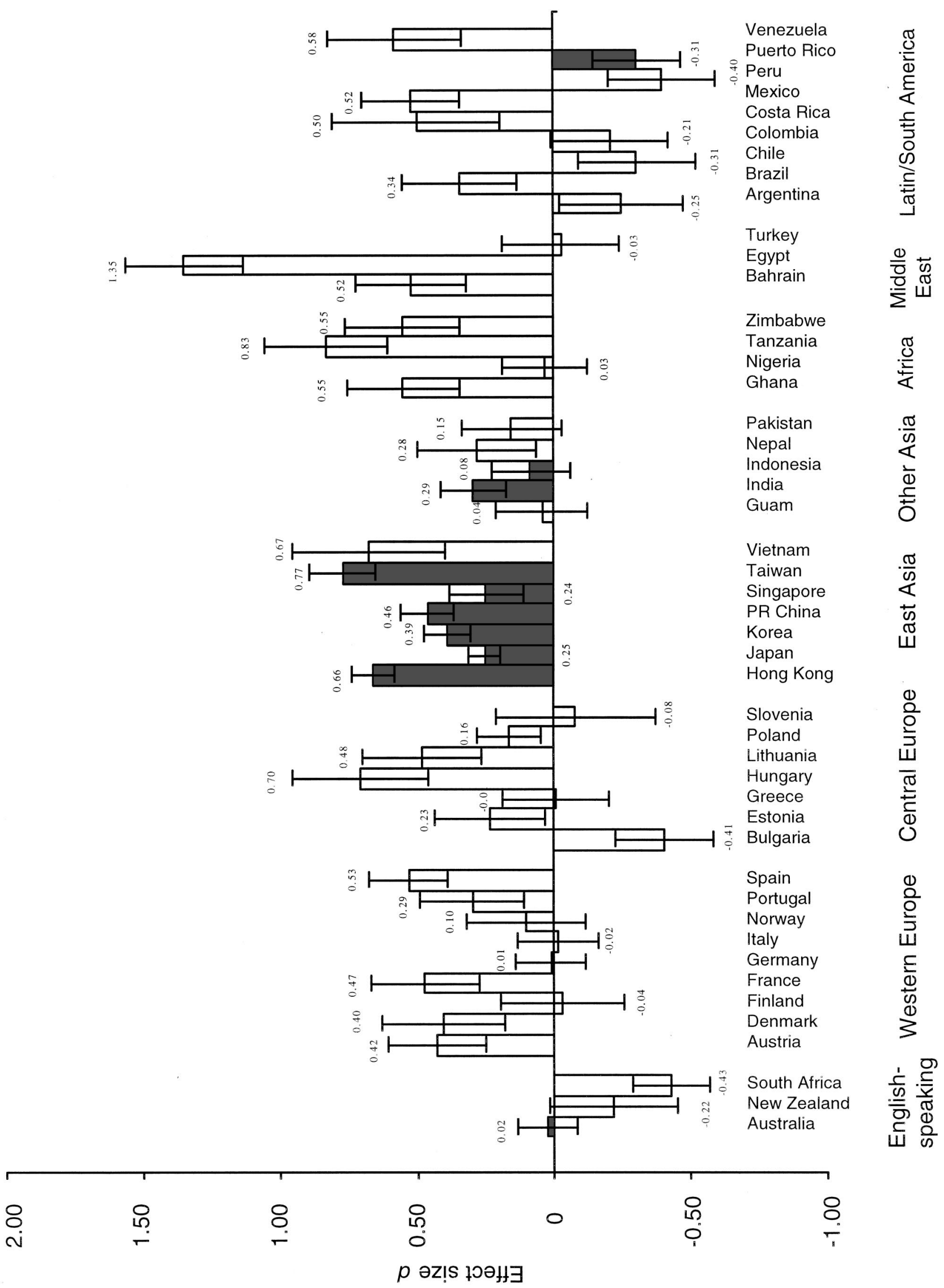
cross-national IND literature. Combined effect sizes for comparisons with East Asia were moderate in size, as were combined effect sizes for Africa and the Middle East. It is surprising that, whereas these findings corroborate conventional expectations of cultural theorists, the combined effect size for "other Asia" was small, and we found no difference in IND between the United States and South America. Recall however that student samples dominated our data set. Because students tend to be higher in socioeconomic status than nonstudents and higher socioeconomic status is associated with higher IND and lower COL (Freeman, 1997; Hofstede, 1980; Triandis et al., 1990), it is possible that these results underestimate regional IND-COL differences among nonstudents.

Nonetheless, regional analyses generally support the assumption that Americans are more individualistic than others are. We turn to more fine-grained analyses of country-level effects as each regional comparison yielded a highly significant $Q$ coefficient (all values of $p<.004$ ), meaning that regional effect estimates do not generalize to the population at large.

\section{Country-Level Analyses}

Figure 2 shows how each country in each region compared with America, highlighting sources of within-region heterogeneity. As seen earlier, for the most part, Americans were more individualistic than were others. Exceptions to this general pattern come from single studies comparing America with a country located in either an English-speaking or a European region (South Africa, New Zealand, Finland, Italy, or Bulgaria). Perhaps more surprising is the observation that Americans were less individualistic than were Latin Americans in more than half of the country samples. Unfortunately, we could not assess the stability of these differences because, except for comparisons with Puerto Rico, these exceptions occurred for countries represented by only one sample in our database.

The meta-analysis allowed examination of the stability of differences between European Americans and others in a subset of comparisons based on more than one sample; this subset of multisample countries included Puerto Rico and some European and Asian countries. As seen in Table 2, European Americans were significantly more individualistic than Hong Kong, Indian, Japanese, Korean, Polish, Singaporean, and Taiwanese respondents. However, they also reported significantly lower IND than Puerto Rican respondents and were not reliably different in IND from Australian, German, or Indonesian respondents. Furthermore, although Americans were more individualistic than were East Asians, effects for comparisons with India, Japan, and Singapore were small. Given nonsignificant $Q$ scores in Indonesia, Poland, Puerto Rico, and Singapore, current evidence is sufficient to conclude that there are no differences in IND between Americans and
Table 2

International Meta-Analyses: Overall Individualism Results Compared With the United States and Canada

\begin{tabular}{|c|c|c|c|c|}
\hline Country & $n$ & $\begin{array}{l}\text { Mean weighted } \\
\text { effect size } \\
\left(d_{i+}\right)\end{array}$ & $95 \% \mathrm{CI}$ & $\begin{array}{l}\text { Homogeneity } \\
\text { within } \\
\text { country }\left(Q_{\text {wi }}\right)\end{array}$ \\
\hline Australia & 3 & 0.02 & $-0.09 / 0.13$ & $6.32 *$ \\
\hline Germany & 3 & 0.01 & $-0.12 / 0.14$ & $51.29 * * *$ \\
\hline Hong Kong & 8 & 0.66 & $0.58 / 0.74$ & $35.10 * * *$ \\
\hline India & 3 & 0.29 & $0.17 / 0.42$ & $40.64 * * *$ \\
\hline Indonesia & 2 & 0.08 & $-0.06 / 0.23$ & 0.32 \\
\hline Japan & 15 & 0.25 & $0.18 / 0.31$ & $549.01 * * *$ \\
\hline Korea & 5 & 0.39 & $0.31 / 0.48$ & $75.01 * * *$ \\
\hline $\begin{array}{l}\text { People's Republic } \\
\text { of China }\end{array}$ & 4 & 0.46 & $0.36 / 0.55$ & $57.07 * * *$ \\
\hline Poland & 3 & 0.16 & $0.05 / 0.28$ & 3.20 \\
\hline Puerto Rico & 2 & -0.31 & $-0.47 /-0.16$ & 0.13 \\
\hline Singapore & 2 & 0.24 & $0.11 / 0.38$ & 1.62 \\
\hline Taiwan & 2 & 0.77 & $0.65 / 0.89$ & $6.72 * *$ \\
\hline
\end{tabular}

Note. Positive values indicate higher American individualism; negative values indicate lower American individualism. Combined effect sizes that differ reliably from 0 are bold. $\mathrm{CI}=$ confidence interval.

$* p<.05$. ** $p<.01$. *** $p<.001$.

Indonesians and that the U.S.-Singapore and U.S.-Poland difference in IND is small. Moreover, the small difference in IND between Puerto Rico and the United States suggests that Puerto Ricans are more individualistic than European Americans. Significant homogeneity coefficients in other country comparisons imply significant variation in effect size among studies with the same country comparison, limiting generalizability. We therefore turn to moderator analyses to explore the impact of differences in scales and other factors influencing effect sizes.

Scale reliability moderator analyses. Our first set of moderator analyses focused on the possibility that effect sizes vary systematically because of differences in scale reliability. Psychometric theory leads to the assumption that if validity is held constant, more reliable scales will show uniformly higher effect sizes than less reliable scales (e.g., Nunnally, 1994). To examine this possibility, we focused on country comparisons in which we had at least two studies with high reliability and at least two studies with low reliability, the minimum number required for this type of analysis. Therefore, country comparisons that provided fewer than four data points overall and country comparisons with fewer than two data points in the high- or low-reliability categories specifically could not be included in the moderator analyses.

These statistical requirements limited our moderator analyses to three countries: Hong Kong, Japan, and Korea. For these countries, results did not follow the simple rule of greater effect size being associated with higher reliability. As can be seen in the first

Figure 2 (opposite). International meta-analysis on individualism: Comparing samples from America and 47 countries. Country comparisons are grouped by region, with countries appearing alphabetically within region. Light bars show effect sizes based on a single data point (a single-sample comparison), and dark bars show effect sizes based on multiple data points. These latter bars represent the countries for which a statistical integration of the available effect sizes is provided and, where possible, moderator analyses were performed. PR China $=$ People's Republic of China. 
Table 3

International Meta-Analyses: Individualism Scale Reliability Moderator Analyses

\begin{tabular}{cccccc}
\hline Country & $\begin{array}{c}\text { Between-class } \\
\text { effect }\left(Q_{\mathrm{B}}\right)\end{array}$ & $n$ & $\begin{array}{c}\text { Mean weighted } \\
\text { effect size }\left(d_{i+}\right)\end{array}$ & $95 \% \mathrm{CI}$ & $\begin{array}{c}\text { Homogeneity within } \\
\text { class }\left(Q_{\mathrm{wi}}\right)\end{array}$ \\
\hline Hong Kong & 0.27 & & & & \\
$\quad$ Low reliability & & 2 & $\mathbf{0 . 6 5}$ & $0.51 / 0.79$ & $12.60^{* * * *}$ \\
$\quad$ High reliability & & 4 & $\mathbf{0 . 6 9}$ & $0.59 / 0.80$ & $16.88^{* * *}$ \\
Japan & $39.01^{* * *}$ & & & & \\
$\quad$ Low reliability & & 9 & 0.08 & $-0.001 / 0.16$ & $371.34^{* * *}$ \\
$\quad$ High reliability & $14.65^{*}$ & 6 & $\mathbf{0 . 4 8}$ & $0.38 / 0.57$ & $138.66^{* * *}$ \\
Korea & & & & \\
Low reliability & & 2 & $\mathbf{0 . 5 5}$ & $0.43 / 0.66$ & $3.18 \dagger$ \\
High reliability & & 3 & $\mathbf{0 . 2 2}$ & $0.10 / 0.34$ & $57.18^{* * *}$ \\
\hline
\end{tabular}

Note. Positive values indicate higher American individualism; negative values indicate lower American individualism. Studies with Cronbach's $\alpha<.70$ were classified as low reliability; studies with Cronbach's $\alpha \geq$ .70 were classified as high reliability. Combined effect sizes that differ reliably from 0 are bold. CI $=$ confidence interval.

$\dagger p<.10$ (marginally significant). $* p<.05$. *** $p<.001$.

column of Table 3, the between-class effect was not significant for Hong Kong, was significant in the expected direction for Japan, and was significant in the opposite direction for Korea. For Hong Kong, effect sizes were not dependent on scale reliability. For Japan, reliable scales were associated with moderate effect sizes, and less reliable scales were associated with nonsignificant differences in IND between Japan and America. Finally, for Korea, effect sizes were smaller for scales that are more reliable and larger for less reliable scales. Given these diverse findings, it is unlikely that reliability alone is responsible for variation in effect sizes. At least in the Korean case, our findings point to a problem of validity, perhaps related to scale content, as explored below.

Scale content moderator analyses. The next set of moderator analyses examined whether observed effect sizes differed as a function of scale content. We examined the moderating role of scale content for all country comparisons for which there were at least two studies that included specific content in their assessment of IND and at least two studies that did not include the same content in their assessment of IND. As with the scale reliability analyses, this criterion excluded country comparisons with fewer than two data points in a given content category. Thus, as detailed below, although there were five studies with Korea comparisons, we were unable to complete a moderator analysis of scale content. First, all five Korea comparisons assessed IND with two of the same IND components, valuing personal independence and uniqueness; hence, we could not compare studies that assessed IND with and without these components. Second, each of the other components of IND appeared only in a single study. Because comparisons require at least two studies with a given content component and two studies without the same content component, we could not make these other comparisons. Similarly, for one of the four studies involving PR China we were unable to obtain any information about the content of the measure used, leaving only three studies, too few to compute moderator analyses.

As reflected in Table 4, sufficient observations were available only for Hong Kong and Japan. In these studies, all IND scales included a common component, valuing personal independence, making it a constant in this analysis. Therefore, analyses of the effect of other components necessarily included the effect of valuing independence. Additional components assessed in either Japan or Hong Kong were valuing personal uniqueness, privacy, direct communication, and self-knowledge (see Table 1 for a description of each domain).

For America-Hong Kong comparisons, scale content did not affect the effect size. Americans were higher in IND no matter whether assessment of IND included uniqueness, privacy, or direct communication, suggesting that the difference between these two countries in IND does not reside in any of these but rather in valuing personal independence. For America-Japan comparisons, scale content was critical for both the size and the direction of effects. Specifically, Americans were higher in IND than were Japanese when (in addition to personal independence) IND assessment included personal uniqueness, valuing privacy, and direct communication. In fact, when IND assessment did not include personal uniqueness, Americans were lower in IND than were Japanese. Further, the effect size jumped from small to large when valuing personal privacy was included (with a similar but less dramatic increase when direct communication was included). These data are suggestive of the notion that the between U.S.Japan difference in IND resides in a combination of different valuation of independence, personal uniqueness, personal privacy, and direct communication.

In contrast, when competition was included in the scale, the difference between Americans and Japanese in IND disappeared, suggesting that competitiveness is a construct unrelated to IND. This conclusion finds support in Triandis's (1995; Singelis et al., 1995) advocacy of assessing competition as a cultural factor separate from IND. Further, the self-knowledge component did not have any influence on effect size, suggesting it is not relevant to the assessment of IND with respect to differences between Japan and America. These findings are interesting, and for selfknowledge provocative, because they seem to contradict basic assumptions about the influence of IND on American and Japanese self-concepts. However, caution in generalizing is necessary because, as can be seen in the last column of Table 6 , the combined effect sizes are quite heterogeneous. Moreover, some of the effects may be due to the specific scale used rather than the content domains more generally-privacy and self-knowledge each ap- 
Table 4

International Meta-Analyses: Individualism Scale Content Moderator Analyses

\begin{tabular}{|c|c|c|c|c|c|}
\hline Country & $\begin{array}{l}\text { Between-class } \\
\text { effect }\left(Q_{\mathrm{B}}\right)\end{array}$ & $n$ & $\begin{array}{l}\text { Mean weighted } \\
\text { effect size }\left(d_{i+}\right)\end{array}$ & $95 \% \mathrm{CI}$ & $\begin{array}{l}\text { Homogeneity within } \\
\text { class }\left(Q_{\mathrm{wi}}\right)\end{array}$ \\
\hline \multicolumn{6}{|c|}{ Hong Kong (independent) } \\
\hline Unique & 0.07 & & & & \\
\hline Included & & 6 & 0.66 & $0.57 / 0.75$ & $28.56 * * *$ \\
\hline Not included & & 2 & 0.68 & $0.51 / 0.86$ & $6.46 *$ \\
\hline Private & 2.04 & & & & \\
\hline Included & & 2 & 0.76 & $0.60 / 0.92$ & $8.97 * *$ \\
\hline Not included & & 6 & 0.63 & $0.53 / 0.72$ & $24.09 * * *$ \\
\hline Direct communicate & 0.48 & & & & \\
\hline Included & & 5 & 0.65 & $0.55 / 0.74$ & $27.23 * * *$ \\
\hline Not included & & 3 & 0.71 & $0.55 / 0.87$ & $7.39 *$ \\
\hline \multicolumn{6}{|l|}{ Japan (independent) } \\
\hline Competition & $5.37 *$ & & & & \\
\hline Included & & 2 & -0.01 & $-0.24 / 0.22$ & 0.39 \\
\hline Not included & & 13 & 0.27 & $0.20 / 0.33$ & $543.25 * * *$ \\
\hline Unique & $216.80 * * *$ & & & & \\
\hline Included & & 11 & 0.49 & $0.42 / 0.55$ & $140.80 * * *$ \\
\hline Not included & & 4 & -0.66 & $-0.79 /-0.52$ & $191.42 * *$ \\
\hline Private & $70.28 * * *$ & & & & \\
\hline Included & & 2 & 0.82 & $0.67 / 0.96$ & 1.44 \\
\hline Not included & & 13 & 0.12 & $0.06 / 0.19$ & $477.30 * * *$ \\
\hline Direct communicate & $16.45 * * *$ & & & & \\
\hline Included & & 6 & 0.39 & $0.30 / 0.48$ & $69.07 * * *$ \\
\hline Not included & & 9 & 0.13 & $0.05 / 0.22$ & $463.49 * * *$ \\
\hline Self-know & 0.32 & & & & \\
\hline Included & & 2 & 0.29 & $0.12 / 0.45$ & $22.85 * * *$ \\
\hline Not included & & 13 & 0.24 & $0.17 / 0.31$ & $525.84 * * *$ \\
\hline
\end{tabular}

Note. Positive values indicate higher American individualism; negative values indicate lower American individualism. In parentheses after the country name is the content contained in all or all but one research instrument used in studies contributing to the country comparison. Studies for which no reliability information was available were excluded. Combined effect sizes that differ reliably from 0 are bold. CI $=$ confidence interval.

$* p<.05$. ** $p<.01$. *** $p<.001$.

peared in only one scale (Gudykunst et al., 1996, for privacy, and Singelis et al., 1995, for self-knowledge), which means that idiosyncrasies of these scales cannot be disentangled from the effect of scale content. Overall, Americans were higher in IND than East Asians were. However, effects varied from sample to sample, and scale reliability or content did not fully account for this variability.

Sample composition moderator analyses. Following the criteria of needing at least four studies in a country comparison for a moderator analysis (in this case, two using students and two using nonstudent adults in their samples) we were able to analyze the possibility of moderation due to sample composition for the Japan-America comparison only. Two of these 15 studies sampled nonstudent adults. We found that with nonstudent adults, the effect size was somewhat larger $(d=0.44$, confidence interval [CI]: 0.20 to 0.68$)$ than with college students $(d=0.23$, CI: 0.17 to 0.30$)$, suggesting that analyses of cultural differences between samples of college students may provide a conservative estimate of the nature of the difference between Japanese and Americans on IND. However, this shift was significant only at trend level; between-class effect $\left(Q_{\mathrm{B}}=2.80, p<.10\right)$ and effect size estimates for both nonstudent adults and college students were quite heterogeneous $\left(Q_{\mathrm{w}}=5.18, p<.03\right.$, and $Q_{\mathrm{w}}=514.03, p<.001$, respectively). Thus, even within samples (student and nonstudent) effect sizes were heterogeneous, and it is not possible to generalize findings either to student or to nonstudent populations.

\section{Results of International Meta-Analyses on COL}

\section{Regional Analyses}

As displayed in Figure 3, Americans were lower in COL than were others from all regions of the world, with the exception of English-speaking countries. We found it surprising that Americans were lower in COL than Europeans were, befitting the idea of a uniquely American way of being (high individualism and low collectivism) but challenging the notion of a single "Western" culture. In addition, effect sizes for Asian regions were similar to those for European regions, and we found large effects only for comparisons with Africa. Almost half of all studies focused on comparisons between East Asian regions and America. However, there were considerable within-region differences as revealed by the significant homogeneity statistic $Q$ for each regional comparison (all $p \mathrm{~s}<.00001$ ). We turned to more fine-grained crossnational analyses to unpack this regional heterogeneity.

\section{Country-Level Analyses}

As shown in Figure 4, findings across the 50 country comparisons confirmed the general conclusion that relative to others, Americans were lower in COL. There were notable exceptions, though, showing higher American COL in comparison to New Zealand, France, Singapore, Tanzania, Egypt, Costa Rica, and 


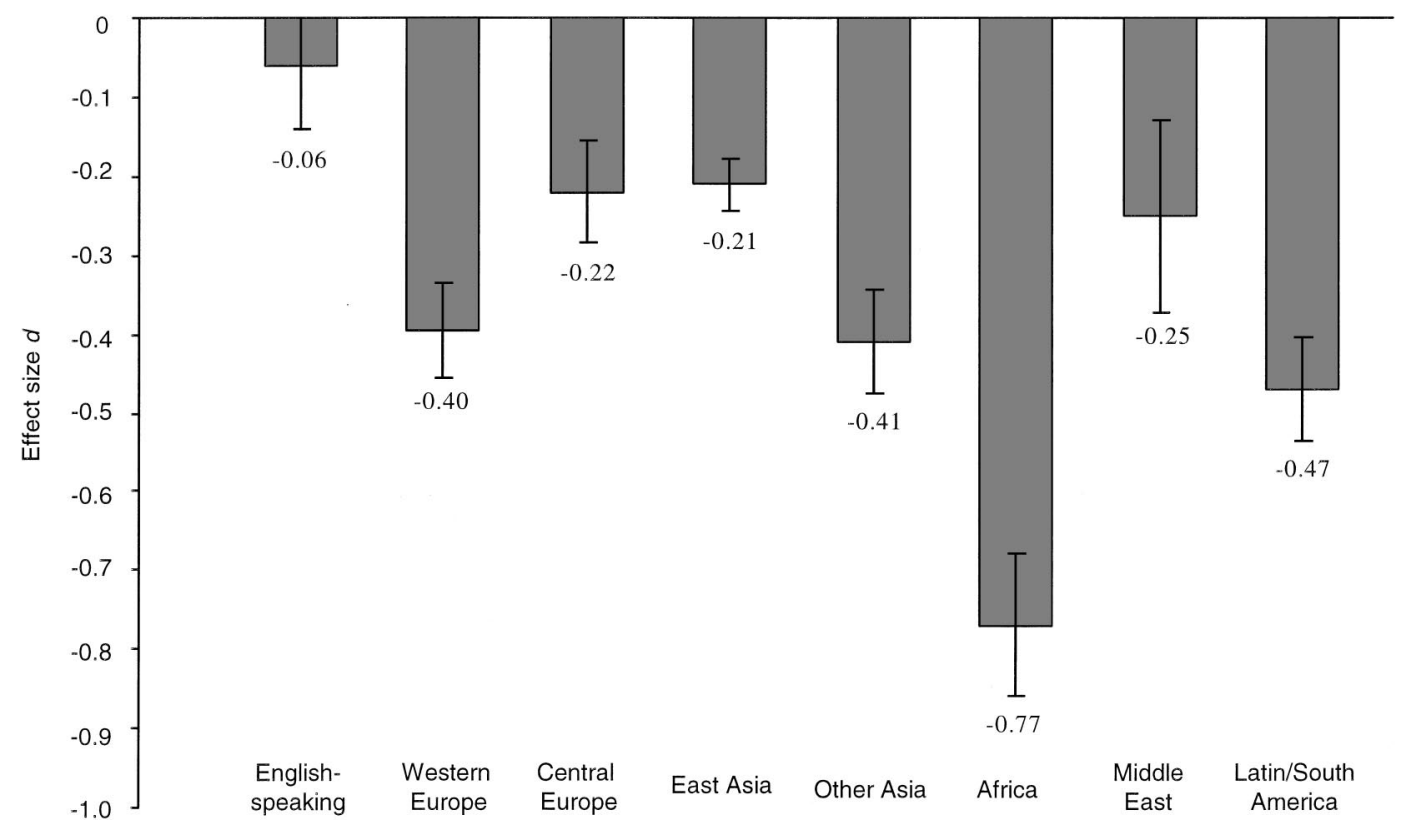

Figure 3. International meta-analysis on collectivism: Comparing samples from America and eight regions.

Venezuela. These exceptions were mostly due to effect sizes from single-study comparisons, and we were unable to assess the generality of these effects. The most remarkable findings were, first, the slightly lower COL in Japan compared with the United States and, second, the lack of difference between Korea and the United States in COL.

To examine overall effects and moderators of these effects in more detail, we meta-analytically synthesized research findings for the subset of country comparisons for which information from more than one study was available. As expected, Americans reported significantly lower COL than participants from most other countries. Table 5 summarizes these results. Moreover, unlike the predominantly small effects for IND, we found some large effect sizes for COL in comparisons with Israel, Nigeria, and Taiwan. We found moderate effects for India, Mexico, and PR China and small effects for Brazil, Hong Kong, and Indonesia. In addition, North Americans were not significantly different in COL from those European and English-speaking countries for which effect sizes could be estimated on the basis of more than a single study, namely Australia, Germany, and Poland.

Truly startling findings emerged for Korea and Japan: Americans were significantly higher in COL than Japanese were and were not significantly different in COL from Koreans. As we discuss in the section on psychological implications of IND-COL, the absence of a COL effect in Korea and its reversal in Japan is particularly noteworthy. Cross-cultural attribution research often focuses on Korean comparisons, and self-concept research often relies on Japanese comparisons, assuming marked differences in IND and COL without testing them. Finally, as was the case for IND comparisons, we found significant homogeneity coefficients, highlighting the necessity for moderator analyses.

Scale reliability moderator analyses. First, we examined the possible moderating role of scale reliability. The minimum required number of studies (two with low and two with high scale reliability) was available for Hong Kong, Japan, Korea, and PR China comparisons. For COL, reliable scales showed significantly larger effects - this contrasts to the lack of a systematic effect of reliability in the IND moderator analyses. As displayed in Table 6, higher scale reliability was associated with significantly increased effect sizes in Hong Kong-U.S. and PR China-U.S. comparisons. Americans were significantly lower in COL than Hong Kong and PR Chinese, and the size of this effect was significantly larger when reliable scales were used. With regard to Korea-U.S. comparisons, Americans were significantly lower in COL (small effect size) only in studies that used scales that are more reliable.

Figure 4 (opposite). International meta-analysis on collectivism: Comparing samples from America and 50 countries. Country comparisons are grouped by region, with countries appearing alphabetically within region. Light bars show effect sizes based on a single data point (a single-sample comparison), and dark bars show effect sizes based on multiple data points. These latter bars represent the countries for which a statistical integration of the available effect sizes is provided and, where possible, moderator analyses were performed. PR China $=$ People's Republic of China. 


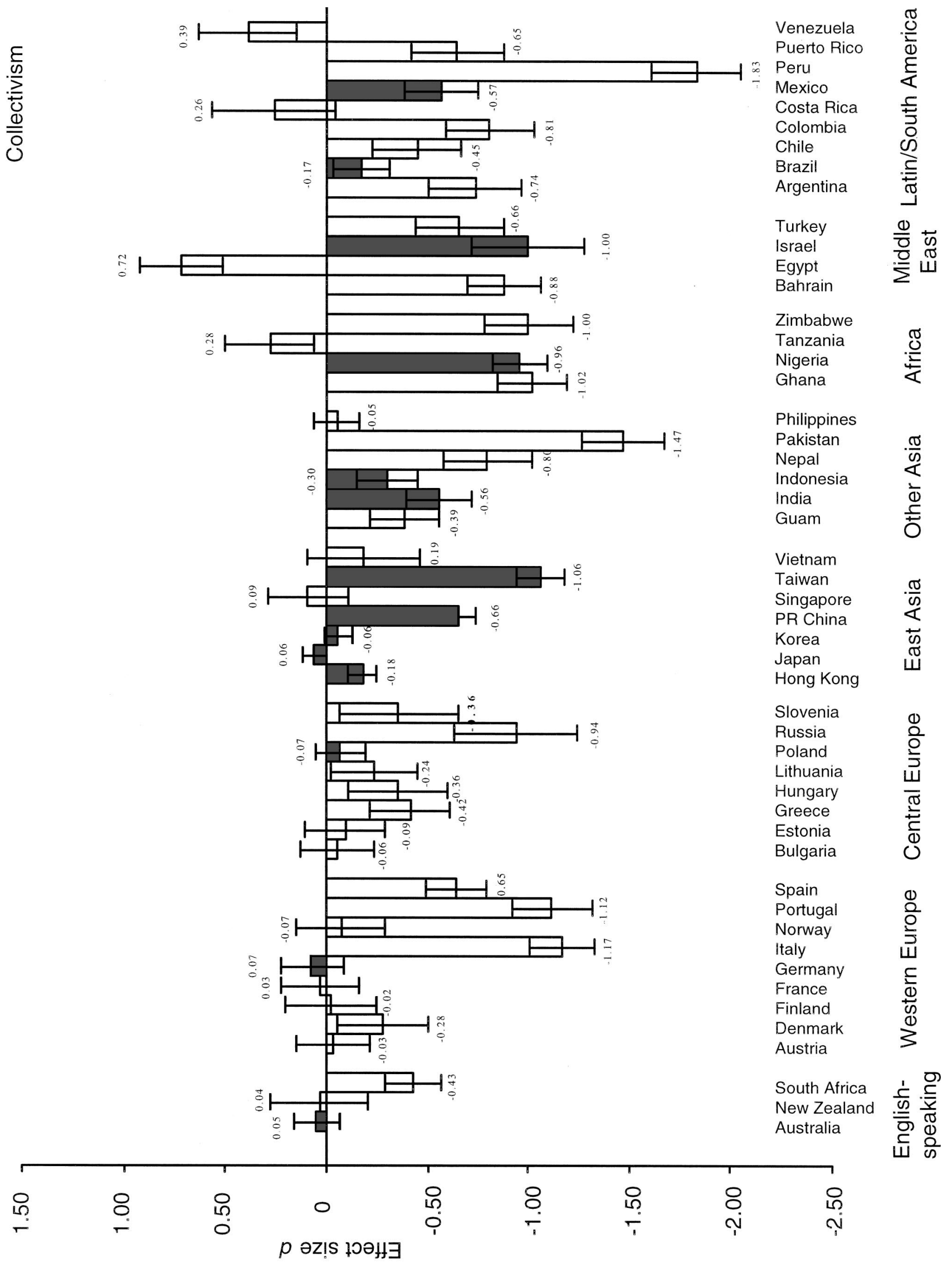


Table 5

International Meta-Analyses: Overall Collectivism Results

\begin{tabular}{|c|c|c|c|c|}
\hline Country & $n$ & $\begin{array}{l}\text { Mean weighted } \\
\text { effect size } \\
\left(d_{i+}\right)\end{array}$ & $95 \% \mathrm{CI}$ & $\begin{array}{l}\text { Homogeneity } \\
\text { within } \\
\text { country }\left(Q_{\mathrm{wi}}\right)\end{array}$ \\
\hline Australia & 3 & 0.05 & $-0.06 / 0.16$ & 1.38 \\
\hline Brazil & 2 & -0.17 & $-0.31 /-0.02$ & $28.22 * * *$ \\
\hline Germany & 2 & 0.07 & $-0.09 / 0.23$ & $95.14 * * *$ \\
\hline Hong Kong & 12 & -0.18 & $-0.25 /-0.11$ & $269.64 * * *$ \\
\hline India & 3 & -0.56 & $-0.72 /-0.40$ & 0.46 \\
\hline Indonesia & 2 & -0.30 & $-0.45 /-0.16$ & 0.04 \\
\hline Israel & 2 & -1.00 & $-1.28 /-0.72$ & 1.70 \\
\hline Japan & 17 & 0.06 & $0.002 / 0.11$ & $204.44 * * *$ \\
\hline Korea & 7 & -0.06 & $-0.13 / 0.02$ & $92.58 * * *$ \\
\hline Mexico & 3 & -0.55 & $-0.73 /-0.37$ & $215.41 * * *$ \\
\hline Nigeria & 3 & -0.96 & $-1.09 /-0.83$ & $83.64 * * *$ \\
\hline $\begin{array}{l}\text { People's Republic } \\
\text { of China }\end{array}$ & 9 & -0.66 & $-0.74 /-0.58$ & $164.90 * * *$ \\
\hline Poland & 4 & -0.07 & $-0.19 / 0.03$ & $48.35^{* * *}$ \\
\hline Taiwan & 3 & -1.06 & $-1.18 /-0.94$ & $136.25 * * *$ \\
\hline
\end{tabular}

Note. Positive values indicate higher American collectivism; negative values indicate lower American collectivism. Combined effect sizes that differ reliably from 0 are bold. $\mathrm{CI}=$ confidence interval. $* * * p<.001$

For Japan-U.S. comparisons, high reliability was associated with a reversal of the direction of the effect-Americans were higher in COL when comparisons used reliable scales but Americans and Japanese were not different in COL when comparisons used low-reliability scales. This finding is particularly important because even though Japan has received considerable attention by cultural psychologists, most available studies have involved lowreliability scales. Findings from these low-reliability studies have led researchers to assert higher American individualism (often termed independence) and lower American collectivism (often termed interdependence). The dramatic reversal that occurs when reliable scales are used is important because it highlights a validity problem in measurement of COL in studies with Japan. By definition, highly reliable scales have little random error; less reliable scales have more of this random error. Random error cannot itself produce reversal in direction of correlation, suggesting that for Japan-America comparisons, low- and high-reliability scales are not assessing the same underlying construct-a validity problem that further undermines assumptions of lower Japanese IND and higher Japanese COL.

Scale content moderator analyses. To explore whether observed effect sizes differed as a function of scale content within each country comparison, we also examined the moderating role of a content domain whenever the minimum number of studies (two with and two without a specific content category) was available. This allowed us to analyze scale content moderator effects for Hong Kong, Japan, Korea, PR China, and Poland, as shown in Table 7. In all of these comparisons, measurements of COL included "sense of duty to in-group"; thus, this component was constant across analyses. In addition, these scale content analyses were further limited because for Hong Kong and Poland, measurement of COL always included "relatedness to others" and the measurement of COL in Korea, Poland, and PR China always included "working in a group." In addition, "valuing group har- mony" was always present in Korea country comparisons, and "seeking others' advice" was always present in Poland country comparisons (refer to Table 1 for a description of each domain). Because COL components were constants in some country comparisons but not others, analyses are not necessarily comparable across countries. This heterogeneity of scale content hampers our ability to ascertain both whether countries that differ from the United States also differ from each other and also whether current effects would remain if researchers used different COL scales.

Despite these limitations, we draw a number of working conclusions. First, looking across all country comparisons, assessed content mattered. Second, countries differed in their responses to particular COL-related content. That is, whereas Americans are generally lower in COL, the size of the difference between Americans and others is reduced, and Americans may even report higher COL than others when COL is assessed with sense of "belonging to in-groups" and "seeking others' advice." Because duty to ingroup is the most commonly assessed component of COL, this finding suggests that belongingness and seeking others' advice do not fit with conceptions of COL as focused on duty to in-group. Perhaps for Americans, sense of belonging and seeking advice tap into feelings of choice about membership in the group and pleasure in relating to others-concepts more commensurate with conceptualizations of relationships developed within an individualistic value frame.

Turning to specific country comparisons, we first examined whether scale content moderated the extent to which Americans were significantly lower in COL than were Hong Kong Chinese. Clearly how COL was operationalized mattered; Americans were lower in COL when researchers assessed it with items pertaining to group harmony, defining the self in context, and valuing hierarchy and group goals. In fact, when COL scales did not contain group harmony, valuing hierarchy, and group goals, respondents from the United States and Hong Kong were no different in COL. Moreover, when COL did not contain defining oneself in context, Americans were significantly higher in COL than people from Hong Kong, though effect sizes were small. When researchers included belonging to one's in-groups in assessment of COL, the direction of the effect shifted and Americans were significantly higher in COL. Similarly, when researchers included seeking advice of others in assessment of COL, the difference in COL between Hong Kong and the United States was smaller (trend level). These findings suggest that factors other than the assumed latent COL construct may determine belongingness and seeking advice and that, at least for U.S.-Hong Kong comparisons, differences in accepting hierarchy and group goals, striving to maintain group harmony, and defining oneself contextually all do seem to relate to an underlying latent COL construct.

As seen in Table 7, the situation for Japan is complex. Americans were higher in COL than Japanese in most circumstances, including when scales included content that seemed to relate to a latent COL construct for U.S.-Hong Kong comparisons (accepting hierarchy, striving to maintain group harmony, defining oneself contextually) as well as sense of belonging to groups. Moreover, the expected finding of higher Japanese COL occurred only when scales contained preference for working in a group and did not contain striving to maintain group harmony (Americans were higher in COL than Japanese when COL scales did not contain working in groups). Even when scales showed higher Japanese 
Table 6

International Meta-Analyses: Collectivism Scale Reliability Moderator Analyses

\begin{tabular}{|c|c|c|c|c|c|}
\hline Country & $\begin{array}{l}\text { Between-class } \\
\text { effect }\left(Q_{\mathrm{B}}\right)\end{array}$ & $n$ & $\begin{array}{l}\text { Mean weighted } \\
\text { effect size }\left(d_{i+}\right)\end{array}$ & $95 \% \mathrm{CI}$ & $\begin{array}{l}\text { Homogeneity within } \\
\text { class }\left(Q_{\mathrm{wi}}\right)\end{array}$ \\
\hline Hong Kong & $21.69 * *$ & & & & \\
\hline Low reliability & & 5 & -0.13 & $-0.22 /-0.03$ & $163.02 * * *$ \\
\hline High reliability & & 4 & -0.49 & $-0.61 /-0.37$ & $34.66 * * *$ \\
\hline Japan & $3.56 \dagger$ & & & & \\
\hline Low reliability & & 10 & 0.01 & $-0.07 / 0.07$ & $146.09 * * *$ \\
\hline High reliability & & 5 & 0.12 & $0.02 / 0.23$ & $39.80 * *$ \\
\hline Korea & $3.67 *$ & & & & \\
\hline Low reliability & & 3 & 0.04 & $-0.07 / 0.15$ & $73.91 * * *$ \\
\hline High reliability & & 3 & -0.12 & $-0.23 / 0.00$ & $10.74 * *$ \\
\hline People's Republic of China & $3.89 *$ & & & & \\
\hline Low reliability & & 4 & -0.60 & $-0.70 /-0.51$ & $97.75 * * *$ \\
\hline High reliability & & 5 & -0.77 & $-0.91 /-0.64$ & $63.26^{* * *}$ \\
\hline
\end{tabular}

Note. Positive values indicate higher American collectivism; negative values indicate lower American collectivism. Studies with Cronbach's $\alpha<.70$ were classified as low reliability; studies with Cronbach's $\alpha \geq .70$ were classified as high reliability. Studies for which no reliability information was available were not included. Combined effect sizes that differ reliably from 0 are bold. $\mathrm{CI}=$ confidence interval.

$\dagger p<.10$ (marginally significant). $* p<.05$. *** $p<.01$. *** $p<.001$.

COL, effect sizes were small. Consequently, if Americans are in fact lower in COL than Japanese, the kind of COL this reflects may be focused particularly on a Japanese preference for working in groups. Of course, it is entirely possible that if researchers studied people other than college students, other differences would emerge.

Whereas Hong Kong and Japan afforded many component comparisons, fewer comparisons were available for Korea, PR China, and Poland because there were fewer studies comparing Americans and these countries and because scales used in these country comparisons were more homogeneous in content. For Korea-United States comparisons, content mattered; when researchers included "relatedness," Koreans had higher COL scores than Americans, but when it was not included, the direction of this effect reversed and Americans had higher COL scores than Koreans. Although PR Chinese were more collectivistic than Americans were regardless of how researchers assessed COL, content mattered to some extent in these country comparisons as well. As was the case for Hong Kong comparisons, "maintaining group harmony" seemed central to COL for PR Chinese. When researchers included maintaining group harmony in their assessment of COL, the PR Chinese-American effect size jumped from nonsignificant to significant and large. Seeking advice and relatedness (always assessed together) dampened rather than sharpened the PR China-U.S. difference-effect sizes were significantly higher when COL scales did not include these components. Finally, in the case of Poland, it was not possible to draw simple conclusions about the nature of scale content effects. All Polish studies included four of the eight components of COL, and two components were included in two studies but not included in two others. Thus, although we could perform an analysis, its interpretation is opaque.

Overall, the meta-analyses showed Americans to be generally lower in COL. Analyses of scale content suggested that the nature of the difference between Americans and others depends on scale content. Though impossible to state conclusively given the nature of the data, consistent between-country differences lead us to conclude that the essential cross-cultural difference underlying lower American COL lies in lower American valuation of group harmony and duty to the group. For Americans, relatedness, seeking others' advice, and feeling a sense of group belonging do not seem determined by this core collectivist worldview but rather are all forms of American-style relationality.

Sample composition moderator analyses. Given that research based on university students (who may not be representative of the country as a whole) dominated the empirical base, we were interested in examining whether effect sizes would differ in comparisons of student and nonstudent samples. For a number of reasons, it is possible that $\mathrm{COL}$ is lower among college students. For example, COL may be lower among college students because they are drawn from higher socioeconomic status subgroups, because university education provides familiarity with Western culture, or because university education reduces boundedness to in-groups. Our ability to test this hypothesis was limited, and sufficient data were available only for comparisons involving PR China. Four PR China comparisons involved students and five involved nonstudent adults. Effect sizes differed for these two populations $\left(Q_{\mathrm{b}}=7.22\right.$, $p<.01)$, with the difference for students $(d=-0.74$, CI: -0.84 to -0.64$)$ being larger than that for adults $(d=-0.52$, CI: -0.65 to -0.38$)$. Both effect sizes were heterogeneous $\left(Q_{\mathrm{w}}=29.29\right.$ and $Q_{\mathrm{w}}=128.39$, respectively; both $\left.p \mathrm{~s}<.001\right)$. Contrary to expectations, the U.S.-PR China difference in COL was larger when students rather than nonstudent adults were included. Note that the nonstudent adults themselves were not representative samples of PR Chinese adults but rather primarily business managers. Thus, the existing database cannot provide much insight into the question of whether students and nonstudents differ in COL.

\section{Graphing the Relationship Between IND and COL in Cross-Country Comparisons}

\section{Regional Analyses}

Another way to evaluate the extent that Americans are a gold standard of high individualism and low collectivism is to plot IND 
Table 7

International Meta-Analyses: Collectivism Scale Content Moderator Analyses

\begin{tabular}{|c|c|c|c|c|c|}
\hline Country & $\begin{array}{c}\text { Between-class } \\
\text { effect }\left(Q_{\mathrm{B}}\right)\end{array}$ & s & $\begin{array}{l}\text { Mean weighted } \\
\text { effect size } \\
\left(d_{i+}\right)\end{array}$ & $95 \% \mathrm{CI}$ & $\begin{array}{l}\text { Homogeneity within } \\
\text { class }\left(Q_{\mathrm{wi}}\right)\end{array}$ \\
\hline \multicolumn{6}{|l|}{ Hong Kong (duty, related) } \\
\hline Advice & $3.37 \dagger$ & & & & \\
\hline Included & & 10 & -0.16 & $-0.23 /-0.08$ & $222.01 * * *$ \\
\hline Not included & & 2 & -0.38 & $-0.62 /-0.15$ & $44.36 * * *$ \\
\hline Harmony & $9.89 * *$ & & & & \\
\hline Included & & 8 & -0.25 & $-0.34 /-0.17$ & $181.04 * * *$ \\
\hline Not included & & 4 & -0.01 & $-0.14 / 0.12$ & $78.71 * * *$ \\
\hline Context & $89.52 * * *$ & & & & \\
\hline Included & & 3 & -0.61 & $-0.72 /-0.49$ & $7.69 *$ \\
\hline Not included & & 9 & 0.09 & $0.002 / 0.18$ & $181.04 * * *$ \\
\hline Belong & $72.53 * * *$ & & & & \\
\hline Included & & 3 & 0.41 & $0.26 / 0.55$ & $13.22 * *$ \\
\hline Not included & & 9 & -0.34 & $-0.42 /-0.27$ & $178.89 * * *$ \\
\hline Hierarchy/group ${ }^{a}$ & $61.79 * * *$ & & & & \\
\hline Included & & 4 & -0.50 & $-0.61 /-0.39$ & $37.99 * * *$ \\
\hline Not included & & 8 & 0.07 & $-0.03 / 0.16$ & $169.86^{* * *}$ \\
\hline \multicolumn{6}{|l|}{ Japan (duty) } \\
\hline Advice & $5.89 *$ & & & & \\
\hline Included & & 12 & 0.01 & $-0.05 / 0.08$ & $183.03 * * *$ \\
\hline Not included & & 5 & 0.16 & $0.06 / 0.27$ & $16.52 * *$ \\
\hline Harmony & $33.37 * * *$ & & & & \\
\hline Included & & 13 & 0.13 & $0.07 / 0.19$ & $142.10 * * *$ \\
\hline Not included & & 4 & -0.30 & $-0.44 /-0.17$ & $28.97 * * *$ \\
\hline Related & $3.84 \dagger$ & & & & \\
\hline Included & & 12 & 0.09 & $0.03 / 0.16$ & $127.93 * * *$ \\
\hline Not included & & 5 & -0.03 & $-0.13 / 0.07$ & $72.67 * * *$ \\
\hline Context & $10.28 * *$ & & & & \\
\hline Included & & 4 & 0.24 & $0.12 / 0.37$ & $35.65 * * *$ \\
\hline Not included & & 13 & 0.01 & $-0.05 / 0.07$ & $158.50 * * *$ \\
\hline Belong & $9.05^{* * *}$ & & & & \\
\hline Included & & 4 & 0.19 & $0.09 / 0.30$ & $32.33^{* * *}$ \\
\hline Not included & & 13 & 0.00 & $-0.06 / 0.07$ & $163.06 * * *$ \\
\hline Hierarchy & $8.21 * * *$ & & & & \\
\hline Included & & 5 & 0.19 & $0.09 / 0.30$ & $37.88 * * *$ \\
\hline Not included & & 12 & 0.01 & $-0.06 / 0.07$ & $158.35 * * *$ \\
\hline Group & $43.83 * * *$ & & & & \\
\hline Included & & 11 & -0.07 & $-0.14 /-0.003$ & $136.97 * * *$ \\
\hline Not included & & 6 & 0.34 & $0.24 / 0.44$ & $23.64 * * * *$ \\
\hline \multicolumn{6}{|c|}{ Korea (duty, harmony, group) } \\
\hline Related & $14.58 * * *$ & & & & \\
\hline Included & & 4 & -0.18 & $-0.28 /-0.07$ & $77.76 * * *$ \\
\hline Not included & & 2 & 0.15 & $0.02 / 0.28$ & 0.21 \\
\hline Belong & 0.40 & & & & \\
\hline Included & & 2 & -0.03 & $-0.15 / 0.09$ & $17.52 * * *$ \\
\hline Not included & & 4 & -0.08 & $-0.19 / 0.04$ & $74.52 * * *$ \\
\hline \multicolumn{6}{|c|}{ People's Republic of China (duty, group) } \\
\hline Advice/related ${ }^{\mathrm{a}}$ & $45.21 * * *$ & & & & \\
\hline Included & & 5 & -0.54 & $-0.63 /-0.45$ & $103.63 * * *$ \\
\hline Not included & & 4 & -1.27 & $-1.46 /-1.07$ & $16.63 * * *$ \\
\hline Harmony & $83.31 * * *$ & & & & \\
\hline Included & & 5 & -0.98 & $-1.09 /-0.88$ & $12.44 *$ \\
\hline Not included & & 4 & -0.23 & $-0.35 / 0.11$ & $69.15 * * *$ \\
\hline \multicolumn{6}{|c|}{ Poland (duty, related, advice, group) } \\
\hline Harmony/belong ${ }^{\mathrm{a}}$ & $6.57 *$ & & & & \\
\hline Included & & 2 & 0.18 & $-0.04 / 0.41$ & 0.50 \\
\hline Not included & & 2 & -0.16 & $-0.29 /-0.03$ & $41.28 * * *$ \\
\hline
\end{tabular}

Note. Positive values indicate higher American collectivism; negative values indicate lower American collectivism. In parentheses after the country name is the content that was contained in all or all but one research instrument used in studies that contribute to the country comparison. Only six of the seven Korean studies were included because no information on scale content was available for one study. Combined effect sizes that differ reliably from 0 are bold. $\mathrm{CI}=$ confidence interval.

${ }^{a}$ These two components were always assessed together and could not be analyzed separately.

$\dagger p<.10$ (marginally significant). $* p<.05$. ** $p<.01$. *** $p<.001$. 


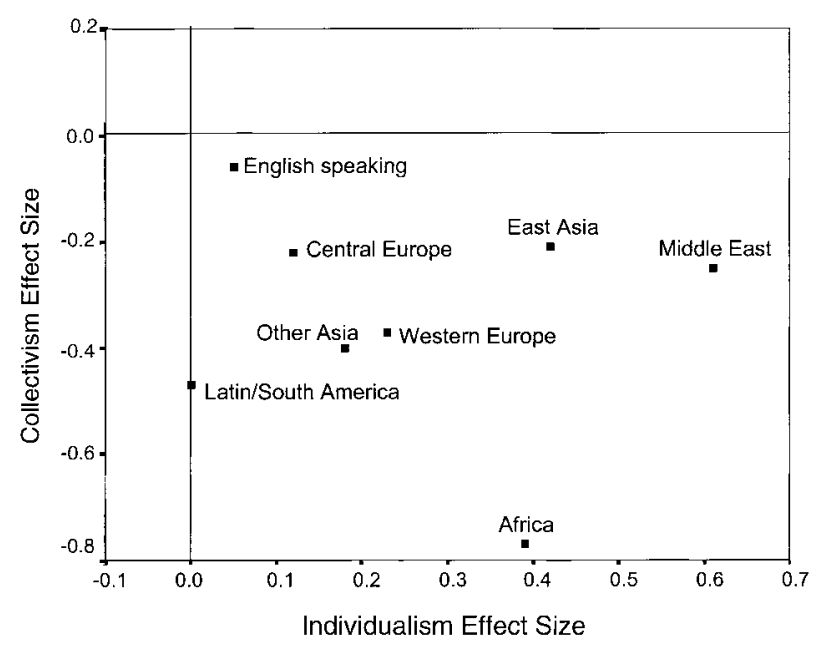

Figure 5. International meta-analyses: Plotting individualism and collectivism effect sizes from eight regions against each other.

and COL effect sizes against each other. Figure 5 presents just such a graphical regional analysis, plotting IND and COL effect size scores on a single set of axes. If Americans are simultaneously higher in IND and lower in COL than others are, then most regions of the world should be located in the lower right quadrant of Figure 5, with IND effect sizes being greater than zero and COL effect sizes being below zero. Scores do indeed cluster in the lower right quadrant.

Generally, Americans are both higher in IND and lower in COL than people from other regions of the world. Note, however, the following exceptions: Americans are indistinguishable on IND and COL from other English-speaking countries (Australia, New Zealand, and White South Africa) and though lower in COL, are no different in IND from South or Latin Americans. In addition, the hypothesized worldview difference-with Americans being higher in IND and lower in COL, was clearer in African and Middle Eastern comparisons than in East Asian comparisons. Although as a group East Asians were simultaneously lower in IND (moderate effect size) and higher in COL (small effect size) than were Americans, the size of this effect was larger in comparisons with African and Middle Eastern regions.

To determine the relation between IND and COL effect sizes, we performed a correlational analysis. We used Kendall's rankorder correlation as a measure of association because of the nonnormal distribution and the presence of outliers. IND and COL were unrelated $(\tau=.00)$, indicating that the size of difference between America and another region on IND was not predictive of the size of this difference on COL, and vice versa.

\section{Between-Country Analyses}

We repeated these graphical analyses at the country level, plotting IND and COL effect sizes against each other (see Figure 6). The assumption that Americans are uniquely high in IND and low in COL leads to the postulate that most countries will be in the lower right quadrant of the figure. We found this expected pattern with some notable exceptions. First, many but not all Latin and South American countries fell in the lower left quadrant, suggesting European Americans are lower in both IND and COL than people from most Latin American countries (exceptions are Venezuela and Costa Rica). Second, there is remarkable diversity within the East Asian countries that predominate cross-cultural research. Americans are higher in IND but no lower in COL than Japanese or Koreans. Americans are higher in IND and lower in COL than are Chinese people in PR China, Taiwan, and Hong Kong, and effects for these comparisons with Chinese are large. Third, only one country, New Zealand, fell into the upper left quadrant, being higher in IND and lower in COL than the United States.

As before, we assessed the relationship between IND and COL using Kendall's rank-order correlation. IND and COL effect sizes were not significantly correlated $(\tau=0.10, p=.34)$. Consistent with the independence of IND and COL in the regional analyses, knowing how different a country is in IND from the United States does not predict how much it differs in COL from the United States. These findings are inconsistent with assertions that IND and COL are negatively correlated when measured at the aggregate level and orthogonal only at the individual level (Leung, 1989; Triandis, 1989).

\section{Within-U.S. (Ethnic and Racial Group) Comparisons}

\section{Overview}

Next, we turned to the assumption that European Americans are higher in IND and lower in COL than are Americans of other

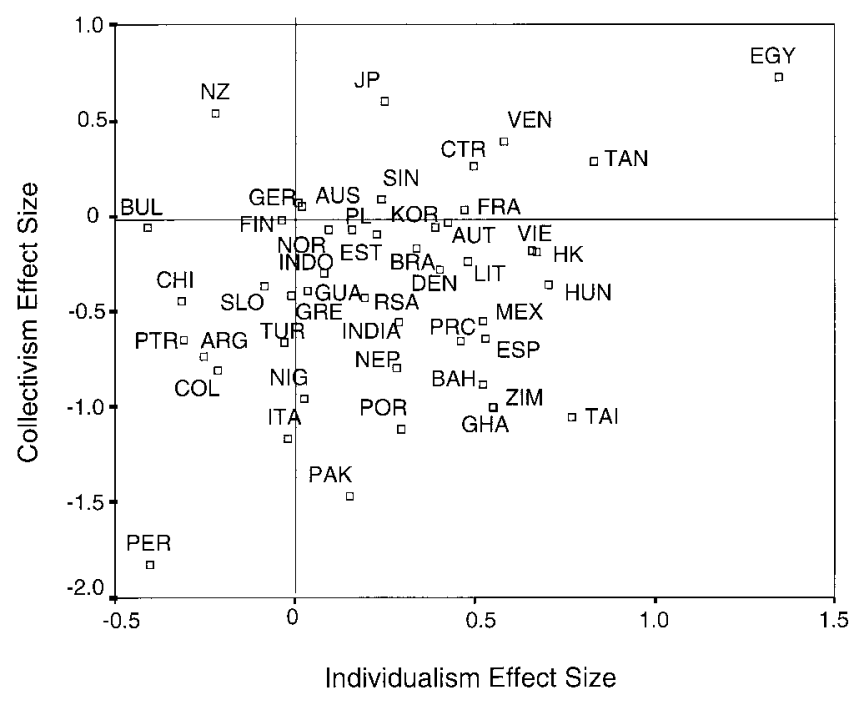

Figure 6. International meta-analyses: Plotting individualism and collectivism effect sizes from 46 countries against each other. ARG = Argentina; AUS $=$ Austria; AUT $=$ Australia; $\mathrm{BAH}=$ Bahrain; $\mathrm{BRA}=$ Brazil; $\mathrm{BUL}=$ Bulgaria $\mathrm{CHI}=$ Chile COL $=$ Colombia; $\mathrm{CTR}=$ Costa Rica; DEN = Denmark EGY = Egypt $;$ ESP $=$ Spain $;$ EST = Estonia; FIN = Finland; FRA = France; GER = Germany; GHA = Ghana; GRE = Greece; GUA = Guam; HK = Hong Kong; HUN = Hungary; INDO = Indonesia; ITA = Italy; JP = Japan; KOR = Korea; LIT = Lithuania; MEX $=$ Mexico; NEP = Nepal; NIG $=$ Nigeria; $\mathrm{NOR}=$ Norway; $\mathrm{NZ}=$ New Zealand; PAK $=$ Pakistan; PER $=$ Peru; PL $=$ Poland; $\mathrm{POR}=$ Portugal; PRC $=$ People's Republic of China; PTR = Puerto Rico; RSA = Republic of South Africa; TAI = Taiwan; TAN = Tanzania; TUR = Turkey; SIN = Singapore; SLO = Slovenia; VEN = Venezuela; VIE = Vietnam; ZIM = Zimbabwe. 
ethnic and racial groups. We found 21 articles and research reports that reported on 35 studies that assessed IND-COL and compared European Americans with African Americans, Asian Americans, and Latino Americans. Of the studies, 33 assessed both IND and COL, and 2 assessed COL only. Fourteen studies compared European Americans with one other ethnic and racial group, 3 compared European Americans with two ethnic and racial groups, and 18 compared European Americans with three ethnic and racial groups (for a total of 68 IND and 74 COL effect sizes). Appendix B summarizes each study used in the within-U.S. meta-analyses. Studies are organized alphabetically by author, and information is provided about year of publication, which groups were compared with European Americans, the number of participants in each sample, the percentage of women in the sample, sample composition (e.g., college students, managers), the IND or COL measurement used, effect size, and reliability codes for moderator analyses. This area of research is just emerging - studies are recent (published since 1990) and limited to the three largest nonEuropean American groups.

\section{Analyses Plan}

Generally, analyses were identical to the ones used for international comparisons. We present differences between European Americans and other Americans in IND in the following steps: summative analyses, between-group analyses, and moderator analyses. We then repeat these analyses for COL.

\section{Results of Within-U.S. Meta-Analyses on IND}

\section{Summative Analyses}

Because some researchers continue to contrast European Americans with heterogeneous groups of "Americans of color" (e.g., Gaines et al., 1997), we first contrasted European Americans to all other American groups, using all of the 68 available comparisons. Indeed, European Americans were higher in IND, but the effect size was small ( $d=0.08, \mathrm{CI}: 0.03$ to 0.13$)$ and heterogeneous $\left(Q_{w}\right.$ $=200.74, p<.001)$. The highly significant homogeneity coefficient underscores that generalizations about possible differences in IND between European Americans and individuals of other ethnicities are unwarranted. Therefore, we turned to comparisons between European Americans with African Americans, Latino Americans, and Asian Americans separately.

\section{Group Comparisons}

As summarized in Table 8, the assumption that European Americans are more individualistic than other Americans did not find general support. First, African Americans were significantly higher in IND than European Americans were. A nonsignificant homogeneity coefficient indicated that this effect, though small, consistently emerged across studies. Second, Latino Hispanic Americans did not differ significantly from European Americans in IND. This result is compatible with the international comparison findings in which Americans were generally no more individualistic than people from Latin America and were significantly lower in IND than Puerto Ricans. European Americans were only higher in IND when compared with Asian Americans, and the effect was small (congruent with the effect size in international comparisons).
Table 8

Within-U.S. Meta-Analyses: Overall Individualism Results

\begin{tabular}{|c|c|c|c|c|}
\hline Group & $n$ & $\begin{array}{l}\text { Mean weighted } \\
\text { effect size } \\
\left(\beta_{i+}\right)\end{array}$ & $95 \%$ CI & $\begin{array}{l}\text { Homogeneity } \\
\text { within group } \\
\left(Q_{\mathrm{wi}}\right)\end{array}$ \\
\hline African Americans & 19 & -0.31 & $-0.41 /-0.20$ & 25.54 \\
\hline Asian Americans & 28 & 0.24 & $0.18 / 0.30$ & $61.83 * * *$ \\
\hline Latino Americans & 21 & -0.01 & $-0.12 / 0.10$ & $31.64 *$ \\
\hline
\end{tabular}

Note. Positive values indicate higher European American individualism; negative values indicate lower European American individualism. Combined effect sizes that differ reliably from 0 are bold. $\mathrm{CI}=$ confidence interval.

$* p<.05 . \quad * * * p<.001$.

Furthermore, significant homogeneity coefficients for both Asian American and Latino American comparisons highlight the need to examine moderator variables. Although homogeneity coefficients were not significant for African Americans, we examined moderators for which sufficient data were available to shed further light on the processes behind these findings.

Scale reliability moderator analyses. As summarized in Table 9, we first examined the impact of scale reliability on the effect sizes of comparisons. Recall that for the international comparisons, this moderator analysis was inconclusive and scale reliability did not have consistent effects. For within-U.S. comparisons, we could only examine the influence of scale reliability on Asian American and Latino American comparisons. We could not test the effect of low scale reliability for comparisons with African Americans because all but one of the studies involving African Americans reported high scale reliability. For both Asian American and Latino American comparisons, increased scale reliability was associated with an increase in the effect size in the direction of greater European American IND. Even so, changes in effects were only at trend level for Asian American comparisons, whereas for Latino American comparisons, the direction of the effect shifted but neither high- nor low-reliability scale comparisons produced significant effect sizes. Thus, scale reliability did not provide a full explanation of variability, as can also be seen by the significant within-class coefficients.

Scale content moderator analyses. As with international comparisons, in assessing IND, researchers always included content about valuing personal independence; therefore we could not isolate the effect of valuing independence on our results. However, researchers varied in whether they included content about competition, personal uniqueness, privacy, self-knowledge, and direct communication (see Table 1 for a description of each domain) in assessing IND, so we assessed the effect of these in our moderator analyses. As summarized in Table 10, regardless of IND scale content, Latino Americans and European Americans did not differ in IND. However, for African American-European American and Asian American-European American comparisons, scale content mattered, influencing both effect size and direction.

In other words, the lack of difference between European and Latino Americans on IND was robust to scale content, but the significant difference between European Americans and African Americans and Asian Americans was not robust with regard to scale content. Content regarding personal uniqueness accentuated 
Table 9

Within-U.S. Meta-Analyses: Individualism Scale Reliability Moderator Analyses

\begin{tabular}{ccrccc}
\hline Group & $\begin{array}{c}\text { Between-class } \\
\text { effect }\left(Q_{\mathrm{B}}\right)\end{array}$ & $n$ & $\begin{array}{c}\text { Mean weighted } \\
\text { effect size }\left(d_{i+}\right)\end{array}$ & $95 \% \mathrm{CI}$ & $\begin{array}{c}\text { Homogeneity within } \\
\text { class }\left(Q_{\mathrm{wi}}\right)\end{array}$ \\
\hline Asian Americans & $3.19 \dagger$ & & & & \\
Low reliability & & 18 & $\mathbf{0 . 2 0}$ & $0.12 / 0.29$ & $29.22^{*}$ \\
High reliability & & 7 & $\mathbf{0 . 3 3}$ & $0.22 / 0.45$ & $19.64^{* *}$ \\
Latino Americans & $5.47^{*}$ & & & & \\
Low reliability & & 17 & -0.10 & $-0.23 / 0.03$ & $23.66 \dagger$ \\
High reliability & & 4 & 0.17 & $-0.01 / 0.36$ & 2.51 \\
\hline
\end{tabular}

Note. Positive values indicate higher European American individualism; negative values indicate lower European American individualism. Studies with Cronbach's $\alpha<.70$ were classified as low reliability; studies with Cronbach's $\alpha \geq .70$ were classified as high reliability. For the Asian American comparisons, three studies were omitted because they lacked reliability information. Combined effect sizes that differ reliably from 0 are bold. $\mathrm{CI}=$ confidence interval.

$\dagger p<.10$ (marginally significant). $* x<.05$. ** $p<.01$.

differences between these groups of Americans; content regarding privacy had different effects depending on the group studied; and content regarding competition and self-knowledge diminished differences between these groups of Americans.

When researchers included "valuing personal uniqueness" in their assessment of IND, effect size significantly increased. Compared with European Americans, Asian Americans rated themselves lower in IND and African Americans rated themselves higher in IND when personal uniqueness was included as part of the IND assessment. When researchers included "valuing privacy" in their assessment of IND, they saw a significantly increased effect size for comparisons between African Americans and European Americans but a reduced difference between Asian Americans and European Americans to the point of nonsignificance. When researchers included "personal competition" in their assessment of IND, they found no reliable difference between European Americans and Asian Americans and found smaller, though still significant, differences between European Americans and African Americans. Similarly, including "self-knowledge" in assessment of IND significantly decreased the difference between African Americans and European Americans. Although current data do not provide the basis for conclusive analyses, we interpret these findings to suggest that in America, individualism involves valuing personal independence and uniqueness. Although plausibly connected to a general construct such as IND, valuing competition, privacy, and self-knowledge do not necessarily load with INDand appear not to do so in the American context.

Taken as a whole, international and within-U.S. comparisons on IND show similarly small effect sizes that are dependent on scale content. Assessing IND in terms of valuing independence and personal uniqueness enhances effect sizes. We take this to mean that valuing independence and personal uniqueness are essential to differences in IND between European Americans and othersAfrican Americans, Asian Americans, and Japanese. Personal privacy, which enhanced the difference in IND in Japan-U.S. comparisons, did not have this effect when we compared European Americans and Asian Americans (though it did enhance the difference in IND between African Americans and European Americans).

Sample composition moderator analyses. All studies used college students except for two studies reported by Gaines et al.
(1997). We contrasted these two studies with the remaining studies in sample composition moderator analyses. We did not find significant effects, but trend-level effects for African Americans and the pattern of effects for Asian Americans suggest that the overall difference found between groups is due to differences between students rather than nonstudents (see Table 11). Clearly there is insufficient information to draw strong conclusions because the nonstudents in the Gaines et al. research cannot be assumed to represent the population at large.

\section{Results of Within-U.S. Meta-Analyses on COL}

\section{Summative Analyses}

Because researchers continue to contrast European Americans with heterogeneous groups of "Americans of color" (e.g., Gaines et al., 1997), we first contrasted European Americans to all other American groups, using all of the 74 available comparisons. European Americans were lower in COL than others, but the combined effect size was small $(d=-0.28$, CI: -0.32 to -0.23$)$ and heterogeneous $\left(Q_{\mathrm{w}}=192.14, p<.001\right)$. The highly significant homogeneity coefficient underscores that generalizations about differences between European Americans and individuals of other ethnicities are unwarranted. Therefore, we turned to comparisons of European Americans with African Americans, Latino Americans, and Asian Americans separately.

\section{Group Comparisons}

As presented in Table 12, European Americans are significantly lower in COL than Asian Americans and Latino Americans, though effects are small. However, European Americans and African Americans do not significantly differ in COL. Though not directly comparable, effects appeared generally more modest for within-U.S. than for cross-national comparisons. Significant homogeneity coefficients prompted us to proceed with moderator analyses, first focusing on the potential effects of scale reliability.

Scale reliability moderator analyses. To our surprise, as shown in Table 13, scale reliability had no significant effect on any comparison. This is unlike the international COL comparisons, in 
Table 10

Within-U.S. Meta-Analyses: Individualism Scale Content Moderator Analyses

\begin{tabular}{|c|c|c|c|c|c|}
\hline Group & $\begin{array}{l}\text { Between-class } \\
\text { effect }\left(Q_{\mathrm{B}}\right)\end{array}$ & $n$ & $\begin{array}{l}\text { Mean weighted } \\
\text { effect size } \\
\left(d_{i+}\right)\end{array}$ & $95 \% \mathrm{CI}$ & $\begin{array}{c}\text { Homogeneity } \\
\text { within class } \\
\left(Q_{\mathrm{wi}}\right)\end{array}$ \\
\hline \multicolumn{6}{|c|}{ African Americans (independent) } \\
\hline Self-know & $5.05 *$ & & & & \\
\hline Included & & 9 & -0.11 & $-0.31 / 0.08$ & 9.96 \\
\hline Not included & & 10 & -0.38 & $-0.51 /-0.26$ & $10.52 *$ \\
\hline Direct communicate & 0.98 & & & & \\
\hline Included & & 17 & -0.35 & $-0.47 /-0.22$ & $23.67 \dagger$ \\
\hline Not included & & 2 & -0.24 & $-0.41 /-0.06$ & 0.90 \\
\hline Unique & $3.03 \dagger$ & & & & \\
\hline Included & & 11 & -0.36 & $-0.48 /-0.23$ & 12.68 \\
\hline Not included & & 8 & -0.13 & $-0.36 / 0.09$ & 9.83 \\
\hline Private & $6.71 * *$ & & & & \\
\hline Included & & 3 & -0.57 & $-0.79 /-0.34$ & 0.08 \\
\hline Not included & & 16 & -0.35 & $-0.23 /-0.11$ & $18.74 * *$ \\
\hline Compete & $5.80^{*}$ & & & & \\
\hline Included & & 10 & -0.20 & $-0.33 /-0.06$ & 11.21 \\
\hline Not included & & 9 & -0.34 & $-0.54 /-0.14$ & 8.53 \\
\hline \multicolumn{6}{|c|}{ Asian Americans (independent) } \\
\hline Self-know & 0.71 & & & & \\
\hline Included & & 11 & 0.23 & $0.11 / 0.35$ & 12.34 \\
\hline Not included & & 16 & 0.29 & $0.21 / 0.36$ & $41.70 * * *$ \\
\hline Direct communicate & 0.09 & & & & \\
\hline Included & & 24 & 0.27 & $0.20 / 0.34$ & $52.87 * * *$ \\
\hline Not included & & 3 & 0.24 & $0.03 / 0.45$ & 1.79 \\
\hline Unique & $7.04 * *$ & & & & \\
\hline Included & & 19 & 0.30 & $0.23 / 0.37$ & $43.11 * * *$ \\
\hline Not included & & 8 & 0.05 & $-0.12 / 0.23$ & 4.60 \\
\hline Private & $9.17 * *$ & & & & \\
\hline Included & & 4 & 0.06 & $-0.10 / 0.21$ & 0.05 \\
\hline Not included & & 23 & 0.32 & $0.24 / 0.39$ & $45.06^{* *}$ \\
\hline Compete & $8.57 * *$ & & & & \\
\hline Included & & 9 & 0.07 & $-0.07 / 0.21$ & 4.79 \\
\hline Not included & & 18 & 0.32 & $0.24 / 0.39$ & $41.39 * * *$ \\
\hline \multicolumn{6}{|c|}{ Latino Americans (independent) } \\
\hline Direct & 0.03 & & & & \\
\hline Included & & 19 & -0.01 & $-0.12 / 0.11$ & $31.57 *$ \\
\hline Not included & & 2 & -0.04 & $-0.40 / 0.32$ & 0.03 \\
\hline Unique/self-know ${ }^{\mathrm{a}}$ & 0.03 & & & & \\
\hline Included & & 13 & 0.00 & $-0.13 / 0.12$ & 14.36 \\
\hline Not included & & 8 & -0.03 & $-0.25 / 0.19$ & $17.24 *$ \\
\hline Private & 0.06 & & & & \\
\hline Included & & 6 & 0.00 & $-0.15 / 0.16$ & $11.69^{*}$ \\
\hline Not included & & 15 & -0.02 & $-0.18 / 0.13$ & 19.89 \\
\hline Compete & 0.05 & & & & \\
\hline Included & & 9 & -0.03 & $-0.25 / 0.18$ & 17.28 \\
\hline Not included & & 12 & 0.00 & $-0.13 / 0.13$ & 14.30 \\
\hline
\end{tabular}

Note. Positive values indicate higher European American individualism; negative values indicate lower European American individualism. In parentheses after the group labels is the content that was contained in all or all but one of the research instruments used in studies that contribute to the group comparison. For one study involving Asian Americans, no scale content information was available. Combined effect sizes that differ reliably from 0 are bold. $\mathrm{CI}=$ confidence interval.

${ }^{a}$ In the case of comparisons with Latino Americans, all scales that assessed uniqueness also assessed selfknowledge; therefore, the two could not be analyzed separately.

$\dagger p<.10$ (marginally significant). $* p<.05$. ** $p<.01$. *** $p<.001$.

which higher reliability scales resulted in greater effects. Further examination of Table 13 shows significant intraclass heterogeneity only among more reliable scales, suggesting diversity within these studies due to other factors.

Scale content moderator analyses. With regard to scale content, researchers in all studies included both sense of duty to the group and relatedness in their assessment of COL. This means that all of our analyses of other COL content includes this content as constants and that within-U.S. and cross-cultural analyses of content are not comparable because different content was constant in these analyses.

Despite these limitations, we draw a number of conclusions. First, as seen in Table 14, content mattered more for comparisons between European Americans and both African Americans and 
Table 11

Within-U.S. Meta-Analyses: Individualism Sample Composition Moderator Analyses

\begin{tabular}{|c|c|c|c|c|c|}
\hline Group & $\begin{array}{c}\text { Between-class } \\
\text { effect }\left(Q_{\mathrm{B}}\right) \\
\end{array}$ & $n$ & $\begin{array}{c}\text { Mean weighted } \\
\text { effect size } \\
\left(d_{i+}\right)\end{array}$ & $95 \%$ CI & $\begin{array}{l}\text { Homogeneity } \\
\text { within class } \\
\left(Q_{\mathrm{wi}}\right) \\
\end{array}$ \\
\hline African Americans & $3.08 \dagger$ & & & & \\
\hline Students & & 17 & -0.34 & $-0.45 /-0.23$ & 22.44 \\
\hline Nonstudent adults & & 2 & -0.02 & $-0.36 / 0.33$ & 0.01 \\
\hline Asian Americans & 1.32 & & & & \\
\hline Students & & 26 & 0.25 & $0.19 / 0.31$ & $59.30 * * *$ \\
\hline Nonstudent adults & & 2 & 0.02 & $-0.36 / 0.40$ & 1.21 \\
\hline Latino Americans & $3.10 \dagger$ & & & & \\
\hline Students & & 19 & -0.02 & $-0.14 / 0.09$ & $29.11 *$ \\
\hline Nonstudent adults & & 2 & 0.16 & $-0.22 / 0.54$ & 1.70 \\
\hline
\end{tabular}

Note. Positive values indicate higher European American individualism; negative values indicate lower European American individualism. Studies with Cronbach's $\alpha<.70$ were classified as low reliability; studies with Cronbach's $\alpha \geq .70$ were classified as high reliability. For the Asian American comparisons, the three studies were omitted that lacked reliability information. Combined effect sizes that differ reliably from 0 are bold. $\mathrm{CI}=$ confidence interval.

$\dagger p<.10$ (marginally significant). $* p<.05$. $* * * p<.001$.

Latino Americans than for comparisons between European Americans and Asian Americans. Second, with one exception, European Americans were lower in COL only when researchers did not include seeking advice from others, defining the self contextually, and concern for status and hierarchy in their assessment of COL. Third, inclusion or exclusion of focus on group harmony, belonging to groups, and preference for group work in assessment of COL did not influence effect size.

In studies in which researchers did not assess seeking advice as part of COL, all minority groups were higher in COL than European Americans. In studies in which researchers did assess seeking advice as part of COL, both African Americans and Latino Americans were no different from European Americans, suggesting that these groups of Americans do not differ in the extent that they seek advice from others. This finding parallels the finding in international comparisons-Americans scored higher in COL when COL included seeking advice. Seeking advice seems to be an American way to connect and relate. With regard to defining the self contextually, Markus and Kitayama (1991) proposed that defining the self contextually is central to COL. However, including items tapping this issue in assessment of COL did not enhance betweengroup COL differences. In fact, the difference between African Americans and European Americans and the difference between Latino Americans and European Americans in COL disappeared

Table 12

Within-U.S. Meta-Analysis: Overall Collectivism Results

\begin{tabular}{|c|c|c|c|c|}
\hline Group & $n$ & $\begin{array}{c}\text { Mean weighted } \\
\text { effect size } \\
\left(\beta_{i+}\right)\end{array}$ & $95 \% \mathrm{CI}$ & $\begin{array}{l}\text { Homogeneity } \\
\text { within group } \\
\left(Q_{\mathrm{wi}}\right)\end{array}$ \\
\hline African Americans & 20 & -0.04 & $-0.14 / 0.06$ & $38.28 * *$ \\
\hline Asian Americans & 31 & -0.39 & $-0.45 /-0.33$ & $75.31 * * *$ \\
\hline Latino Americans & 23 & -0.21 & $-0.31 /-0.11$ & $39.94 *$ \\
\hline
\end{tabular}

Note. Positive values indicate higher European American collectivism; negative values indicate lower European American collectivism. Combined effect sizes that differ reliably from 0 are bold. $\mathrm{CI}=$ confidence interval. $* p<.05$. ** $p<.01$. *** $p<.001$. when researchers included defining the self contextually as part of the assessment of COL (and had no influence on the Asian American-European Americans comparison). This result suggests that defining the self contextually is not central to distinguishing among Americans on COL; though plausibly related to COL, defining the self contextually is likely to be a separate construct that is sometimes related to COL but also determined by other factors, such as for example, gender (cf. Cross \& Madson, 1997; Markus \& Oyserman, 1989). With regard to concern for status and hierarchy, again, the only time European Americans were lower in COL than African Americans and Latino Americans was when this component was not included. For Asian Americans, inclusion did not affect the size of the effect.

Finally, inclusion or exclusion of focus on group harmony, belonging to groups, and preference for group work did not influence effect size. This suggests that though plausibly related to collectivism, valuing in-group harmony, belonging to groups, and working in groups are multiply determined and not consistently associated with collectivism in the United States. We therefore interpret our meta-analytic results to suggest that duty to in-group is the core feature of the between-group differences associated with empirically assessed COL. Group harmony, associated with this core feature in international comparisons, does not appear to have this consistent association in within-U.S. comparisons.

Sample composition moderator analyses. We were able to compare two studies that relied on a nonstudent population with other studies that sampled students. As presented in Table 15, we did not find statistically reliable differences in the effect sizes. No strong conclusion about levels of COL among nonstudent Americans is warranted.

\section{Graphing the Relationship Between IND and COL in Within-U.S. Comparisons}

As for the international comparisons, we plotted IND and COL effect sizes against each other. If European Americans are higher in IND and lower in COL than Americans belonging to other ethnic or racial groups, data points would cluster in the right of 
Table 13

Within-U.S. Meta-Analyses: Collectivism Scale Reliability Moderator Analyses

\begin{tabular}{cccccc}
\hline Group & $\begin{array}{c}\text { Between-class } \\
\text { effect }\left(Q_{\mathrm{B}}\right)\end{array}$ & $n$ & $\begin{array}{c}\text { Mean weighted } \\
\text { effect size }\left(d_{i+}\right)\end{array}$ & $95 \%$ CI & $\begin{array}{c}\text { Homogeneity within } \\
\text { class }\left(Q_{\text {wi }}\right)\end{array}$ \\
\hline African Americans & 0.37 & & & & \\
$\quad$ Low reliability & & 8 & 0.00 & $-0.17 / 0.17$ & 11.48 \\
$\quad$ High reliability & & 12 & -0.06 & $-0.17 / 0.05$ & $26.43^{* *}$ \\
Asian Americans & 0.00 & & & & \\
$\quad$ Low reliability & & 9 & $\mathbf{- 0 . 4 2}$ & $-0.52 /-0.32$ & 5.11 \\
$\quad$ High reliability & 0.20 & 19 & $\mathbf{- 0 . 4 2}$ & $-0.51 /-0.33$ & $40.27^{* *}$ \\
Latino Americans & & 17 & $\mathbf{- 0 . 2 5}$ & $-0.45 /-0.05$ & 4.52 \\
Low reliability & & 6 & $\mathbf{- 0 . 2 0}$ & $-0.32 /-0.07$ & $35.23^{* *}$ \\
\hline High reliability & & & &
\end{tabular}

Note. Positive values indicate higher European American collectivism; negative values indicate lower European American collectivism. Studies with Cronbach's $\alpha<.70$ were classified as low reliability; studies with Cronbach's $\alpha \geq .70$ were classified as high reliability. A few studies had to be omitted in this analysis because of missing reliability information. Combined effect sizes that differ reliably from 0 are bold. $\mathrm{CI}=$ confidence interval.

$* * p<.01$.

Figure 7. An examination of Figure 7 clearly shows that this is not the case. Of the three groups we compared with European Americans, only Asian Americans were lower in IND and higher in COL than were European Americans. In these comparisons, we found a perfect relation between IND and COL $(\tau=-1.00)$. This means that American ethnic or racial groups higher in COL than European Americans are also lower in IND than European Americans. This finding is of note because we did not find a relationship between IND and COL in our international analyses; however, given that the within-U.S. analyses rest on three data points, significance testing is noninformative.

\section{Summary of International and Within-U.S. Meta-Analytic Findings}

A basic aim of the present review is to address the question, Are European Americans higher in IND (and lower in COL) than others? Given the present database, the answer is a qualified yes. Americans tend to be higher than others in IND and lower in COL, but there are several caveats. First, effect sizes tend to be small. Second, effects do not hold for Latin or South Americans or for African Americans. Third, effects for Asians and East Asians are diverse, with the largest effects occurring for people of Chinese origin and the smallest effects for Japanese and Koreans. Fourth, it is not entirely clear whether high IND and low COL is an American or a Western way of being because research to date has not sufficiently documented the nature of differences between European and English-speaking countries. Fifth, little research is available for many countries, and more is required if we are to understand the multifaceted nature of cultural ways of being connected and related to others, as opposed to seeking harmony or feeling obligated to them.

When researchers use sense of belonging to in-groups and seeking others' advice to assess COL, Americans rate themselves as relatively collective. When researchers use duty to in-group instead of these other ways of being related to assess COL, Americans rate themselves as quite low in COL. We interpret these findings to mean that belonging and seeking advice are American ways of being related and that this way of being interdependent is not incongruent with American individualism. Therefore, we propose separating assessment of feelings of belonging and connecting from feeling duty to in-group. By assessing relationality separately from COL, a better understanding of differences between Americans and Japanese may arise. As summarized in the international scale content moderator analyses section, AmericanJapanese COL comparisons suggest that Americans are higher or no different from Japanese in COL except under a few, and as yet unclear, circumstances. In contrast, Americans are robustly lower in COL than people of Chinese origin. In addition, both regional and country analyses show small differences between Americans and Western or Central Europeans, bolstering the assumption that there is a "Western" cultural frame. The meta-analyses also suggest the need to better understand the "Latino" cultural perspective because some of these countries (Argentina, Chile, Colombia, and Puerto Rico) are higher in IND and lower in COL than are European Americans.

The combination of small differences in IND and no COL difference (for Korea-U.S.) or small COL difference in the direction of higher U.S. COL (Japan-U.S.) is problematic for the large research base in areas such as self-concept that assume Japanese and Koreans are simultaneously higher in COL and lower in IND than are Americans. Although these results for Japan and Korea are surprising, we found additional corroboration of this noneffect in two reviews of cross-cultural research on Japanese and Americans (Matsumoto, 1999; Takano \& Osaka, 1999). Working with fewer studies, these authors also provided evidence that Americans and Japanese are not different in IND. Although researchers should not assume that all East Asians are lower in IND and higher in COL than Americans, they can make this assumption about East Asians of Chinese origin-from PR China, Taiwan, and Hong Kong - for whom we found robust effects. Indeed, according to the 2000 U.S. Census (U.S. Department of Commerce, 2001), people of Chinese origin form the largest group among Asian Americans, perhaps explaining this effect as well. Our results also make clear that there is no justification for lumping together minority Americans for the purpose of studying differences in individual or collective orientation. 
Table 14

Within-U.S. Meta-Analyses: Collectivism Scale Content Moderator Analyses

\begin{tabular}{|c|c|c|c|c|c|}
\hline Group & $\begin{array}{c}\text { Between-class } \\
\text { effect }\left(Q_{\mathrm{B}}\right)\end{array}$ & $n$ & $\begin{array}{l}\text { Mean weighted } \\
\text { effect size } \\
\left(d_{i+}\right)\end{array}$ & $95 \% \mathrm{CI}$ & $\begin{array}{c}\text { Homogeneity within } \\
\text { class }\left(Q_{\mathrm{wi}}\right)\end{array}$ \\
\hline \multicolumn{6}{|c|}{ African Americans (duty, related) } \\
\hline Advice & $11.19 * * *$ & & & & \\
\hline Included & & 11 & 0.05 & $-0.06 / 0.16$ & 12.31 \\
\hline Not included & & 9 & -0.34 & $-0.54 /-0.14$ & $14.77 \dagger$ \\
\hline Context & $3.83 \dagger$ & & & & \\
\hline Included & & 7 & -0.07 & $-0.08 / 0.21$ & $12.13 \dagger$ \\
\hline Not included & & 13 & -0.13 & $-0.26 / 0.003$ & $22.31 *$ \\
\hline Hierarchy & $6.57 *$ & & & & \\
\hline Included & & 7 & 0.10 & $-0.04 / 0.25$ & 7.73 \\
\hline Not included & & 13 & -0.15 & $-0.28 /-0.02$ & $23.98 *$ \\
\hline Harmony & 2.45 & & & & \\
\hline Included & & 9 & 0.06 & $-0.09 / 0.21$ & 7.78 \\
\hline Not included & & 11 & -0.10 & $-0.23 / 0.02$ & $28.04 * *$ \\
\hline Belong & 0.53 & & & & \\
\hline Included & & 3 & -0.03 & $-0.19 / 0.26$ & 0.08 \\
\hline Not included & & 17 & -0.06 & $-0.17 / 0.05$ & $37.67 * *$ \\
\hline Group & 0.19 & & & & \\
\hline Included & & 14 & -0.05 & $-0.16 / 0.06$ & $26.61 *$ \\
\hline Not included & & 6 & 0.00 & $-0.21 / 0.21$ & $11.47 *$ \\
\hline \multicolumn{6}{|c|}{ Asian Americans (duty, related) } \\
\hline Advice & $3.63 \dagger$ & & & & \\
\hline Included & & 20 & -0.38 & $-0.45 /-0.31$ & $29.75 \dagger$ \\
\hline Not included & & 10 & -0.52 & $-0.64 /-0.40$ & $35.43 * * *$ \\
\hline Context & 0.01 & & & & \\
\hline Included & & 15 & -0.42 & $-0.50 /-0.34$ & $30.24 * *$ \\
\hline Not included & & 15 & -0.41 & $-0.51 /-0.31$ & $38.57 * * *$ \\
\hline Hierarchy & 0.32 & & & & \\
\hline Included & & 16 & -0.43 & $-0.51 /-0.35$ & $31.74 * *$ \\
\hline Not included & & 14 & -0.39 & $-0.49 /-0.28$ & $36.75 * * *$ \\
\hline Harmony & 0.40 & & & & \\
\hline Included & & 20 & -0.43 & $-0.50 /-0.36$ & $33.39 *$ \\
\hline Not included & & 10 & -0.38 & $-0.51 /-0.24$ & $35.02 * * *$ \\
\hline Belong & 0.19 & & & & \\
\hline Included & & 5 & -0.39 & $-0.52 /-0.26$ & 1.97 \\
\hline Not included & & 25 & -0.42 & $-0.49 /-0.35$ & $66.65 * * *$ \\
\hline Group & 0.66 & & & & \\
\hline Included & & 19 & -0.44 & $-0.52 /-0.36$ & $41.65^{* *}$ \\
\hline Not included & & 11 & -0.38 & $-0.48 /-0.28$ & $26.50 * *$ \\
\hline \multicolumn{6}{|c|}{ Latino Americans (duty, related) } \\
\hline Advice & $15.52 * * *$ & & & & \\
\hline Included & & 13 & -0.07 & $-0.19 / 0.06$ & 11.83 \\
\hline Not included & & 10 & -0.51 & $-0.69 /-0.33$ & 12.59 \\
\hline Context & $11.93 * * *$ & & & & \\
\hline Included & & 6 & 0.24 & $-0.03 / 0.52$ & 12.13 \\
\hline Not included & & 17 & -0.28 & $-0.39 /-0.17$ & $25.72 \dagger$ \\
\hline Hierarchy & $3.01 \dagger$ & & & & \\
\hline Included & & 7 & -0.02 & $-0.26 / 0.21$ & $14.81 *$ \\
\hline Not included & & 16 & -0.25 & $-0.37 /-0.14$ & 22.13 \\
\hline Harmony & $2.93 \dagger$ & & & & \\
\hline Included & & 13 & -0.15 & $-0.27 /-0.03$ & $19.40 \dagger$ \\
\hline Not included & & 10 & -0.35 & $-0.53 /-0.16$ & $17.61^{*}$ \\
\hline Belong & 1.42 & & & & \\
\hline Included & & 6 & -0.14 & $-0.29 / 0.01$ & 0.08 \\
\hline Not included & & 17 & -0.27 & $-0.40 /-0.13$ & $35.06 * *$ \\
\hline Group & $3.14 \dagger$ & & & & \\
\hline Included & & 17 & -0.26 & $-0.37 /-0.14$ & $27.95^{*}$ \\
\hline Not included & & 6 & -0.02 & $-0.25 / 0.21$ & $8.85^{*}$ \\
\hline
\end{tabular}

Note. Positive values indicate higher European American collectivism; negative values indicate lower European American collectivism. In parentheses after the group labels is the content that was contained in all or all but one of the research instruments used in studies that contribute to the group comparison. Combined effect sizes that differ reliably from 0 are bold. $\mathrm{CI}=$ confidence interval.

$\dagger p<.10$ (marginally significant). $* p<.05 . \quad * * p<.01 . \quad * * * p<.001$. 
Table 15

Within-U.S. Meta-Analyses: Collectivism Sample Composition Moderator Analyses

\begin{tabular}{lcrccc}
\hline \multicolumn{1}{c}{ Group } & $\begin{array}{c}\text { Between-class } \\
\text { effect }\left(Q_{\mathrm{B}}\right)\end{array}$ & \multicolumn{1}{c}{$\begin{array}{c}\text { Mean weighted } \\
\text { effect size }\left(d_{i+}\right)\end{array}$} & $95 \% \mathrm{CI}$ & $\begin{array}{c}\text { Homogeneity within } \\
\text { class }\left(Q_{\mathrm{wi}}\right)\end{array}$ \\
\hline African Americans & 0.99 & & & & \\
$\quad$ Students & & 18 & -0.03 & $-0.12 / 0.08$ & $35.56^{* * *}$ \\
$\quad \begin{array}{l}\text { Nonstudent adults } \\
\text { Asian Americans }\end{array}$ & 1.04 & 2 & -0.21 & $-0.55 / 0.14$ & 1.73 \\
$\quad \begin{array}{l}\text { Students } \\
\quad \text { Nonstudent adults }\end{array}$ & & 29 & $\mathbf{- 0 . 4 0}$ & $-0.46 /-0.33$ & $73.19^{* * *}$ \\
Latino Americans & $1.16 \dagger$ & 2 & -0.20 & $-0.57 / 0.18$ & 1.08 \\
$\quad$ Students & & & & \\
$\quad$ Nonstudent adults & & 21 & $\mathbf{- 0 . 2 0}$ & $-0.30 /-0.09$ & $34.14^{*}$ \\
\hline
\end{tabular}

Note. Positive values indicate higher European American individualism; negative values indicate lower European American individualism. Studies with Cronbach's $\alpha<.70$ were classified as low reliability; studies with Cronbach's $\alpha \geq .70$ were classified as high reliability. For the Asian American comparisons, three studies were omitted because they lacked reliability information. Combined effect sizes that differ reliably from 0 are bold. $\mathrm{CI}=$ confidence interval.

$\dagger p<.10$ (marginally significant). $* p<.05$. *** $p<.01$. *** $p<.001$.

Our ability to make generalizations on the basis of the current body of empirical research is limited by significant within-group heterogeneity in regional, country, and ethnic group comparisons. Our next level of analyses examined possible moderation effects of scale reliability and content and sample composition (where possible given the nature of the database). Because most countries were represented by a single sample, more in-depth analyses were limited to the smaller subset of countries represented by multiple samples. Scale reliability varied, but analyses showed that it was not a sufficient explanation of heterogeneous effects, perhaps because of differences in scale content. With regard to content, the overall effects were either as expected or smaller than expected. Although data are not amenable to conclusive analyses, our interpretation is that for IND, valuing personal independence and personal uniqueness are core elements and for COL, sense of duty or obligation to in-group is the core element. Other values, though plausibly associated with these worldviews, are multiply determined and not necessary consequences of IND or COL worldviews. Because these other values (e.g., valuing self-knowledge

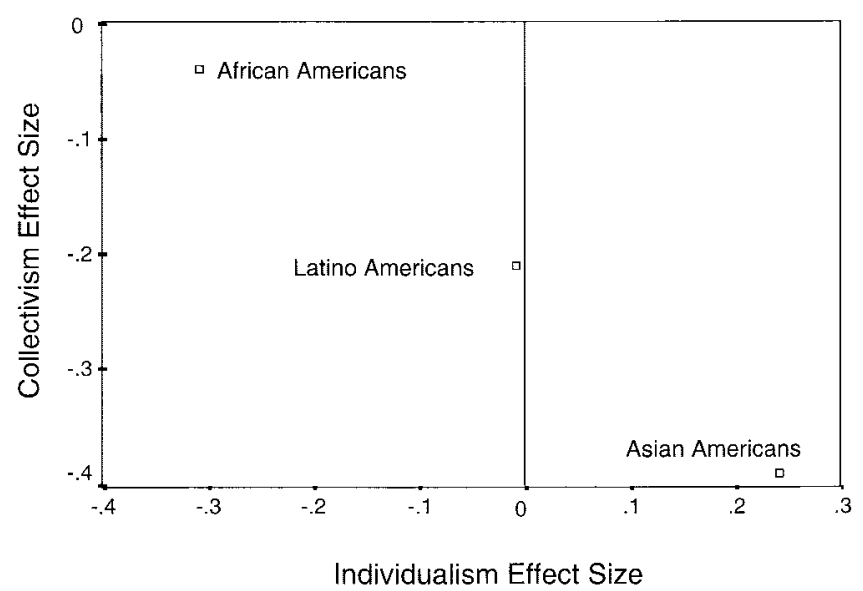

Figure 7. Within-U.S. meta-analyses: Plotting individualism and collectivism effect sizes for three ethnic groups. and seeking others' advice) did not provide stable results, we conclude that in the future, researchers should assess this content as separate constructs, when relevant to the particular research question under investigation.

Finally, we found evidence that the quality of relationality central to European Americans differs from that central to East Asians (in particular Hong Kong and Japanese participants). When feelings of belonging and enjoyment of group membership were included as items in measures of COL, European Americans scored higher than did East Asian participants. Conversely, when feelings of belonging to a group and enjoyment of group membership were not included as items in measures of COL, East Asians were higher or no different in COL than European Americans.

\section{REVIEW OF THE LITERATURE ON IMPLICATIONS OF IND AND COL FOR BASIC PSYCHOLOGICAL FUNCTIONING}

In this section, we review findings from studies focused on the implications of cultural frame for self-concept, well-being, attribution, and relationality. In each of the following sections, we provide a brief summation of findings followed by a thumbnail sketch of the evidence on which we based our assessment.

\section{Overview}

The meta-analyses provided some evidence that overall, European Americans are different in IND and COL from others. The meta-analyses also supported the notion that cultural differences in IND focus on valuing personal independence and personal uniqueness (and sometimes personal privacy), whereas differences in COL focus on group processes - valuing duty (and for international comparisons, in-group harmony). In this section, we focus on the second aspect of our basic aim, asking, Are theoretically derived implications of individualism and collectivism for psychological functioning borne out in the empirical literature?

Appendix C presents a summary of all studies reviewed in this section. Each study is listed by domain, noting the following: 
whether it included a within-U.S. or cross-cultural comparison (or both), sample size and origin, the composition of the sample (e.g., college student, administrator), measures used in the analyses reported here, the research method used, and basic findings, including a measure of effect size where possible. In our summary, we refer to the size of an effect as a global reference to the size of correlations or percentage difference found between groups. When possible, we translated presented statistics into an estimate of effect size following the same convention used in the metaanalyses to refer to small, moderate, and large effects. Using the equivalence equation $d=2 r / \sqrt{1-r^{2}}$, we translated reported correlations into effect sizes to be able to interpret them according to Cohen's (1988) guidelines. Accordingly, we refer to small effects as those with $r<.20$, moderate effects as those with $.20<r<.33$, and large effects as those with $r>.33$.

When possible, we extracted and computed statistical information pertaining to size of relevant effects. We consider the derived effect size to be a thumbnail estimate based on the available evidence. However, the research reports were extremely heterogeneous both in method and in type of information supplied. For reports that did not provide sufficient information to calculate an effect size, Appendix C provides a brief narrative summary of central findings. Otherwise, we report a variety of different quantifiable effect size measures (e.g., $d, r, \tau)$ or difference in percentage points, depending on the nature of the information provided.

Given our efforts to quantify results, the reader may wonder whether we use a meta-analytic approach in this section as well; however, we felt that it would be inappropriate to summarize the diverse research bearing on this topic meta-analytically. This research covers a broad spectrum of topics and is diverse as to method and measures. For example, in the self-concept domain, there is a great degree of heterogeneity among studies in both research questions and research approaches. Except for a number of studies that used the Twenty Statements Test (Kuhn \& McPartland, 1954), no two studies used the same dependent measure. Second, comparison countries or groups varied widely between studies (e.g., Nigeria-U.S. and PR China-U.S.); synthesizing across samples with meta-analyses would ignore these theoretically important differences.

Therefore, in this section we use a qualitative approach to examine the extent that differences in IND and COL have consequences for basic psychological functioning in the domains of self-concept, well-being, attribution, and relationality (including studies using workplace as the basis for study of relationships). We distinguish research that assumes that Americans are higher in IND and lower in COL than others from research that actually assesses IND and COL. We also highlight methodology, noting studies that used experimental rather than correlational techniques, because the former are better able to illuminate the process by which IND and COL influence psychological functioning. Finally, we distinguish research that used within-United States samples from cross-national research and note whether the sample was composed of participants other than college students.

\section{Self-Concept and Self-Esteem}

\section{Overview}

Recall that the theoretical literature proposed that IND is associated with concern for maintaining and enhancing self-esteem and defining the self through unique traits rather than social roles, whereas COL is associated with diminished centrality of selfconcept and viewing the self as part of larger social groups and endeavors. What is the empirical evidence that European Americans differ from others in these ways? The research reviewed in this section can be summarized as such: When country is used as a stand-in for cultural frame, an argument can be made that IND is associated with more optimism or higher self-esteem whereas COL is associated with a more interpersonal and social selfconcept. Effect sizes for self-concept differences are variable, and large effects occur, especially when the researcher examined collective- or in-group-focused content and directly assessed INDCOL. For self-esteem, although effects are moderate to large, their relation to IND-COL is indirect both because of the emphasis on comparisons with Japan, a country found to be lower in COL in the meta-analyses, and because of a lack of explicit assessment of IND-COL.

As seen in the first section of Appendix C, we found 30 relevant studies, 3 focused on personality correlates of IND and COL, 15 focused on content of self-concept, and 12 focused on self-esteem. All but 2 of these studies compared European American undergraduates with other undergraduates, and one third of this research compared American subgroups only. Japan was the primary crossnational comparison for self-esteem research. In two thirds of this research, researchers did not assess IND-COL; rather, they assumed European Americans were higher in IND and lower in COL compared with others. Direct assessment or manipulation of IND and COL occurred in only 11 studies. Personality and self-concept research was correlational, mostly focused on between-group comparisons, whereas half of the self-esteem studies involved experimental manipulations.

\section{Personality Traits}

Three studies (two within-U.S., one comparing Americans to Japanese and Koreans) focused on personality traits and attempted to find associations with assessed IND and COL. Indeed, COL, assessed as interdependence or allocentrism, correlated with congruent personality traits in Americans, Japanese, and Koreans. Specifically, when COL was assessed as a focus on the peer group, it correlated positively with need for affiliation and sensitivity to rejection and negatively with need for uniqueness among undergraduates from Korea, Japan, and the United States (Yamaguchi, Kuhlman, \& Sugimori, 1995). Similarly, interdependence correlated with need for affiliation, nurturance, succorance, and abasement among European American undergraduates (Hui \& Villareal, 1989, Study 2) and Asian Canadian and European Canadian students high in family-focused COL were more likely to describe themselves as meeting expectations of close others (Lay et al., 1998, Study 2). Results within the United States and Canada are thus congruent with data including Japanese and Koreans, suggesting that COL functions the same way in its impact on personal traits across these groups. Observed effects were large but included a small number of studies.

\section{Content of Self-Concept}

Fifteen studies examined content of self-concept using correlational research methods. Eight studies compared groups assumed 
to differ in IND-COL, and seven studies measured IND-COL explicitly. In eight studies, respondents described themselves using the Twenty Statements Test or other open-ended self-description tasks. Typically, these responses were content coded, distinguishing traits from social descriptors. European American undergraduates used more personal trait descriptors (and sometimes fewer social role descriptors) than Asian Americans, Chinese, Indians, Kenyans, and Koreans (Dhawan, Roseman, Naidu, Thapa, \& Rettek, 1995; Ma \& Schoeneman, 1997; Rhee, Uleman, Lee, \& Roman, 1995; Trafimow et al., 1991). Junior high school European American students rated individualistic self-descriptors as more important than collectivist self-descriptors and used fewer groupfocused self-descriptors than did Mexican American students (Dabul, Bernal, \& Knight, 1995). European American undergraduates high in COL, assessed as interdependence, used a greater number of social identities to describe themselves than did European American undergraduates low in COL (Gaertner, Sedikides, \& Graetz, 1999, Study 4). European Canadian undergraduates higher in COL, assessed as familialism, rated their ethnic backgrounds as more self-defining (Lay et al., 1998, Study 3). Among European, Korean, and Chinese Americans, IND correlated more strongly with personal identity and COL correlated with social identity (Wink, 1997). Similarly, Americans higher in COL were higher in in-group pride (J. W. Jackson \& Smith, 1999, Studies 1 and 2).

However, as seen in Appendix C, effects were variable and not entirely consistent. Thus, for European American and Asian American undergraduates, higher IND correlated with use of more personal- and more family-focused self-descriptors (C. A. Johnson, Southwick, Carlson, \& Hsai, 1997). Hong Kong Chinese and Japanese students used more personal preference-focused descriptors than did European American students (Cousins, 1989; Ip \& Bond, 1995), and Hong Kong Chinese, Japanese, and European American students were equally likely to use social descriptors, although Japanese used fewer family descriptors than either Americans or Chinese (M. H. Bond \& Cheung, 1983). Moreover, in a study correlating collective self-esteem with IND and COL, only IND had a positive association with importance of group membership for Canadian and Japanese students, and for Japanese students, private valuation of one's group was equally correlated with both IND and COL (Sato \& Cameron, 1999). Taken together, the open-ended self-concept studies that constitute almost $75 \%$ of this section provided only weak support for the notion that European Americans differ from others in content of self-concept because direct assessment of IND-COL was rare and content coding of responses differed from one study to the next. Therefore, it is often impossible to say with any certainty whether IND or COL was the mechanism behind the observed differences.

\section{Self-Esteem}

Twelve studies, most of which were cross-national, focused on self-esteem. This area included experimental as well as correlational and group comparison methods, dominated by comparisons with Japan. Taken together, they suggest that different forces operate in the function and maintenance of self-esteem for Japanese as compared with Americans (and Canadians). Effect sizes were moderate to large. When assessed as independence and interdependence, higher IND correlated with higher self-esteem in
Japanese and American (and Canadian) undergraduates (Carter \& Dinnel, 1997; Hetts, Sakuma, \& Pelham, 1999), but higher COL correlated with lower self-esteem in only one of these studies (Hetts et al., 1999). When primed as self versus group focus, IND was associated with viewing the self more positively among European Americans and Asian Americans but not among recent Asian migrants (Hetts et al., 1999, Studies 1 and 2).

In terms of self-esteem function and maintenance, European American undergraduates rated success situations as both more relevant to self-esteem than failure situations (Kitayama et al., 1997) and as more self-defining (Tafarodi \& Swann, 1996), whereas Japanese undergraduates rated failure situations as more relevant (Kitayama et al., 1997). In the same manner, European Canadians viewed IND-relevant negative life events as less likely, whereas Japanese viewed COL-relevant negative life events as more likely, to occur to them than did their peers (Heine \& Lehman, 1995, Study 2). In addition, experiencing success motivated European Canadian undergraduates more than Japanese (Heine, Kitayama, Lehman, Takata, \& Ide, 1998). European Canadian undergraduates were more optimistic (Heine \& Lehman, 1995, Study 1) and less likely to mistakenly believe their performance was below average when they received success feedback (Heine, Takata, \& Lehman, 2000).

Finally, undergraduates in countries that Hofstede (1980) reported to be higher in IND rated personal success as more important for self-esteem, whereas undergraduates in countries Hofstede rated as lower in IND rated family life as more important for self-esteem (Watkins et al., 1998). ${ }^{5}$ Self-esteem contributed more to the life satisfaction of U.S. undergraduates than to the life satisfaction of Hong Kong undergraduates. Likewise, relationship harmony contributed more to the satisfaction of Hong Kong undergraduates than to the life satisfaction of U.S. undergraduates (Kwan, Bond, \& Singelis, 1997). However, Chinese were higher in self-liking than European American students, whereas European Americans were higher in self-competence (Tafarodi \& Swann, 1996).

\section{Summary}

One of the central claims of cultural psychology is that INDCOL cultural frames set up characteristic ways of making sense of the self, and, indeed, 30 studies examined aspects of this claim. This area of research shows promise; COL does make salient social, collective, and related aspects of the self-concept, at least under some measurement conditions. Limitations due to focus on correlational methods and emphasis on cross-national comparisons with Japan notwithstanding, next steps for researchers in this area require clarifying process issues. An open question is whether cultural differences in IND and COL have the most influence by affecting what is chronically salient about one's self-concept or by affecting how the self-concept is structured. Moreover, it is not yet clear that a cultural frame's influence on motivation, well-being, and attribution style occurs through its influence on self-concept

\footnotetext{
${ }^{5}$ Hofstede rated five countries as high IND (Australia, Canada, New Zealand, United States, and White South Africans); the remaining 10 were either rated low IND by Hofstede or assumed low IND by the authors (China, Ethiopia, Hong Kong, India, Malaysia, Nepal, Nigeria, Philippines, Zimbabwe, and Black South Africans).
} 
(see Matsumoto, 1999; Takano \& Osaka, 1999, for a similar critique of this area).

\section{Well-Being and Emotion}

\section{Overview}

Recall that the theoretical literature links IND and COL with different sources of well-being. Attaining personal goals, happiness, and personal control are assumed central to well-being within IND, and carrying out obligations and duties are assumed central to well-being within COL. With regard to emotional expression, the theoretical literature is less clear. We speculate that COL might plausibly carry with it sensitivity to decoding interpersonal emotions of others rather than valuation of expressing positive, selffocused emotions and make explicit taboo the transmission of some emotions (such as anger at in-group members). Because collectivists will therefore make efforts to control emotional expression, collectivists may be less likely to express emotion through facial expression and more likely to express emotion more indirectly. Conversely, IND might carry with it valuation of emotional expression and focus on the face as the seat of emotional expression. What is the evidence that European Americans differ from others in well-being and emotional expression?

Research reviewed in this section can be summarized as follows: Hofstede's (1980) IND ratings for various countries tend to moderate the correlations between sources of satisfaction and general life satisfaction. However, IND has an effect primarily in research that does not control for country differences on other variables. Research that used these controls showed smaller effect sizes attributable to IND. Furthermore, as the number of possibly confounding variables controlled increases, the impact of IND decreases. Assessed IND correlates with lower social anxiety and reduced vulnerability to depression but only for non-Asians, presumably because IND is less socially valued in Asian societies. In terms of emotional expression, effect sizes for self-reported embarrassability tended to be large and positively associated with COL. Effect sizes for emotional expression were also generally large and positively associated with IND. Other emotion-related analyses did not provide enough information to calculate effect sizes.

We found 29 correlational studies that examined well-being and emotions and their relationship with IND and COL. Nineteen studies focused on well-being, 9 of which used overlapping data sets from multinational surveys, allowing for tighter comparisons than in the previous section. This research did not include a direct assessment of IND or COL at the individual level. However, 10 additional studies that did examine the direct relation between IND and/or COL and aspects of well-being were found, although only 2 of these latter studies were cross-national. With regard to emotions and emotional expression, 10 studies, 3 assessing IND-COL directly, were found. Six of these studies used cross-national comparisons, 1 involved multinational comparisons, and 3 used withinU.S. samples.

\section{Well-Being}

We found 19 relevant studies, all correlational. Eight multinational studies correlated average levels of well-being of individuals within a nation, using a single-item well-being variable, with Hofstede's nation-level ratings of IND (supplemented in Diener's research with nation-level ratings of IND provided by Triandis [Diener \& Diener, 1995; Diener et al., 1995; Gohm, Oishi, Darlington, \& Diener, 1998; Oishi, Diener, Lucas, \& Suh, 1999; Suh, Diener, Oishi, \& Triandis, 1998]). Data from Michalos (1991) and Veenhoven (1993), who collected student data in 42 countries, and the World Values Survey Group (1994), with data from more than 55,000 men and women from 41 nations, were used in all of these studies. Findings varied depending on the controls introduced. When national wealth, civil rights, and social comparison of income were controlled, no relation between IND and wellbeing was found (Arrindell et al., 1997). Moreover, cluster and structural equation analyses failed to find a moderating effect of IND on the relation between satisfaction with specific domains assumed more central to IND (personal achievement) or COL (family) and general life satisfaction (Mallard, Lance, \& Michalos, 1997).

Without these controls, life satisfaction correlated with feeling more positive than negative emotions (Suh et al., 1998, Studies 1 and 2) and correlated more positively with the self- and friendshipsatisfaction items in high-IND than low-IND countries (Diener \& Diener, 1995). Similarly, marital status and quality were more closely related to well-being in high-IND compared with low-IND countries (Gohm et al., 1998). When either income or social equality was controlled, IND correlated with life satisfaction (Diener, Diener, \& Diener, 1995). Another study found that the extent to which satisfaction with self and satisfaction with freedom predicted a person's level of life satisfaction was a function of IND: When cultural IND was high, satisfaction with self and satisfaction with freedom were more influential in predicting one's overall well-being (Oishi et al., 1999, Study 2).

Another group of studies using data from the World Values Survey and four representative U.S. samples suggested a more specific relation between well-being and IND-COL when socioeconomic status, household status, sociodemographics, and gross domestic product were controlled. With this data set, Sastry and Ross (1998) showed that the relationship between perceived personal control (plausibly more valued in IND cultures) and feelings of depression (well-being) varied for Asians and non-Asians. Specifically, increased control was associated with less depression for European Americans and African Americans, whereas for Asians and Asian Americans personal control was unrelated to depression, presumably because personal control is less culturally valued. Sastry and Ross obtained matching results when comparing samples from Asian countries with those from non-Asian countries. ${ }^{6}$ Congruently, in a sample of European and Asian Americans, anxiety and depression correlated negatively with independent self-construals and positively with interdependent self-construals (Okazaki, 1997, 2000). Sato and McCann (1998) obtained similar findings for depression in a Canadian sample. Two crossnational studies with Japanese and European American undergraduates replicated the negative relation between social anxiety and independent self-construals (Dinnel \& Kleinknecht, 1999; Kleinknecht, Dinnel, Kleinknecht, Hiruma, \& Harada, 1997). Both

\footnotetext{
${ }^{6}$ The authors weighted the World Values Survey for underrepresentation of rural areas and overrepresentation of smaller countries.
} 
studies also replicated the positive correlation between interdependent self-construals and social anxiety for European Americans. For Japanese, Dinnel and Kleinknecht (1999), but not Kleinknecht et al. (1997), found a significant positive correlation between COL and social anxiety.

In research assessing IND and COL among American undergraduates, well-being was negatively correlated with IND and positively correlated with COL for European Americans, but this relation disappeared when racial identity was controlled (Bettencourt \& Dorr, 1997, Studies 1 and 2). Similarly, COL positively correlated with psychological adjustment among European Americans but not among minority individuals or when levels of family support and acculturation were controlled (Ebreo, 1998). COL also moderated the relation between experiencing daily hassles and depression among Vietnamese Canadian adults (Lay et al., 1998, Study 4), again suggesting a potentially positive impact of COL on well-being among Americans (and Canadians).

\section{Emotions and Emotional Expression}

Ten studies examined sources of happiness and appropriateness of expressing happiness and embarrassability in American, Chinese, Costa Rican, El Salvadoran, Hong Kong, Japanese, and Russian undergraduates. Effect sizes, where calculable, were small. European American undergraduates reported that a brother's transgressions and a child's accomplishments were less relevant to their own happiness compared with Chinese undergraduates (Stipek, 1998). Americans rated happiness as more common, and they believed attaining happiness was a more important, desirable, and attainable goal than did Russians (Lyubomirsky, 1997). Americans also perceived happiness as a more appropriate emotion to express than did Japanese (Matsumoto, 1990). However, compared with Americans, Russians said that they were more likely to express their happiness to everyone (Lyubomirsky, 1997). Similarly, $34 \%$ of El Salvadorans said that pursuing and reaching valued goals was a source of happiness but only $21 \%$ of Americans said this (Chiasson, Dube, \& Blondin, 1996). Multiethnic undergraduates in New Mexico reported feeling more comfortable expressing emotions, even interdependent emotions (e.g., feeling connected to family members), than did Costa Rican or Japanese students (C. W. Stephan, Stephan, Saito, \& Barnett, 1998; W. G. Stephan, Stephan, \& de Vargas, 1996). Given that none of these studies assessed IND-COL, it is unclear to what extent they bear on IND-COL, even though the studies' authors tend to believe they do.

Three studies explored the association between the social emotion of embarrassment and either IND or COL. In all three studies, sensitivity to embarrassment was negatively correlated with IND and positively correlated with COL. Samples included Asian American and European American undergraduates (Sharkey \& Singelis, 1995; Singelis \& Sharkey, 1995) and undergraduates from Hong Kong, Hawaii, and the U.S. mainland (Singelis, Bond, Sharkey, \& Lai, 1999).

Finally, a multinational study by Scherer (1997) drew on data from 37 countries, examining appraisals of different emotioneliciting situations. Between-country differences in appraisals were small and not related to Hofstede (1980) IND scores.

\section{Summary}

Hofstede's (1980) data link IND with national wealth, GNP, and other factors related to modernization. All of these factors are also likely to increase life satisfaction and well-being, making analyses of the additional influence of IND and COL on well-being difficult. To show an effect, researchers need to disentangle the effect of IND and/or COL from other country-level differences that relate to life satisfaction or show that IND and COL make salient different bases of life satisfaction.

Congruent with Hofstede's (1980) earlier results, the multinational well-being studies reviewed in this section show that although well-being and Hofstede's initial IND scores (sometimes supplemented with Triandis-rated IND) were moderately correlated, this relation was at least partly mediated by national wealth and civil rights factors. Although it is not yet clear whether IND and COL shape the relation between satisfaction in life domains and well-being, progress has been made in examining the influence of personal control on well-being versus depression. Low IND, high COL, and lack of personal control all correlate with vulnerability to depression in European Americans. The link between IND-COL and emotional expression or appraisal is consistent only for embarrassability - a highly socially contextualized emotion assumed to be associated with lower IND and higher COL. Unfortunately, cross-cultural research in emotions has not yet provided a clear empirical basis for linking IND or COL with emotional expression.

\section{Attribution Style}

\section{Overview}

Recall that the theoretical literature implicates IND with personfocused and decontextualized causal reasoning and COL with contextualized and situated reasoning. What is the evidence that European Americans differ from others in these ways? The empirical research reviewed below can be summarized as such: European Americans use an individualistic processing style and find relational and contextual information less informative or compelling than others, even when contextual influences are made salient. Americans' thinking about social obligation is qualified by the nature of individual need and the type of relationship to the other. Obligation research is limited by its focus on contrasts with one country (India) and lack of assessment of IND-COL.

As seen in the third section of Appendix C, 40 studies examined cultural and cross-cultural aspects of attribution style, with 29 focusing on explanations, 6 on persuasion, and 5 on attributions about obligations. As is typical, most studies in this section involved undergraduate participants (18 explanation studies, 5 persuasion studies, and all obligation studies). However, this area also contains a sizable number of studies including both children and nonstudent adults. All within-United States studies focus on attributions about explanations. Cross-national studies involved primarily Indians, Chinese, and Koreans. The research in this area provides a strong basis for conclusions about the effects of IND and COL on attribution because attribution researchers tend to use experimental methods, assess IND and/or COL, use diverse participants (not only students), and do not focus heavily on comparisons with Japan. 


\section{Explanations}

We found 29 studies focusing on social explanation processes. A sizable majority (21 studies) used experimental manipulations; only 4 studies both were correlational and did not assess INDCOL. Overall, effects were moderate to large.

Following the assumption that American individualism carries with it dispositional causality, American undergraduates used more internal attributions than did Saudi students (Al-Zahrani \& Kaplowitz, 1993). Asked to explain two shootings, American graduate students were more likely to make dispositional attributions, whereas Chinese graduate students were more likely to attribute behavior to situational circumstances (Morris \& Peng, 1994, Study 3). Similarly, the sports pages of U.S. newspapers exhibited a greater tendency toward dispositionalism than the sports page of newspapers in Hong Kong (F. Lee et al., 1996). Consistent results were also found in a series of studies with Hindus: American adults explained behavior and outcomes more in terms of dispositions, whereas Hindus focused more on situations in describing the behavior of both others (Shweder \& Bourne, 1982) and themselves (Miller, 1984). For Hindus this was true whether the behavior was deviant or prosocial (Miller, 1986), with focus on situational circumstances increasing with age. Further, American undergraduate women were more likely to use their personal moral code versus social standards to explain norm violations than were Hindu undergraduate women (Verma, 1986). Notably, Hindus, but not Americans, viewed reciprocating help as a moral obligation (Miller \& Bersoff, 1994) and saw fulfilling social role obligations as moral obligations, not personal choices (Miller \& Luthar, 1989). Last, Hindu adults and children were more likely to take into account emotional distress in absolving agents of accountability for lapses in appropriate behavior resulting in injustice (Bersoff \& Miller, 1993).

Cultural differences in attribution became even more apparent when contextual information was salient. Although in a control condition Korean and American students were equally likely to use dispositional information, differences emerged in their use of contextual information only when situational constraints were salient (Choi \& Nisbett, 1998). In addition, for American high school (Morris \& Peng, 1994, Study 1) and graduate students (Morris \& Peng, 1994, Study 2), dispositional reasoning was more salient in social than physical reasoning tasks, particularly when the social reasoning tasks focused on individuals rather than groups. American students emphasized dispositions for individuals and situations for groups, whereas Hong Kong Chinese emphasized dispositions for groups and situations for individuals (Menon, Morris, Chiu, \& Hong, 1999, Study 2), although Hong Kong Chinese were higher in situational attributions overall (Menon et al., 1999, Study 3).

All of the research reviewed in this section so far has involved use of comparison groups rather than assessment of IND and/or COL to make the point that European American attribution style focuses more on dispositional and decontextualized reasoning and less on situated reasoning than is true for other groups. Five studies assessed IND and/or COL and three studies primed independence versus interdependence to make a more direct link between attribution style and cultural frame. Four of these studies found the posited connection between trait- rather than situation-focused reasoning style and assessed IND. Specifically, among Americans,
IND correlated with increased use of trait-based inference and decreased use of situation-cued recall (Duff \& Newman, 1997, Studies 1 and 2; Newman, 1993, Studies 1 and 2), although trait-based inferences were more prevalent than situation-based inferences overall. Four studies included U.S. students from a variety of backgrounds and showed a significant positive association between interdependence (not independence) and use of contextual information to draw inferences (Singelis \& Brown, 1995). This finding was replicated in three priming studies (Ybarra \& Trafimow, 1998, Studies 1-3), in which participants primed with interdependence based their attitudes more on the expectations of others, whereas those primed with independence based their attitudes more on personal preference.

Moreover, attribution research shows that when explaining and predicting social behavior, European Americans focus more heavily on dispositions than do others - even in cases where our meta-analysis did not show any IND or COL difference between European Americans and these others. For example, even though we did not obtain any IND differences between European Americans and Latinos in our meta-analyses, European American children expected others to behave in trait-consistent ways more than did Latino children, who were more sensitive to context (Newman, 1991, Studies 1 and 2). Similarly, American children believed more strongly that they could control their own personal outcomes through effort than did German and Russian children (Little, Oettingen, Stetsenko, \& Baltes, 1995). Taken together, though the relation to the IND-COL framework is unclear, research strongly suggests that dispositional reasoning is an American attribution style and that among Americans, those higher in independence are more dispositional, whereas those with more interdependent views are more situated in their reasoning.

Only two research reports failed to support these conclusions. First, a correlational analysis using American and Brazilian participants did not find a correlation between IND and use of social norms versus personal feelings to decide how to behave (Bontempo, Lobel, \& Triandis, 1990). Second, Krull et al. (1999, Studies 1 and 2) did not find any evidence that Chinese are less susceptible to privileging personal dispositional explanations over social contextual explanations for behavior. That is, U.S., Taiwanese, and PR Chinese participants were equally likely to believe that an essay's content reflected the author's genuine beliefs when they assumed that the essay was the author's free choice and when the experiment made clear that the author was instructed to write this particular essay-and their attributions were not correlated with IND or COL.

Our meta-analyses suggest that Brazilians may be higher in IND and not much different in COL than Americans, which may be a partial explanation for the Bontempo et al. (1990) results. However, the Krull et al. (1999) results show neither country-based effects of IND-COL nor individual-based effects of allocentrismidiocentrism and therefore deviate markedly from all other attribution research reviewed in this section.

\section{Persuasion}

Four of the six studies reviewed in this section included an international comparison and involved experimental methods, whereas two used correlational methods with U.S. samples. 
With regard to persuasive appeals, American adults found individualistic advertising appeals more compelling than collectivist appeals, with the reverse being true for Koreans (Han \& Shavitt, 1994, Study 2). In addition, compared with Polish undergraduates, American undergraduates were more likely to be persuaded to participate in a survey if their own past cooperation was made salient than if their group's cooperation was made salient (Cialdini, Wosinska, Barrett, Butner, \& Gornik-Durose, 1999), but an equivalent effect was not replicated in a comparison with a Mexican sample (Wosinska et al., 1999). Assessed levels of IND and COL moderated this effect. Both American and Polish undergraduates high in IND were more persuaded by individualistic appeals to their own past cooperation, whereas undergraduates high in COL were more persuaded by collectivistic appeals to their group. Further, in uncertain social situations, American students found individuating information more useful than relational information, with the reverse being true for PR Chinese students (Gelfand, Spurlock, Sniezak, \& Shao, 2000). Similarly, American college students (Kemmelmeier, Burnstein, \& Peng, 1999) and adults (Kemmelmeier et al., in press, Study 4) high in IND perceived physician-assisted suicide, framed in terms of personal freedom and self-determination, more favorably than did low-IND Americans.

\section{Obligations}

Of the five studies in this section, three examined the relation between obligation and IND-COL in U.S. samples. Two studies with Jewish- and Asian American undergraduates (Oyserman et al., 1998, Studies 1 and 2) manipulated salience of cultural frame (IND and COL), target of obligation (in-group vs. larger society, individual vs. group), and frame of reference (personal goals presented first vs. request for help presented first). Participants rated whether they would help, and analyses focused on response, response latency, and confidence judgments. Overall, COL increased obligation, especially when salient. Participants high in both IND and COL were more obligated to individuals and larger society. A third experiment by Steelman (1995) found that among White and minority American participants high in IND, affective response moderated willingness to help and even perception of need for help. High-IND individuals helped only targets that elicited pity or a similar affective response, whereas affect did not moderate the responses of high-COL participants.

Two studies contrasted Hindu and American adults and children without assessing IND or COL. Findings were similar to the findings for high-COL Americans reported earlier. For Hindus, obligation was independent of attributions of closeness, liking, or need. Hindu and American adults were equally likely to help in cases of extreme need or when the request came from parents (whether their need was moderate or extreme). The distinction came for minor needs or requests from friends and strangers; in these cases, Hindu, as compared with American, adults and children continued to feel highly obligated (Miller, Bersoff, \& Harwood, 1990, Study 1). Similarly, for Americans greater liking led to greater sense of obligation; for Indians liking was inconsequential to obligation (Miller \& Bersoff, 1998). Americans considered extenuating circumstances when deciding whether they should feel obligated; Indians did not. For the latter group, obligation seems more of a simple imperative.

\section{Summary}

We construed attribution style broadly to include the nature of explanations, differences in the effectiveness of persuasive appeals, and differences in thinking about social obligation. In combination, this research provides remarkably consistent evidence that Americans are more likely to focus on dispositions in providing rationales for behavior or explaining causality. Where measured, it appears that differences in IND and COL predict differences in attribution. Effect sizes, where available, tend to be moderate or even large. The small literature on persuasion and the emerging literature on social obligation also highlight the differences in what is persuasive and what obligation looks and feels like. Two of four persuasion studies provide too little data to calculate effect sizes; nonetheless, Americans see individual-focused appeals as more persuasive than Koreans, Poles, Mexicans, and PR Chinese, who find collective-focused appeals more persuasive. Similarly, Americans are more likely to qualify their obligation to others by level of need, reason for need, and the nature of their relationships with others than Hindu Indians do. Within the United States, differences in IND and COL produce the same effects, and when both are measured, each has an independent effect on the nature of obligation. This orthogonality of the effects of IND and COL mirrors the orthogonality of country and group levels of IND and COL found in the meta-analyses.

\section{Relationality and Groups}

\section{Overview}

Recall that the theoretical literature proposes that IND leads to ambivalence concerning close relationships and fosters a willingness to leave relationships that are not beneficial to the person. Conversely, the theoretical literature implicates COL with use of equality norms and willingness to remain permanently in relationships, even in personally costly ones. What is the evidence that European Americans differ from others in these ways? Perhaps because of the centrality of relationality-group relations to the IND-COL distinction, more studies focused on this than on any other reviewed topic (71 studies). ${ }^{7}$ Yet, the extremely diverse research related to this broad topic does not lend itself to simple summary. Therefore, we divided studies into three main areas, each including two or three subtopics: (a) close relationships (7 family and 10 intimate relationship studies), (b) in-group and out-group interactions (12 social behavior, 10 communication style, and 16 conflict resolution style studies), and (c) work or organizational contexts ( 8 working in groups and 8 conflict management studies).

Broadly speaking, the empirical research reviewed below indicates that IND and COL matter for relationality and group relations. Although the research does not support the notion that Americans have conflicted family and intimate relations, it does support the idea that IND promotes ease of interacting with strangers and more direct communication styles and that COL promotes

\footnotetext{
${ }^{7}$ We dropped a potential 72nd study (Fijneman, Willemsen, \& Poortinga, 1996) because we did not know how to interpret whether results were cross-national or a measurement artifact.
} 
in-group preference in relationships and different forms of facesaving. Effect sizes are often moderate to large, though highly variable. Intimacy research is fragmented; for closeness, when IND-COL is measured, samples involve only Americans. As we review below, effects are small for differences in conflict resolution style and large for working in groups. Effects for conflict management are heterogeneous, though very large for a single multinational study.

Work and organizational research allows for stronger conclusions than close relationship and in-group/out-group relations studies. Most work-related research directly assessed IND-COL (over $80 \%$ of the studies), but IND-COL measurement was less frequent in close relationship (just over 50\%) and in-group/out-group relations studies (under 40\%). Furthermore, experimental design was more common in work or organizational research (50\% of studies) than in close relationship $(0 \%)$ and in-group/out-group relations studies (about 30\%). Overall, research in this area was primarily cross-cultural-less than a quarter of the research base includes only American respondents.

\section{Close Relationships}

\section{Family Obligation}

Several studies assess COL as family obligation and found Americans to be higher in family focus than Chinese and Japanese but lower than Koreans (Hui, 1988, Study 1; Matsumoto et al., 1997; Rhee et al., 1996). This result echoes the results of our meta-analyses, which suggest that family focus is separate from COL. Closer examination of these studies suggests a different conceptualization of obligation among European Americans compared with others. For example, European American and Mexican American students did not differ with regard to degree of obligation to family, but European Americans equated obligation to family with relationship quality and closeness to family members and therefore viewed obligation to family as a personal choice. For Mexican Americans, obligation related to familialism, and collectivism was more a part of the social role of being a family or group member (Freeberg \& Stein, 1996). In addition, although European Canadian and Japanese Canadian students rated relationships with family, friends, and romantic partners as closer than did Japanese students, all participants rated their own relationships with family and friends as closer than the relationships of their peers (Endo, Heine, \& Lehman, 2000, Studies 1 and 2).

Wink, Gao, Jones, and Chao's (1997) comparison of Chinese American and European American undergraduates underscores the complexity of disentangling the quality of family relationships from IND and COL, especially when these latter constructs are not measured. Chinese American and European American students did not differ in the extent that they defined themselves as separate from their parents, but European American undergraduates saw their parents as more respectful of their independence than did Chinese American students. European Americans not only felt more supported in their independence, they also rated their relationship with their parents as more emotionally supportive and more mutual and felt more comfortable asking their parents for support than did Chinese American undergraduates.

\section{Relationship Closeness}

Ten studies explored relationship closeness, centrality of romantic love, intimacy, and ambivalence in relationships, finding some support for lower relationship commitment and intimacy among Americans but mixed results with regard to romantic love. First, among European American students, higher IND correlated with lower relationship commitment (Agnew \& Lee, 1997; Kemmelmeier, Sanchez-Burks, Cytron, \& Coon, 1998, Study 2). Second, American students both expressed more relational ambivalence than Japanese students and rated their relationships as more openly conflictual (Ting-Toomey, 1991). Third, Canadian students who rated themselves high in personal autonomy were less likely to report ever having been in love or having intense feelings of love and were lower in trust, need for, attraction to, and caring for a romantic partner (Dion \& Dion, 1991). Yet, American students were more satisfied with their dating relationships than were Chinese students (Lin \& Rusbult, 1995).

Next, in a multinational survey by Levine, Sato, Hashimoto, and Verma (1995), Hofstede's (1980) IND rankings correlated positively with viewing lack of romantic love as a barrier to marriage, with Americans scoring highest in endorsement of lack of romantic love as a reason not to marry. However, Americans ranked only ninth of 11 countries in their belief that lack of romantic love is a good reason to dissolve a marriage. Moreover, American belief in romantic love was no higher than that of West German or Japanese students (Simmons, vom Kolke, \& Shimizu, 1986), and European American graduate students in Hawaii were no more likely to be in love than native Hawaiians (Doherty, Hatfield, Thompson, \& Choo, 1994). Furthermore, both IND and COL correlated with extent of self-disclosure in marriage for Chinese from the People's Republic, Chinese nationals in the United States, and U.S. couples (Liang, 1997). IND and COL related to different aspects of romantic love beliefs among European American students (Kemmelmeier et al., 1998, Study 1).

\section{Interactions With Others}

\section{Social Behavior}

Twelve studies focused on social behaviors, finding support for the notion that European Americans differ from others in their social behaviors and some evidence that this relates to differences in IND-COL. First, R. Bond and Smith (1996) conducted a metaanalysis of studies using the Asch line judgment task and showed level higher conformity in respondents from countries lower in IND according to Hofstede's (1980) individualism scores and Schwartz's (1994) value ratings. In addition, whereas Japanese and Hong Kong students reported spending more time with in-groups than out-groups, European Americans spent equal time with inand out-groups (Gudykunst et al., 1992) and reported having more freedom to decide which groups to belong to than Indians (Verma, 1985) or Asian Americans (Peng, Kemmelmeier, Burnstein, \& Manis, 1996). Americans also reported belonging to more groups than did Indian students, although they did not differ in how much they follow in-group advice (Verma, 1985); the latter finding is echoed in our meta-analyses.

Whereas American college students reported treating close friends, coworkers, or business owners similarly, Chinese (Hui, 
Triandis, \& Yee, 1991) and Brazilians (Pearson \& Stephan, 1998) did not. ${ }^{8}$ Although interacting with in-group members was always found easier than interacting with strangers, Japanese and Koreans reported greater disjuncture between the two situations than did American respondents (Gudykunst, Yoon, \& Nishida, 1987). Americans interacted with more people, more frequently, whether on a one-to-one basis or in small groups, than did Hong Kong students (Wheeler, Reis, \& Bond, 1989). This facility in stranger interactions has been described as a hallmark of individualism and may be bolstered by greater willingness to trust others - including strangers-among Americans as compared with Japanese (Yamagishi, 1988b).

Three studies used experimental manipulations to examine whether the influence of IND-COL on relationality is bound to social context. Findings suggest that IND and COL are rooted both in contexts and within individuals. In one study, both Chinese and American students high in COL favored their in-group especially when the group needed them (Y. Chen, Brockner, \& Katz, 1998). In a second study with multiethnic U.S. business students, McCusker and Bottom (2000, Study 1) found that allocentric individuals, that is, those who endorse more collective values, were generally more cooperative in a social dilemma task. On the other hand, idiocentric individuals, who endorse more individualistic values, cooperated only when instructions emphasized the team. A third study comparing American and Japanese participants found that high-performing Japanese left groups whenever possible, even when exiting was costly (Yamagishi, 1988a). Conversely, highperforming Americans exited only when the cost of leaving was low. In other words, both Americans and Japanese were willing to abandon a poorly performing group, but this tendency was even more pervasive among Japanese. Although this finding is contrary to the expected higher level of COL among Japanese, it is consistent with our meta-analytic results, which show that Americans are slightly higher in COL than are Japanese, especially when reliable scales are used. In the same vein, Yamagishi (1988a) highlighted the different circumstances under which Japanese and Americans left their groups. He suggested that in the real world, Japanese cooperation is not due to internalized collectivist values but instead is the result of structural monitoring and sanctioning of noncontributing free riders, which render leaving a poorly performing group or failing to contribute to the group unrealistic options.

Each of these studies provides a different perspective on the origins of collectivist behavior. Whereas the Y. Chen et al. (1998) results are compatible with the notion that $\mathrm{COL}$ is an internalized value system, Yamagishi (1988a) attributed cooperation to social structure and McCusker and Bottom (2000) highlighted the situated nature of cooperation. This trio of studies brings into sharp focus the difficulty of integrating the findings of studies that differ in level of analysis. Both Y. Chen et al. and Yamagishi used cross-national samples, but neither found that country had a main effect on cooperativeness. Only COL, when measured directly, made a difference. It is difficult to argue that COL is not an internalized value system when COL rather than country predicted results.

\section{Communication Styles}

Ten studies examined preferences for direct versus indirect communication. Four studies (three multinational and one Hawai- ian) assessed IND-COL and linked IND to direct and COL to indirect communication style. Indirect communication correlated negatively with IND and positively with COL among U.S., Australian, Japanese, and Korean students (Gudykunst et al., 1996). Similarly, participants with independent self-construals shifted toward direct requests when hints did not work, whereas those with interdependent self-construals continued to use indirect approaches. Among Hong Kong Chinese, Hawaiian, and mainland U.S. participants, those higher in interdependence preferred indirect communication even when using indirect communication did not succeed (M. Kim, Shin, \& Cai, 1998). IND correlated positively with a focus on clear, goal-oriented communication, whereas COL correlated positively with concern about a target's feelings and desires both to avoid negative evaluation and to minimize imposition on the target, among Korean, Japanese, Hawaiian, and mainland U.S. students (M. Kim et al., 1996). Finally, using a Hawaiian sample faced with a communicative bind (e.g., having to communicate negative news to a friend), M. Kim, Sharkey, and Singelis (1994) found that IND correlated with concern with message clarity, whereas COL predicted concern for the other person's feelings as well as concern with one's own self-presentation.

Six additional studies used country as a stand-in for IND, with similar results. In self-report studies, Americans were less obliging and used less avoiding, integrating, and compromising communication styles than Taiwanese students (Trubinsky, Ting-Toomey, \& Lin, 1991) and made fewer indirect influence attempts than Japanese students (Cocroft \& Ting-Toomey, 1994). Americans preferred direct contact or no contact with an opponent in a prisoner's dilemma paradigm, whereas Turkish students preferred an intermediary (Kozan \& Ergin, 1998). Americans praised group leaders who allowed group members to insult one another for providing an open forum, whereas Hong Kong Chinese students found such group leaders to be incompetent (M. H. Bond, Wan, Leung, \& Giacalone, 1985). Asked to bring to mind a recent conflict, American undergraduates were more likely to say that their goal was to attain justice; in contrast, Japanese undergraduates said they focused more on relationship goals (Ohbuchi, Fukushima, \& Tedeschi, 1999). Finally, American participants rated fast-talking male targets as more competent than targets speaking at a slower rate, but the reverse was true for Korean participants (Lee \& Boster, 1992). Presumably, rapid speech rate suggests to Americans that the speaker makes true and uncensored statements, whereas for Koreans, slow speech implies careful consideration of others and the context.

\section{Conflict Resolution Styles}

Sixteen studies explored the relation between IND-COL and collaboration-conflict style, including the nature of allocation norms. Seven studies assessed IND-COL directly and found evidence that IND-COL influences collaboration and conflict resolution style, although this effect may be specific to the interaction partner. Two reward allocation studies with Chinese, Japanese,

\footnotetext{
${ }^{8}$ The Pearson and Stephan (1998) negotiation preference study showed that high-COL groups, compared with high-IND groups, distinguished more between in-group and out-group partners.
} 
Korean, and U.S. undergraduates showed a positive correlation between COL and preference for equality rather than equity norms (Hui et al., 1991; Leung \& Iwawaki, 1988). Mexican and New Mexican undergraduates high in COL preferred accommodation as a mode of handling conflicts with family, friends, and coworkers (Gabrielidis, Stephan, Ybarra, Pearson, \& Villareal, 1997) and high-IND European American and Japanese undergraduates (assessed as independent self-construals) preferred confronting others and taking a turn rather than waiting for a turn in group interaction tasks (Oetzel, 1998a, 1998b). However, when conflict occurred with a fellow student, Canadians preferred negotiation and Nigerians preferred threats (Gire \& Carment, 1993); when conflict occurred with a neighbor, Canadians preferred arbitration and Nigerians preferred negotiation (Gire, 1997).

Seven studies used country as a stand-in for IND-COL, with five of these studies supporting the notion that Americans resolve conflicts and communicate in an individualistic style. In tit-for-tat and delayed tit-for-tat resource and public goods paradigms, recent immigrant adults from South Vietnam cooperated more than American undergraduates did (Parks \& Vu, 1994; Studies 1 and 2). In a scenario-based study, whereas all students rewarded targets who helped the group, American students were more sensitive to improved output and rewarded incrementally improved performance and Chinese students were less sensitive to this change in performance and did not provide rewards (M. H. Bond, Leung, \& Wan, 1982). Indian students of various ages were more likely to prefer an interpersonal strategy of conflict resolution compared with American students (Miller \& Bersoff, 1992). Finally, in two studies, European Americans were less likely to use equality norms in interactions with in-group members than were Chinese students (Leung \& Bond, 1982, 1984 Study 2). However, Wu (1995) did not find reliable differences between Taiwanese and Americans in a computerized resource allocation game.

Two studies examined the relation between cultural frame and use of equality-based norms in resolving discrimination issues. Ozawa, Crosby, and Crosby (1996) found that Japanese were more likely than Americans to support an equality-based solution to gender discrimination. Although this study did not assess IND or $\mathrm{COL}$, from the meta-analytic finding that Japanese are lower in IND but not lower in COL than Americans are, we infer that this difference is due to differences in IND. In fact, using the Ozawa et al. (1996) paradigm with Americans primed with IND or COL, Kemmelmeier (in press, Study 1) showed that Americans were more likely to endorse a merit-based solution when primed with IND and were more likely to endorse an equality-based solution when primed with COL.

\section{Organizational Research}

Organizational research is somewhat isolated within cultural and cross-cultural psychology both because scientists in nonorganizational domains rarely cite this research and because organizational researchers do not routinely cite nonorganizational cultural research. Even though Hofstede's (1980) analyses were organizational in nature, nonorganizational researchers typically do not focus on this aspect of his work. Generally, organizational researchers draw on a somewhat different theoretical base than other cultural or cross-cultural research. Thus, cultural organizational studies are often based in the work of Parsons and Shills (1951), who differentiate between individualists, motivated by their selfinterests and attainment of personal goals, and collectivists, motivated to promote the social system and collective interests. Moreover, organizational researchers define COL in terms of work groups rather than other kinds of groups. This means that COL scales used by organizational researchers differ from those used in other psychological research, with organizational researchers assessing IND and COL, for example, in terms of preferences for working alone or collaboratively in the workplace (Earley \& Gibson, 1998).

It is not entirely clear how COL defined within organizational research (preference for working in groups) relates to COL as defined in other areas of psychology (primarily in terms of sense of in-group duty or obligation, as described earlier). This is an important problem because in other areas of psychology, in-group duty and obligation often focus on groups other than workgroups (e.g., ethnic group, family, cultural group). Recall that in our meta-analyses we could not tease apart the effect of preference for working in groups from other components of COL for Korea, PR China, and Poland because all of the studies included preference for working in groups as part of the assessment of COL. For other comparisons results were mixed (see Tables 7 and 14). Therefore, organizational research linking workplace preferences to other aspects of COL is particularly valuable. Another advantage of this research base is that organizational researchers do not rely on undergraduate samples because the majority of organizational research focuses on adults in the workplace or on management students (8 of 16 studies).

\section{Working in Groups and Job Satisfaction}

We found eight studies relevant to this topic. Four studies that assessed IND-COL highlighted the influence of COL in the workplace. Higher workplace COL correlated with better job-related good citizenship among Americans (Moorman \& Blakely, 1995). Low COL predicted both a preference to work alone and better performance when working alone among American, Chinese, and Israeli managers (Earley, 1993). Low COL was associated with low levels of cooperation in large but not small groups (Wagner, 1995) and lower quality relationships within European American work groups (Eby \& Dobbins, 1997).

However, research in this domain does not provide evidence for a simple main effect relation between IND or COL and job performance or satisfaction but rather an interaction between social context and the effects of IND-COL. For U.S. managers, performance improved when instructions were focused on individual efficacy, congruent with a presumed cultural focus on IND. Conversely, for Chinese managers, performance improved when instructions were group focused, congruent with a presumed cultural focus on COL (Earley, 1994). A similar effect was shown in a within-U.S. business student simulation by Chatman and Barsade (1995) in which collectivists were more cooperative in a context that facilitated cooperative norms; no effect was shown for individualists.

Similarly, whereas COL may make the workplace more satisfying when work-related social networks are valued, IND may make the workplace more satisfying when the work itself becomes self-defining. Thus, a large-scale correlational study using data from 11.5 million employees from 650 companies in 45 countries 
found Hofstede's (1980) IND ratings to be marginally negatively correlated with work relationship satisfaction and communication (Hui, Yee, \& Eastman, 1995), favoring the proposition that IND does not enhance job satisfaction. Conversely, Hofstede (1980) found that people in individualistic countries put a greater emphasis on feelings of enjoyment at their workplace, favoring the proposition that IND elicits a focus on work as satisfying.

\section{Conflict Management and Negotiation Styles}

Eight studies sought to relate negotiation style and conflict management to IND-COL. Five of these studies assessed INDCOL directly and six studies used simulation tasks rather than paper-and-pencil attitude scales. First, in a negotiation simulation with a cross-national sample of managers, U.S. negotiators were more likely to endorse a self-interest negotiation schema than Japanese negotiators; IND partially mediated this relation between country and preferred style (Brett \& Okumura, 1998). However, another simulation examining performance among managers from the United States and Israel found that whether managers set their own standards did not systematically shift performance for those higher versus lower in COL (Erez \& Earley, 1987).

Cross-national simulations with undergraduates suggested differences in American negotiation style, related in part to cultural frame. First, emphasizing productivity increased use of equitybased rewards among Americans as well as Chinese, but placing an emphasis on fairness led to more equity-based rewards only among Americans. (C. C. Chen et al., 1998). This study also showed that only in Hong Kong were greater levels of COL associated with a preference for equality- rather than equity-based reward allocation. In a second simulation with American business undergraduates with work experience, COL correlated weakly with a preference for human resource management practices promoting job security, equal distribution of rewards, and formal evaluation practices that avoid direct confrontation (Ramamoorthy $\&$ Carroll, 1998). IND and COL also related to negotiation style in a sample of European American and Asian American undergraduates. Dyads high in COL negotiated a better deal for their group if the negotiating member expected to explain the negotiation process to the nonpresent member; dyads high in IND negotiated a better deal for their group when the negotiating partner did not expect to explain the negotiation process to the nonpresent partner (Gelfand \& Realo, 1999).

Only three studies did not assess IND-COL, and findings from these studies were less clear. P. B. Smith, Dugan, Peterson, and Leung (1998) relied on Hofstede's (1980) ratings of country-level IND to study how business managers in 23 countries dealt with conflict. The authors found that managers from low-IND countries preferred using formal rules and those from high-IND countries preferred basing the approach to conflict on personal experience. In a cross-national sample of bank employees, Lee and Rogan (1991) found that Koreans preferred to use compromise to handle conflict, whereas Americans preferred either to avoid the conflict or to confront the conflict directly. Finally, in a negotiation simulation Greek undergraduates were better at figuring out U.S. undergraduates' preferences than vice versa (Gelfand \& Christakopoulou, 1999).

\section{Summary}

Americans do feel obligated to family, often as strongly as others do, though they view these obligations as voluntary. However, it is unclear to what extent IND promotes intimacy and romantic love. The empirical base in this domain provides more support for COL-focused differences in relationality: Empirical literature on interactions with others found that Americans interacted with more groups, felt they could choose their groups more freely, and were more at ease with strangers than others. When assessed, COL related to favoring the in-group, preferring equality in in-group relationships, and accommodating in-group members. Assessed IND correlated with a preference for direct communication and "taking the floor" rather than waiting one's turn. Finally, organizational research showed that low COL correlated with a preference to work alone and performing better in solo tasks. Similarly, in within-U.S. studies, COL correlated positively with management practices that promote job security and equal distribution of rewards.

\section{DISCUSSION}

In this article we set out to (a) review the literature on individualism and collectivism and synthesize results from cross-cultural and within-U.S. ethnic research; (b) outline the degree of empirical support for theoretically derived implications of individualism and collectivism for self-concept, well-being, attribution style, and relationality; and (c) provide a comprehensive integration of the accumulated body of IND-COL research. To the extent that our review shows large and stable cross-cultural differences in INDCOL, and especially if these differences relate to differences in basic psychological domains, cultural psychology provides evidence for the need for multiple psychologies rather than a single, general psychology. Large and stable differences would imply that what has been taken to be a universal model of human psychology may simply be an American model, an individualistic vision of human nature. As detailed in the present article and reviewed below, we found support for reliable cultural differences. However, these differences were neither as large nor as systematic as often perceived.

In addition, the present review also showed that previous conclusions about cultural differences seemed to be based mainly on student samples, which presents a threat to the generalizability of the findings (cf. Sears, 1986). This reliance on college students is especially problematic for cultural research because studying college students may not provide an accurate representation of differences between societies. Specifically, it is plausible that college students' cultural values differ from the remainder of the population because, for example, they have a higher level of education and come from a higher socioeconomic background (Freeman, 1997; Hofstede, 1980; Triandis et al., 1990). These factors are likely to attenuate observed cultural differences. Although we did not find any evidence showing that cultural differences between students are smaller than cultural differences between adults, this issue remains one of the central challenges of an empirical cultural psychology.

\section{Implications of Meta-Analyses}

Despite a diverse tool kit of measures, there is a startling amount of cross-national convergence suggesting that IND and COL can 
be assessed at an individual level, with Americans emerging as higher in IND and lower in COL than others in a graphic display of the available data. This display also suggests that Americans are not higher in IND or lower in COL than most Europeans or those from English-speaking countries and are not different from Latin Americans in IND. It is premature, however, to conclude that Americans are no different from these groups in IND, because the stability of effects could be determined for only five countries from these regions: Australia, Germany, Mexico, Poland, and Puerto Rico. Overall, effect sizes for IND were smaller than were those for COL.

Effects within the United States were complex. Except for comparisons with Asian Americans, the meta-analyses did not support the notion of European Americans as a gold standard of individualism. Rather, African Americans emerged as the most individualistic U.S. group, with no significant differences between Latino Americans and European Americans. With regard to COL, European Americans were lower than Asian Americans and Latinos, but no different from African Americans. Because only comparisons between European Americans and Asian Americans showed the expected higher European American IND and lower COL, our findings call into question the practice of assuming U.S. minority groups are collectivists (see also Coon \& Kemmelmeier, 2001).

However, there are a number of problems with the current IND-COL database. First, the evidence for cross-national differences in IND and COL is seriously limited because of the heavy focus on four East Asian countries: Japan, Korea, Hong Kong, and PR China. Although research exists for many other countries, it is not in sufficient quantity to allow for the more in-depth analyses we conducted for the East Asian countries. Thus, for example, although evidence from three African countries supports the notion of high African COL, there are multiple studies only from Nigeria. It is unclear whether there are stable differences between Africa as a region overall and America. Similarly, for research on Latin and South America there are multiple studies only from Mexico and Puerto Rico, limiting our ability to make reliable generalizations about differences and similarities between people in the United States and people from Latin and South American countries.

Second, this paucity of research also means our analyses cannot shed any light on the question of whether ethnic minorities within the United States resemble the cultural groups outside of the United States from which they originated. Thus, it is unclear whether African Americans resemble Africans in their cultural orientation, as is sometimes proposed (e.g., Jones, 1986), or whether the African American cultural frame is distinctly American (or, given their higher individualism, quintessentially American). In addition, although a visual inspection of the cross-national data suggests that Latino Americans resemble people from Latin and South America (higher in COL but not necessarily lower in IND than European Americans), we again have too few data to support strong conclusions about the similarity of these groups.

Third, even within East Asia, the most studied region within IND-COL research, effect sizes are not uniform across countries. Overall, differences between Americans and Chinese (from PR China, Taiwan, or Hong Kong) are larger than differences between Americans and Japanese or Koreans. Effects for the other Asian countries with at least two data points represented (India, Indonesia, and Singapore) are mixed, with small effects for Indonesia and
Singapore, and effects for India falling between the large effects for Chinese peoples and the small effects found for Japan and Korea. Within Asia, COL may be more typical for Chinese, rather than Japanese or Korean cultures. Japanese and Korean cultures have common roots distinct from Chinese culture, and this finding points to the necessity of understanding differences between various Asian countries (see Dien, 1999) and resisting the implicit tendency found among American cultural psychologists to assume that all East Asians are the same.

Cognizant of these limitations, we turned to the potential moderating effects of scale reliability and scale content on the general findings. Given lack of sufficient studies from other countries, these analyses relied on the four commonly studied East Asian countries. With regard to scale reliability, COL moderator analyses showed that when reliable scales are used, effects become large for U.S.-PR China comparisons, become moderate for the Hong Kong comparisons, and shift from nonsignificant to significant but small for Japan and Korea. That is, even when reliable scales are used, Americans do not differ much in IND from Japanese and Koreans, for COL effects remain small for Koreans and actually flip for the Japanese-American comparisons, with Americans reporting slightly higher COL than Japanese.

Our review also suggests cautious interpretation of studies that presuppose IND-COL difference between Americans and Asians but did not directly assess IND or COL. U.S. (or Canada)-Japan comparisons without direct assessment (or priming) of IND-COL dominate cross-national self-concept research. Therefore, it is unclear whether IND and COL produced the cross-national differences found in self-concept between Japan and Canada and/or the United States. If the effects are not due to IND (or COL) but rather some country-specific differences between current Japanese and current Canadian societies, it becomes less clear how to generalize or apply these findings to other countries or situations.

Given that scale reliability tempered and qualified the general assumption of higher American IND and lower American COL, with stronger findings generally found with more reliable scales, it is particularly troubling that about half of the available crossnational research is based on measures with Cronbach reliabilities lower than .70, a conventional cutoff for acceptable reliability. For within-U.S. comparisons, two thirds of IND scales and over half of COL scales have low reliability. Although the current data cannot fully address the controversy about whether researchers should assess IND and COL as multifaceted constructs encompassing a broad range of values, attitudes, and behaviors or as more specifically focused dimensions, the finding that distinctions between groups are sharpened with more reliable scales is relevant because broad scales necessarily have lower reliability. Thus, rather than attempting to measure IND and COL as a multidimensional construct with a single mean score, it may be preferable to assess each hypothesized element separately with a highly reliable scale. This approach has the advantages of better measurement and greater theoretical clarity, isolating the "active ingredients" in crosscultural differences. Several researchers have recently adopted this approach. Triandis and colleagues (Singelis et al., 1995; Triandis \& Gelfand, 1998) proposed measuring IND and COL using four distinct subscales. Others have created scales that tap a single specific dimension of IND and COL using four distinct subscales. Others have created scales that tap a single specific dimension of 
IND and COL, such as relational interdependence (Cross, Bacon, \& Morris, 2000) and familialism (Lay et al., 1998).

Finally, we examined the moderating effect of scale content on the size of IND and COL differences. Our analyses revealed that IND scales almost universally share a common operationalization of IND as valuing personal independence, whereas COL scales share a common operationalization of COL as duty to in-group. Researchers often add other plausibly related content to this common core. Examples of this additional content for IND include valuing self-knowledge, direct communication, and personal privacy, and examples for COL include defining the self in context, seeking others' advice, and feeling related to one's groups. Although plausibly related to IND and COL, these additional factors, our analyses suggest, are likely to vary cross-culturally in the nature and extent of their relation to IND and COL.

Given this large heterogeneity in construct definition and scale content, it is unclear to what extent differences between groups or countries found with such idiosyncratic IND-COL scales do (or do not) relate to the individualism and collectivism of respondents. Idiosyncratic scale content may itself account for the heterogeneity sometimes found in the comparisons of various groups. For example, when researchers assess IND with items focused on valuing privacy, the difference between Americans and Japanese increases, but the difference between European Americans and Asian Americans decreases (and becomes nonsignificant).

Careful inspection of extant IND-COL scales suggests that researchers commonly operationalize IND and COL by tapping into a variety of plausible consequences of independence (e.g., preference for personal privacy) and duty to in-group (e.g., seeking others' advice). This approach is problematic because it gives rise to confusion between underlying cultural values, which are assumed to shape behaviors, and the behaviors themselves. The result is a potential tautology as IND and COL are first operationalized in terms of their consequences and are then correlated with these same consequences. We see this risk of circular reasoning as further support for our advocacy of narrowly defining IND and COL in terms of their core elements.

On the basis of our integration of the available data, we propose that the most basic way of defining and assessing IND is the extent to which personal uniqueness and independence is valued. Likewise, the most basic way of defining and assessing COL is the extent to which duty to in-group (and in cross-national comparisons, group harmony) is valued. Defining IND and COL in this way should not only result in measures that are more reliable but also provide a common metric with which to compare all societies. Further, this approach allows researchers to study whether IND and COL do in fact have the kinds of psychological consequences that they are currently assumed to have. This approach also clarifies that other forms of relationality should also be studied for their cultural variation-some are likely to relate to individualism and others to collectivism.

\section{Implications of Empirical Research}

Although some overlap exists, studies examining implications of IND and COL for basic psychological functioning form a largely independent body of research from studies simply assessing IND and COL differences between groups. The breadth of research on basic psychological implications of IND-COL, the lack of replication studies, and the diversity of dependent measures is striking and defies simple synthesis. Psychological content domain is confounded with sample type, level of analysis, and research methods. Specific single-country comparisons dominate certain domains of research (e.g., self-esteem research mostly compares the United States and Canada with Japan), and research comparing American subgroups is dispersed across a broad array of domains, with little replication. Moreover, assessment and manipulation of IND-COL is generally restricted to this more limited body of within-U.S. research.

Within research on the self, about three fourths of self-concept research is cross-national, and within-U.S. research focuses on personality traits. Thus, we do not know much about whether personality correlates of COL, such as need for affiliation, are generalizable outside the United States or are independent of the particular measures of IND and COL used. Self-concept and self-esteem researchers rarely assess IND or COL. In the area of self-concept research, the most common dependent variable was an open-ended self-concept description, although researchers also used self-esteem and various self-rated personality trait measures. Correlational designs and idiosyncratic coding schemes dominate self-concept and personality trait research. Finally, although research on self-esteem is predominantly experimental, this more rigorous method cannot overcome the limitations of lack of direct assessment of IND and COL and reliance on comparisons with only one country, namely, Japan.

In spite of this diversity, some coherence emerges. First, COL is associated with interpersonally focused personality traits such as warmth. The association between COL and describing the self with social rather than personal identities is much weaker, especially because researchers in this area use country as a stand-in for cultural difference. Similarly, self-esteem researchers use country as a marker for IND-COL. Even though their experimental evidence suggests Americans are more concerned about maintaining positive self-esteem than are Japanese, interpretation of national differences is unclear. Our meta-analyses found a small difference in IND between Japanese and Americans and no overall difference between Japanese and Americans except in research that used more reliable scales-in which case, Americans are higher in COL.

Like self-concept research, the majority of well-being research is correlational. However, research in this domain benefits from a large body of multinational research, with only about a third of the research focused on within-United States comparisons. With regard to the question of whether IND-COL influences well-being, recent research corroborates Hofstede's (1980) intuition that IND relates to social structural factors relevant to the workplace. This finding is important, in that it provides an important broadening of Hofstede's initial research, as well as surprising because these findings are based on a correlation between current socialstructural factors and societal-IND scores obtained almost 20 years ago. This result suggests more stability in Hofstede's initial IND scores than he himself believed would be the case. Further, a relation between Hofstede's IND score and average country-level life satisfaction ratings suggests that IND may increase well-being.

However, once a full set of society-level indicators (e.g., GNP, human rights observance) is controlled, Hofstede's (1980) IND score no longer relates to life satisfaction. It is not clear whether the absence of a relation is due to the use of this outdated indicator 
of IND or truly reflects that IND does not account for additional variance once researchers consider these other factors. What would constitute an appropriate test of the main effect of IND on national well-being is unclear because IND is likely both to be influenced by and to influence these society-level factors. In spite of this difficulty, within a given society, failure to attain culturally valued goals does seem to dampen well-being. Thus, putting aside the question of whether IND or COL has a main effect influence on well-being, researchers may be able to more fruitfully examine the influence of IND and COL on the processes by which well-being is constructed and maintained.

Third, with regard to attribution research, almost all of the research is experimental, but fewer than half of the studies assess IND-COL, instead using country as a stand-in for IND or COL. Studies that do assess IND-COL are primarily conducted within the United States, and within-U.S. studies make up about a third of the research base overall. However, this research does substantiate the proposed effects of IND-COL on attribution. Higher COL is associated with greater readiness to use contextual information. Conversely, higher IND Americans are more willing to process information based on traits, whether making attributions about people, information, or social obligation. With regard to social obligation specifically, those higher in COL do seem more responsive to others' needs across a diverse array of contextual cues.

Family and intimate relationships, intergroup relations, and organizational research formed the final psychological domain reviewed for evidence of IND-COL influences. Although differing in samples, research method, and design, research in this area dominates the field in terms of the sheer number of studies, as well as the number of conceptual replications and extensions of similar research. Because this domain is central to the nature of the IND-COL difference, we posited stark differences between collective and individualist focus. In fact, we did find evidence of a distinct COL relational style. IND makes salient the need to calibrate responses to individuals, regardless of their group membership, but COL highlights treating all in-group members equally while distinguishing between in- and out-group members. High IND is associated with direct communication, but high COL is associated with more indirect and face-saving communication style. Last, whereas IND is associated with equity norms, COL is associated with equality norms for in-group members.

Research on the workplace also highlights the complexity of the interface between societal values and individual perception. It is not that either IND or COL is more motivating or results in better performance. Rather, the impact of each orientation varies according to its prominence in the larger culture that contains each orientation. When the work environment matches an implicit larger societal focus on IND, for example, by emphasizing individual efficacy, an IND work focus may be more successful than a COL focus in motivating workers. Similarly, if the work environment matches an implicit larger societal focus on COL, by emphasizing cooperation, a COL work style may be more successful.

Perhaps the most surprising finding from this body of research is that relationality and family orientation did not emerge as closely linked to COL. Americans see themselves as choosing to be close to their family but not obligated to them. Similarly, Americans reported feeling close to members of their groups, enjoying being with them, and seeking other's advice. In that sense, Americans are relational but not necessarily collectivists.

As with the meta-analyses, these findings provide a striking example of the need for a tighter operationalization of IND-COL so that potential consequences of IND and COL can be studied empirically. In particular, relationality must be assessed as a possible consequence of IND rather than as a measure of COL. Americans are attuned to others, yet they are not collectivists in terms of the core elements of this concept-they do not feel obligated or duty-bound to their groups. If other elements of relationality, such as feeling close to members of the group, are included in operationalizations of COL, Americans will appear high in COL, attenuating a potentially large difference between Americans and East Asians. Thus, we speculate that when researchers assess duty to in-groups or preserving in-group harmony on the one hand and feeling close to members of one's groups on the other hand, group differences will emerge. When both elements are included in the same measure, it is impossible to assess existing differences in a meaningful way.

\section{Conclusions}

As a general package, the empirical evidence does support the notion that (European) Americans differ in IND and COL from others and that IND and COL do influence basic psychological processes. However, the empirical basis for this conclusion is not as firm as might be desired or as casual reading of textbooks in psychology would have the reader believe. Replications are rare, and often domain of research, sample, method, and design go hand in hand. Therefore, it is hard to say whether cultural differences attributed to difference in IND or COL are generalizable across populations or regions or whether differences found are limited to the countries studied in our meta-analyses.

As a result, at this time, it is impossible to tell the extent to which different cultural research methods - what we have termed direct assessment (directly assessing individual IND-COL), applying Hofstede (comparison of individuals or groups assumed to differ in IND-COL on the basis of Hofstede's [1980] work), and priming IND-COL (experimental manipulations of the salience of IND-COL) - produce the same effects. If they do produce similar effects, it is unclear whether it is by the same process. Thus, the current evidence cannot shed light on the quality or nature of the distinction between country-level individualism-collectivism, individual idiocentrism-allocentrism, and situationally elicited independence-interdependence. The future impact of cultural psychology will depend, in part, on clarifying the implications of these differences in levels of analysis and method for basic psychological functioning.

Given the current evidence, the following outline begins to emerge. At the societal level, surprisingly little research has focused on the structures that maintain and support IND-COL differences. However, we speculate that IND-focused and COLfocused societies differ in their organization, with COL-focused societies making obligation to groups salient and punishing those who do not promote in-group harmony (see, e.g., Yamagishi, 1988a). In the same way, we speculate that IND-focused societies use IND-centered practices and symbols to make personal uniqueness salient (see, e.g., Kemmelmeier \& Winter, 2000) and to punish those who do not separate themselves from others (Oyser- 
man \& Markus, 1993). That said, it is clear that societies do not equally enforce these cultural mandates on all members and do not equally provide the resources to enact culturally appropriate ways of being.

At the individual level, we can expect great variability; individuals differ in the extent that IND and COL, however termed, are chronically accessible. Chronically allocentric (interdependent) individuals are likely to think about the self and others differently than chronically idiocentric (independent) individuals. We have only begun to study the basic assumption that differences in how the self is conceptualized-as separate versus as a component of groups to which one owes duty and sacrifice - carry with them different ways of making sense of the self, well-being, relationships, and attribution.

Finally, we view the emerging research using priming to situationally cue IND and COL as a promising research strategy. The priming approach assumes that all people can think about the world in an IND or COL frame but differ in what is likely to come to mind. Because, as shown repeatedly in social cognition research, people base their judgments on what comes to mind at the time a judgment or decision is made, chronic differences in INDversus COL-based behavior ensue from differences in habitual focus on IND or COL. Thus, a priming perspective suggests that salient cultural perspectives influence behavior. We find this assumption plausible, yet we see a need for further research to identify what comes to mind when people from diverse cultures are primed to think in an individualistic or collectivistic manner. We are particularly concerned with the ambiguous nature of current priming techniques. Most of the manipulations used in these studies make salient relational aspects of the self (Gardner et al., 1999) and the way the self is similar to others (Trafimow et al., 1991), not obligation or duty to the in-group. This poses the question, To what extent do IND and COL cued situationally present an experimental analogue for cross-cultural differences? To the extent that relationality is not equivalent to collectivism, priming relationality may not be analogous to cross-cultural differences in collectivism. It is quite possible that that relationality, as primed in the experiment, is not equivalent to COL as measured in direct assessment techniques. Better understanding of the interface between methods is critical to the field of cultural psychology. Some emerging research suggests that priming does hold promise of identifying important process differences. For example, in our own lab, we have shown that when primed with independence versus interdependence, participants differ in their memory and information processing style. Specifically, interdependence priming increases sensitivity to contextual information, and these differences parallel those found when contrasting responses of Chinese and German participants - suggesting that priming and crosscultural processes are analogous (Haberstroh, Oyserman, Schwarz, \& Kühnen, in press).

Our main criticisms of the extant IND-COL literature are the overly broad and diffuse ways researchers define and assess these constructs and their apparent willingness to accept any crossnational difference as evidence of IND-COL processes. Low levels of consensus in definitions of IND-COL result in idiosyncratic operationalizations and assessments of these constructs. This broad conceptualization contributes to an emerging cultural tower of Babel in which cultural psychologists are quick to declare any cross-national difference to be "cultural" and any cultural differ- ence to be within the purview of IND-COL theory. This wide latitude is especially evident when researchers do not directly assess IND and COL and use cross-national or cross-group differences to infer that these differences are due to IND-COL-based differences in psychological processes. However, given this heterogeneity in implicit and explicit definitions of IND-COL, research methods, and level of analysis, emergence of some consistency in research findings in this field suggests that IND and COL are concepts worth saving.

In principle, the greatest strength of the IND-COL framework is its theoretical parsimony. From the myriad of possibilities, the model focuses on a few central dimensions of cultural difference that provide a powerful explanatory tool for understanding the variability in the behavior of individuals in different parts of the world. However, the value of this approach is contingent on the concepts of IND and COL being defined clearly, allowing them to be operationalized, assessed, and manipulated. Only under this condition will social scientists be able to evaluate the usefulness of the IND-COL framework. If IND and COL are not defined explicitly or if their definitions are overly inclusive, the scientific value of these constructs is seriously compromised.

Future research should refocus attention on the core elements of IND (independence and uniqueness) and COL (duty to in-group and, cross-nationally, maintaining harmony) if the IND-COL framework is to avoid becoming so content packed as to be theoretically empty. Other elements, such as the enjoyment of belonging to groups or seeking others' advice, have not been shown to be congruent with the core components. Our critique fits well with arguments by others, including Schwartz (1990), Triandis (1995), and Kagitcibasi (1994, 1997), who indicate problems with the broadness of definitions and lack of distinctions among levels of analysis. Yet, unlike Schwartz, our critique is not that IND and COL are simply one of many cultural differences. Diverging somewhat from Triandis, we do not conclude that IND and COL form inseparable and universal parts of greater cultural syndromes. Finally, whereas Kagitcibasi proposed that relationality is the core of COL, we propose that duty to in-group (and that cross-nationally, maintaining harmony) is core and that relationality should be studied separately (see also Kashima et al., 1995).

Thus, given this tentative state of the field, it seems premature to suggest a need for multiple psychologies for at least three reasons. First, observed effects tend not to be large; second, effects tend not to be replicated; and third, effects focus on either a particular country comparison or a particular aspect of functioning in the broader domain, leaving open questions of generalizability. As described previously, effect sizes are particularly small for IND. As a result, sweeping theoretical assumptions about the ways IND influences basic psychological functioning are built on a rather weak empirical foundation. In spite of some well-studied areas (e.g., self-esteem in Japan), significant findings often stand alone without a coherent body of sufficiently similar results. It is difficult to believe that findings using a particular measure in a particular country at a particular time constitute sufficient evidence of wide-ranging cultural differences in a domain. The press to explain specific findings within the rubric of IND-COL has resulted in noncritical acceptance of any difference whatsoever as corroborative evidence. In spite of these limitations, it is plausible that American and Western psychology are infused with an understanding of human nature based on individualism, raising the 
question of our ability to separate our current individualism-based way of understanding human nature from a yet to be developed collectivism-based approach. Psychologists reading this review should come away with renewed interest in understanding the social, interactive, and context-dependent nature of psychological functioning.

Even though it is not reasonable to propose the existence of multiple psychologies, our review clearly points out some ways in which IND and COL influence basic psychological domains. First, not everyone makes sense of the self in terms of high self-esteem or positive self-views, as Americans do (e.g., Heine, Lehman, Markus, \& Kitayama, 1999, suggest that Japanese do not). Second, far from being universally tied to IND or to COL, well-being is related to attaining culturally valued outcomes. In terms of attribution and cognitive style, not everyone spontaneously and persistently ignores contextual influence on human behavior as Americans do. Finally, people are likely to differ in what they understand to be reinforcing and rewarding and how they treat in-group as opposed to out-group members. Further, the body of cultural evidence does make clear the need to include relationality and desire for closeness to others as components of self-concept, well-being, and intergroup relations, whether considering these parts of a single psychology or as multiple psychologies. To answer the question with which we began, Americans are individualists as defined by their responses to IND scales, the way they define themselves, and what evidence they find convincing and motivating, but it is equally clear that Americans are relational and feel close to group members, seeking their advice.

\section{References}

References marked with an asterisk indicate articles included in the meta-analyses.

Agnew, C. R., \& Lee, B. (1997, May). Individualism in romantic relationships: Associations with commitment, satisfaction, and self-other inclusion. Paper presented at the annual meeting of the American Psychological Society, Washington, DC.

Al-Zahrani, S. S., \& Kaplowitz, S. A. (1993). Attributional biases in individualistic and collectivistic cultures: A comparison of Americans with Saudis. Social Psychology Quarterly, 56, 223-233.

*Arikawa, H., \& Templer, D. I. (1998). Comparison of Japanese and American college students on collectivism and social context of decision-making. Psychological Reports, 83, 577-578.

Arrindell, W. A., Hatzichristou, C., Wensink, J., Rosenberg, E., Van Twillert, B., Stedema, J., \& Meijer, D. (1997). Dimensions of national culture as predictors of cross-national differences in subjective wellbeing. Personality and Individual Differences, 23, 37-53.

Aron, A., Aron, E., \& Smollen, D. (1992). Inclusion of other in the self scale and the structure of interpersonal closeness. Journal of Personality and Social Psychology, 63, 596-612.

Bargh, J. A., Bond, R. N., Lombardi, W. J., \& Tota, M. E. (1986). The additive nature of chronic and temporary sources of construct accessibility. Journal of Personality and Social Psychology, 50, 869-878.

Baumeister, R. (1998). The self. In D. Gilbert, S. Fiske, \& G. Lindzey (Eds.), Handbook of social psychology (Vol. 1, pp. 680-740). New York: Oxford University Press.

Beck, A. T., Rush, A. J., Shaw, B. F., \& Emery, G. (1979). Cognitive therapy of depression. New York: Guilford Press.

Becker, B. J. (2000). Multivariate meta-analysis. In H. E. A. Tinsley \& S. D. Brown (Eds.), Handbook of applied multivariate statistics and mathematical modeling (pp. 499-525). San Diego, CA: Academic Press.
Befu, H. (1990). Conflict and non-Weberian bureaucracy in Japan. In S. N. Eisenstadt \& A. Ben-Ari (Eds.), Japanese models of conflict resolution (pp. 162-191). New York: Kegan Paul International.

Bellah, R., Madsen, R., Sullivan, W., Swidler, A., \& Tipton, S. (1985). Habits of the heart: Individualism and commitment in American life. Berkeley: University of California Press.

*Benet-Martinez, V. (2000). Examining the interplay between personality and culture: Personality correlates of cultural, ethnic, and linguistic orientations. Unpublished manuscript, University of Michigan, Ann Arbor.

Bersoff, D. M., \& Miller, J. G. (1993). Culture, context, and the development of moral accountability judgments. Developmental Psychology, 29, 664-676.

Berzonsky, M. D. (1992). Identity style inventory (rev.). Unpublished manuscript, State University of New York College at Cortland.

Bettencourt, B. A., \& Dorr, N. (1997). Collective self-esteem as a mediator of the relationship between allocentrism and subjective well-being. Personality and Social Psychology Bulletin, 23, 955-964.

*Bierbrauer, G., Heyer, H., \& Wolfradt, V. (1994). Measurement of normative and evaluative aspects of individualistic and collectivistic orientations: The Cultural Orientation Scale (COS). In U. Kim, H. C. Triandis, C. Kagitcibasi, S. Choi, \& G. Yoon (Eds.), Individualism and collectivism: Theory, method, and applications (pp. 189-199). Thousand Oaks, CA: Sage.

*Bloom, K., Oyserman, D., Menard, K., \& Masataka, N. (2000). Sociocultural influences on parenting possible selves among Canadian and Japanese women. Unpublished manuscript, University of Waterloo, Waterloo, Ontario, Canada.

Bond, M. H., \& Cheung, T. (1983). College students' spontaneous selfconcept: The effect of culture among respondents in Hong Kong, Japan, and the United States. Journal of Cross-Cultural Psychology, 14, 153171.

Bond, M. H., Leung, K., \& Wan, K. C. (1982). How does cultural collectivism operate? The impact of task and maintenance contributions on reward distributions. Journal of Cross-Cultural Psychology, 13, 186-200.

Bond, M. H., Wan, K., Leung, K., \& Giacalone, R. A. (1985). How are responses to verbal insult related to cultural collectivism and power distance? Journal of Cross-Cultural Psychology, 16, 111-127.

Bond, R., \& Smith, P. B. (1996). Culture and conformity: A meta-analysis of studies using Asch's (1952b, 1956) line judgment task. Psychological Bulletin, 119, 111-137.

Bontempo, R. (1993). Translation fidelity of psychological scales: An item response theory analysis of an individualism-collectivism scale. Journal of Cross-Cultural Psychology, 24, 149-166.

Bontempo, R., Lobel, S., \& Triandis, H. (1990). Compliance and value internalization in Brazil and the U.S.: Effects of allocentrism and anonymity. Journal of Cross-Cultural Psychology, 21, 200-213.

Bradburn, N. M. (1969). The structure of psychological well-being. Chicago: Aldine.

Braiker, H., \& Kelley, H. (1979). Conflict in the development of close relationships. In R. Burgess \& T. Huston (Eds.), Social exchange in developing relationships. New York: Academic Press.

Breckler, S. J., Greenwald, A. G., \& Wiggins, E. C. (1986). Public, private, and collective self-evaluation: Measurement of individual differences. Paper presented at the International Research and Exchange Board Conference on Self and Social Involvement, Princeton, NJ.

Breer, P. E., \& Locke, A. (1965). Task experience as a source of attitudes. Homewood, IL: Dorsey Press.

*Brett, J. M., \& Okumura, T. (1998). Inter- and intracultural negotiation: U.S. and Japanese negotiators. Academy of Management Journal, 41, 495-510.

Brewer, M. B., \& Gardner, W. (1996). Who is this "we"? Levels of 
collective identity and self-representations. Journal of Personality and Social Psychology, 71, 83-93.

Burke, E. (1973). Reflections on the revolution in France. Garden City, NY: Anchor Press. (Original work published 1790)

Campbell, A., Converse, P. E., \& Rodgers, W. L. (1976). The quality of American life. New York: Russell Sage.

*Carpenter, S., \& Radhakrishnan, P. (1998a, February). Cognitive representations of friends: Influence of culturelethnicity and individual differences. Paper presented at the annual meeting of the Society for Cross-Cultural Research, St. Petersburg, FL.

*Carpenter, S., \& Radhakrishnan, P. (1998b, May). Effects of interdependence on conceptualizations of self and groups: Cultural and individual level analyses. Paper presented at the annual meeting of the American Psychological Society, Washington, DC.

*Carpenter, S., \& Radhakrishnan, P. (1999, February). Need for belonging: Perceptions of family, friends, ethnicity, gender, age, and student groups. Paper presented at the annual meeting of the Society for CrossCultural Research, Santa Fe, NM.

*Carter, K., \& Dinnel, D. L. (1997, April). Self-esteem conceptualization: A comparison of American and Japanese values. Poster presented at the annual meeting of the Western Psychological Association, Seattle, WA.

CBS News. (1998). "60 Minutes" poll (ICPSR version) [Computer file]. Ann Arbor, MI: Inter-University Consortium for Political and Social Research.

Chan, D. K. (1994). COLINDEX: A refinement of three collectivism measures. In U. Kim, H. C. Triandis, C. Kagitcibasi, S. Choi, \& G. Yoon (Eds.), Individualism and collectivism: Theory, method, and applications (pp. 200-210). Thousand Oaks, CA: Sage.

Chatman, J. A., \& Barsade, S. G. (1995). Personality, organizational culture, and cooperation: Evidence from a business simulation. Administrative Science Quarterly, 40, 423-443.

Cheek, J. M., \& Tropp, L. R. (1995). The aspects of identity questionnaire: History and bibliography. Unpublished manuscript, Wellesley College, Wellesley, MA.

*Chen, Y., Brockner, J., \& Katz, T. (1998). Toward an explanation of cultural differences in in-group favoritism: The role of individual versus collective primacy. Journal of Personality and Social Psychology, 75, $1490-1502$.

*Chen, C. C., Meindl, J. R., \& Hui, H. (1998). Deciding on equity or parity: A test of situational, cultural, and individual factors. Journal of Organizational Behavior, 19, 115-129.

*Chew, K. H. (1996). Beyond individualism-collectivism: Additional constructs to consider. Unpublished doctoral dissertation, University of Oregon, Eugene.

Chiasson, N., Dube, L., \& Blondin, J. (1996). A look into the folk psychology of four cultural groups. Journal of Cross-Cultural Psychology, 27, 673-691.

Choi, I., \& Nisbett, R. E. (1998). Situational salience and cultural differences in the correspondence bias and the actor-observer bias. Personality and Social Psychology Bulletin, 24, 949-960.

Choi, I., Nisbett, R. E., \& Norenzayan, A. (1999). Causal attribution across cultures: Variation and universality. Psychological Bulletin, 125, 47-63.

*Cialdini, R. B., Wosinska, W., Barrett, D. W., Butner, J., \& GornikDurose, M. (1999). Compliance with a request in two cultures: The differential influence of social proof and commitment/consistency on collectivists and individualists. Personality and Social Psychology Bulletin, 25, 1242-1253.

*Cocroft, B. K., \& Ting-Toomey, S. (1994). Facework in Japan in the United States. International Journal of Intercultural Relations, 18, 469506.

Cohen, J. (1988). Statistical power analysis for the behavioral sciences (2nd ed.). Hillsdale, NJ: Erlbaum.

*Coon, H. M., \& Kemmelmeier, M. (2001). Cultural orientations in the
United States: (Re)-examining differences among ethnic groups. Journal of Cross-Cultural Psychology, 32, 348-364.

Coon, H. M., \& Oyserman, D. (2001). The measurement of individualism and collectivism. Manuscript in preparation.

Cousins, S. D. (1989). Culture and self-perception in Japan and the United States. Journal of Personality and Social Psychology, 56, 124-131.

Crandall, C. S., Preisler, J. J., \& Aussprung, J. (1992). Measuring life event stress in the lives of college students: The undergraduate stress questionnaire (USQ). Journal of Behavioral Medicine, 15, 627-662.

Cross, S. E., Bacon, P. L., \& Morris, M. L. (2000). The relationalinterdependent self-construal and relationships. Journal of Personality and Social Psychology, 78, 791-808.

Cross, S. E., \& Madson, L. (1997). Models of the self: Self-construals and gender. Psychological Bulletin, 122, 5-37.

Curry, R. O., \& Valois, K. E. (1991). The emergence of an individualist ethos in American society. In R. O. Curry \& L. B. Goodheart (Eds.), American chameleon: Individualism in trans-national context (pp. 2043). Kent, OH: Kent State University Press.

Dabul, A. J., Bernal, M. E., \& Knight, G. P. (1995). Allocentric and idiocentric self-description and academic achievement among Mexican American and Anglo American adolescents. Journal of Social Psychology, 135, 621-630.

de Tocqueville, A. (1969). Democracy in America. Garden City, NY: Anchor Press. (Original work published 1835)

Dhawan, N., Roseman, I. J., Naidu, R. K., Thapa, K., \& Rettek, S. I. (1995). Self-concepts across two cultures: India and the United States. Journal of Cross-Cultural Psychology, 26, 606-621.

Dien, D. S. (1999). Chinese authority-directed orientation and Japanese peer-group orientation: Questioning the notion of collectivism. Review of General Psychology, 3, 372-385.

Diener, E., \& Diener, M. (1995). Cross-cultural correlates of life satisfaction and self-esteem. Journal of Personality and Social Psychology, 68, 653-663.

Diener, E., Diener, M., \& Diener, C. (1995). Factors predicting the subjective well-being of nations. Journal of Personality and Social Psychology, 69, 851-864.

Diener, E., Emmons, R. A., Larsen, R. J., \& Griffin, S. (1985). The Satisfaction With Life Scale. Journal of Personality Assessment, 49, 71-75.

*Dinnel, D. L., \& Kleinknecht, R. A. (1999, April). A cross-cultural comparison of social anxiety symptoms. Paper presented at the annual meeting of the Western Psychological Association, Irvine, CA.

Dion, K. K., \& Dion, K. L. (1975). Self-esteem and romantic love. Journal of Personality, 43, 39-57.

Dion, K. K., \& Dion, K. L. (1991). Psychological individualism and romantic love. Journal of Social Behavior and Personality, 6, 17-33.

Doherty, R. W., Hatfield, E., Thompson, K., \& Choo, P. (1994). Cultural and ethnic influences on love and attachment. Personal Relationships, 1 , 391-398.

Duff, K. J., \& Newman, L. S. (1997). Individual differences in the spontaneous construal of behavior: Idiocentrism and the automatization of the trait inference process. Social Cognition, 15, 217-241.

Durkheim, E. (1933). The division of labor in society. New York: Macmillan. (Original work published 1887)

*Earley, P. C. (1989). Social loafing and collectivism: A comparison of the United States and the People's Republic of China. Administrative Science Quarterly, 34, 565-581.

*Earley, P. C. (1993). East meets West meets Mideast: Further explorations of collectivistic and individualistic work groups. Academy of Management Journal, 36, 319-348.

*Earley, P. C. (1994). Self or group? Cultural effects of training on self-efficacy and performance. Administrative Science Quarterly, 39, 89-117.

Earley, P. C., \& Gibson, C. B. (1998). Taking stock in our progress on 
individualism-collectivism: 100 years of solidarity and community. Journal of Management, 24, 265-304.

*Ebreo, A. (1998). Subjective culture, perceived social support, and adaptive coping: A multi-ethnic study of the transition to college. Unpublished doctoral dissertation, University of Illinois, Urbana-Champaign.

Eby, L. T., \& Dobbins, G. H. (1997). Collectivistic orientation in teams: An individual and group-level analysis. Journal of Organizational Behavior, 18, 275-295.

Elizur, D. (1979). Assessing achievement motive of American and Israeli managers: Design and application of a three-facet measure. Applied Psychological Measurement, 3, 201-212.

Endo, Y., Heine, S. J., \& Lehman, D. R. (2000). Culture and positive illusions in close relationships: How my relationships are better than yours. Personality and Social Psychology Bulletin, 26, 1571-1586.

Erez, M., \& Earley, P. C. (1987). Comparative analysis of goal-setting strategies across cultures. Journal of Applied Psychology, 72, 658-665.

Fijneman, Y. A., Willemsen, M. E., \& Poortinga, Y. H. (1996). Individualism-collectivism: An empirical study of a conceptual issue. Journal of Cross-Cultural Psychology, 27, 381-402.

Fiske, A. P. (1992). The four elementary forms of sociality: Framework for a unified theory of social relations. Psychological Review, 99, 689-723.

Fiske, A. P., Kitayama, S., Markus, H. R., \& Nisbett, R. E. (1998). The cultural matrix of social psychology. In D. Gilbert, S. Fiske, \& G. Lindzey (Eds.), Handbook of social psychology (4th ed., Vol. 2, pp. 915-981). Boston: McGraw-Hill.

*Freeberg, A. L., \& Stein, C. H. (1996). Felt obligations towards parents in Mexican-American and Anglo-American young adults. Journal of Social and Personal Relationships, 13, 457-471.

Freeman, M. A. (1997). Demographic correlates of individualism and collectivism. Journal of Cross-Cultural Psychology, 28, 321-341.

Gabrielidis, C., Stephan, W. G., Ybarra, O., Pearson, V. M. D., \& Villareal, L. (1997). Preferred styles of conflict resolution: Mexico and the United States. Journal of Cross-Cultural Psychology, 28, 661-677.

Gaertner, L., Sedikides, C., \& Graetz, K. (1999). In search of self-definition: Motivational primacy of the individual self, motivational primacy of the collective self, or contextual primacy? Journal of Personality and Social Psychology, 76, 5-18.

*Gaines, S. O., Gilstrap, S., Kim, M., Yi, J., Rusbalt, C. E., Holcomb, D., et al. (1999, June). Cultural value orientations: Measurement and manifestation in responses to accommodative dilemmas. Paper presented at the joint conference of the International Network on Personal Relationships and the International Society for the Study of Personal Relationships, University of Louisville, Louisville, KY.

*Gaines, S. O., Marelich, W. D., Bledsoe, K. L., Steers, W. N., Henderson, M. C., Granrose, C. S., et al. (1997). Links between race/ethnicity and cultural values as mediated by racial/ethnic identity and moderated by gender. Journal of Personality and Social Psychology, 72, 1460-1476.

*Gardner, W., Gabriel, S., \& Lee, A. (1999). "I" value freedom but "we" value relationships: Self-construal priming mirrors cultural differences in judgment. Psychological Science, 10, 321-326.

Gelfand, M. J., \& Christakopoulou, S. (1999). Culture and negotiator cognition: Judgment accuracy and negotiation processes in individualistic and collectivistic cultures. Organizational Behavior \& Human Decision Processes, 79, 248-269.

Gelfand, M. J., \& Realo, A. (1999). Individualism-collectivism and accountability in intergroup negotiations. Journal of Applied Psychology, 84, 721-736.

Gelfand, M. J., Spurlock, D., Sniezek, J. A., \& Shao, L. (2000). Culture and social prediction: The role of information in enhancing confidence in social predictions in the U.S. and China. Journal of Cross-Cultural Psychology, 31, 498-516.

*Gire, J. T. (1997). The varying effect of individualism-collectivism on preference for methods of conflict resolution. Canadian Journal of Behavioural Science, 29, 38-43.
*Gire, J. T., \& Carment, D. W. (1993). Dealing with disputes: The influence of individualism-collectivism. Journal of Social Psychology, $133,81-95$.

Glaser, R., \& Glaser, C. (1991). Negotiation style profile. King of Prussia, PA: Organization Design and Development.

Gleser, L. J., \& Olkin, I. (1994). Stochastically dependent effect sizes. In H. Cooper \& L. V. Hedges (Eds.), Handbook of research synthesis (pp. 339-355). New York: Russell Sage Foundation.

Gohm, C. L., Oishi, S., Darlington, J., \& Diener, E. (1998). Culture, parental conflict, parental marital status, and the subjective well-being of young adults. Journal of Marriage and the Family, 60, 319-334.

*Grimm, S. D., Church, A. T., Katigbak, M. S., \& Reyes, J. A. S. (1999). Self-described traits, values, and moods associated with individualism and collectivism. Journal of Cross-Cultural Psychology, 30, 466-500.

Gudykunst, W. B., Gao, G., Schmidt, K. L., Nishida, T., Bond, M. H., Leung, K., et al. (1992). The influence of individualism-collectivism, self-monitoring, and predicted-outcome value on communication in ingroup and outgroup relationships. Journal of Cross-Cultural Psychology, 23, 196-213.

*Gudykunst, W. B., Matsumoto, Y., Ting-Toomey, S., Nishida, T., Kim, K., \& Heyman, S. (1996). The influence of cultural individualismcollectivism, self-construals, and individual values on communication styles across cultures. Human Communication Research, 22, 510-543.

Gudykunst, W. B., Yoon, Y., \& Nishida, T. (1987). The influence of individualism-collectivism on perceptions of communication in ingroup and outgroup relationships. Communication Monographs, 54, 295-306.

Haberstroh, S., Oyserman, D., Schwarz, N., \& Kühnen, U. (in press). Is the interdependent self a better communicator than the independent self? Self-construal and the observation of conversational norms. Journal of Experimental Social Psychology.

Hamaguchi, E. (1977). Nihonrashisa no saihakken [A rediscovery of Japaneseness]. Tokyo: Nihon Keizai Shinbunsha.

Han, S., \& Shavitt, S. (1994). Persuasion and culture: Advertising appeals in individualistic and collectivistic countries. Journal of Experimental Social Psychology, 30, 326-350.

Hedges, L. V., \& Olkin, I. (1985). Statistical methods for meta-analysis. Orlando, FL: Academic Press.

Heine, S. J., \& Lehman, D. R. (1995). Cultural variation in unrealistic optimism: Does the West feel more vulnerable than the East? Journal of Personality and Social Psychology, 68, 595-607.

Heine, S. J., Lehman, D. R., Markus, H. R., \& Kitayama, S. (1999). Is there a universal need for positive self-regard? Psychological Review, 106, 766-794.

Heine, S. J., Kitayama, S., Lehman, D., Takata, T., \& Ide, E. (1998, August). Self-enhancement and self-improvement: Two ways to motivate the self. Paper presented at the Stanford Mini-Conference on Cultural Psychology, Palo Alto, CA.

Heine, S. J., Takata, T., \& Lehman, D. R. (2000). Beyond self-presentation: Evidence for self-criticism among Japanese. Personality and Social Psychology Bulletin, 26, 71-78.

*Hetts, J. J., Sakuma, M., \& Pelham, B. W. (1999). Two roads to positive regard: Implicit and explicit self-evaluation and culture. Journal of Experimental Social Psychology, 35, 512-559.

Hinkle, S., Taylor, L. A., Fox-Cardamone, D. L., \& Crook, K. F. (1989). Intragroup identification and intergroup differentiation: A multicomponent approach. British Journal of Social Psychology, 28, 305-317.

Hobart, C. W. (1958). The incidence of romanticism during courtship. Social Forces, 36, 362-367.

Hofstede, G. (1980). Culture's consequences. Beverly Hills, CA: Sage.

Hofstede, G. (1991). Cultures and organizations: Software of the mind. London, England: McGraw-Hill.

Hsu, F. L. K. (1983). Rugged individualism reconsidered. Knoxville: University of Tennessee Press.

Hui, C. H. (1984). Individualism-collectivism: Theory, measurement, and 
its relation to reward allocation. Unpublished doctoral dissertation, University of Illinois, Urbana-Champaign.

*Hui, C. H. (1988). Measurement of individualism-collectivism. Journal of Research in Personality, 22, 17-36.

*Hui, C. H., Triandis, H. C., \& Yee, C. (1991). Cultural differences in reward allocation: Is collectivism the explanation? British Journal of Social Psychology, 30, 145-157.

Hui, C. H., \& Villareal, M. J. (1989). Individualism-collectivism and psychological needs: Their relationships in two cultures. Journal of Cross-Cultural Psychology, 20, 310-323.

Hui, C. H., Yee, C., \& Eastman, K. L. (1995). The relationship between individualism-collectivism and job satisfaction. Applied Psychology: An International Review, 44, 276-282.

Hunter, J. E., Schmidt, F. L., \& Jackson, G. B. (1982). Meta-analysis: Cumulating research findings across studies. Beverly Hills, CA: Sage. Inglehart, R. (1997). Modernization and postmodernization. Princeton, NJ: Princeton University Press.

Ip, G. W. M., \& Bond, M. H. (1995). Culture, values, and the spontaneous self-concept. Asian Journal of Psychology, 1, 29-35.

Jackson, D. N. (1972). Personality Research Form manual. Goshen, NY: Research Psychologists Press.

Jackson, J. W., \& Smith, E. R. (1999). Conceptualizing social identity: A new framework and evidence for the impact of different dimensions. Personality and Social Psychology Bulletin, 25, 120-135.

Ji, L.-J., Schwarz, N., \& Nisbett, R. E. (2000). Culture, autobiographical memory, and behavioral frequency reports: Measurement issues in cross-cultural studies. Personality and Social Psychology Bulletin, 26, 585-593.

Johnson, B. T. (1993). DSTAT 1.10: Software for the meta-analytic review of research literatures. Hillsdale, NJ: Erlbaum.

Johnson, C. A., Southwick, S., Carlson, D., \& Hsai, C. (1997, August). Independent and interdependent self-construals and the self-references and group-reference effects. Paper presented at the 105th annual Conference of the American Psychological Association, Chicago, IL.

Jones, J. M. (1986). Racism: A cultural analysis of the problem. In J. Dovidio \& S. L. Gaertner (Eds.), Prejudice, discrimination, and racism (pp. 279-314). Orlando, FL: Academic Press.

Jourard, S. M., \& Lasakow, P. (1958). Some factors in self-disclosure. Journal of Abnormal and Social Psychology, 56, 91-98.

Kagitcibasi, C. (1987). Individual and group loyalties: Are they compatible? In C. Kagitcibasi (Ed.), Growth and progress in cross-cultural psychology. Lisse, the Netherlands: Swets \& Zeitlinger.

Kagitcibasi, C. (1994). A critical appraisal of individualism and collectivism: Toward a new formulation. In U. Kim, H. C. Triandis, C. Kagitcibasi, S.-C. Choi, \& G. Yoon (Eds.), Individualism and collectivism: Theory, method, and applications (pp. 52-65). Thousand Oaks, CA: Sage.

Kagitcibasi, C. (1997). Individualism and collectivism. In J. W. Berry, M. H. Segall, \& C. Kagitcibasi (Eds.), Handbook of cross-cultural psychology: Vol. 3. Social behavior and applications (pp. 1-49). Boston: Allyn \& Bacon.

*Kashima, Y., Yamaguchi, S., Kim, U., Choi, S., Gelfand, M. J., \& Yuki, M. (1995). Culture, gender and self: A perspective from individualismcollectivism research. Journal of Personality and Social Psychology, 69, 926-937.

Kato, K., \& Markus, H. (1993). The role of possible selves in memory. Psychologia, 36, 73-83.

Kemmelmeier, M. (in press). Individualism and attitudes toward affirmative action: Evidence from priming studies. Basic and Applied Social Psychology.

*Kemmelmeier, M., Burnstein, E., Kanagawa, C., Krumov, K., Genkova, P., Hirshberg, M., et al. (2001). Authoritarianism, collectivism, and individualism in seven nations. Manuscript submitted for publication.

Kemmelmeier, M., Burnstein, E., \& Peng, K. (1999). Individualism and authoritarianism shape attitudes toward physician-assisted suicide. Journal of Applied Social Psychology, 29, 2613-2631.

Kemmelmeier, M., Sanchez-Burks, J., Cytron, A., \& Coon, H. M. (1998, August). Individualism and romantic love: A comparison of two hypotheses. Poster presented at the 106th Annual Convention of the American Psychological Association, San Francisco, CA.

Kemmelmeier, M., Wieczorkowska, G., Erb, H.-P., \& Burnstein, E. (in press). Individualism, authoritarianism, and attitudes toward assisted death: Cross-cultural, cross-regional and experimental evidence. Journal of Applied Social Psychology.

Kemmelmeier, M., \& Winter, D. G. (2000). What's in an American flag? National symbols prime cultural self-construals. Unpublished manuscript, University of Michigan, Ann Arbor.

*Kim, M., Hunter, J. E., Miyahara, A., \& Horvath, A. (1996). Individualvs. culture-level dimensions of individualism and collectivism: Effects on preferred conversational styles. Communication Monographs, 63, $28-49$.

Kim, M., Sharkey, W. F., \& Singelis, T. M. (1994). The relationship between individuals' self-construals and perceived importance of interactive constraints. International Journal of Intercultural Relations, 18, $117-140$.

Kim, M., Shin, H., \& Cai, D. (1998). Cultural influences on the preferred forms of requesting and re-requesting. Communication Monographs, 65 , $47-66$.

Kim, U. (1994). Individualism and collectivism: Conceptual clarification and elaboration. In U. Kim, H. C. Triandis, C. Kagitcibasi, S. Choi, \& G. Yoon (Eds.), Individualism and collectivism: Theory, method, and applications (pp. 19-40). Thousand Oaks, CA: Sage.

Kitayama, S., Markus, H. R., Matsumoto, H., \& Norasakkunkit, V. (1997). Individual and collective process in the construction of the self: Selfenhancement in the United States and self-criticism in Japan. Journal of Personality and Social Psychology, 72, 1245-1267.

Kitayama, S., Markus, H. R., Tummala, P., Kurokawa, M., \& Kato, K. (1991). Self-other similarity judgments depend on culture. Unpublished manuscript, Kyoto University, Kyoto, Japan.

*Kleinknecht, R. A., Dinnel, D. A., Kleinknecht, E. E., Hiruma, N., \& Harada, N. (1997). Cultural factors in social anxiety: A comparison of social phobia symptoms and Taijin Kyofusho. Journal of Anxiety Disorders, 11, 157-177.

Kohn, P. M., Lafreniere, K., \& Gurevich, M. (1990). The inventory of college students' recent life experiences: A decontaminated hassles scale for a special population. Journal of Behavioral Medicine, 13, 619-631.

Kozan, M. K., \& Ergin, C. (1998). Preference for third party help in conflict management in the United States and Turkey: An experimental study. Journal of Cross-Cultural Psychology, 29, 525-539.

Knox, D. H., \& Sporakowski, M. J. (1968). Attitudes of college students towards love. Journal of Marriage and the Family, 30, 638-642.

*Krull, D. S., Loy, M. H., Lin, J., Wang, C., Chen, S., \& Zhao, X. (1999). The fundamental attribution error: Correspondence bias in individualist and collectivist cultures. Personality and Social Psychology Bulletin, 25, $1208-1219$.

Kuhn, M. H., \& McPartland, T. (1954). An empirical investigation of self-attitudes. American Sociological Review, 19, 68-76.

*Kwan, V. S. Y., Bond, M. H., \& Singelis, T. M. (1997). Pancultural explanations for life satisfaction: Adding relationship harmony to selfesteem. Journal of Personality and Social Psychology, 73, 1038-1051.

Kwan, V. S. Y., \& Singelis, T. M. (1998, August). Pancultural explanations for life satisfaction: Adding relationship harmony to self-esteem. Paper presented at the Stanford Mini-Conference on Cultural Psychology, Palo Alto, CA.

Lay, C., Fairlie, P., Jackson, S., Ricci, T., Eisenberg, J., Sato, T., et al. (1998). Domain-specific allocentrism-idiocentrism: A measure of family connectedness. Journal of Cross-Cultural Psychology, 29, 434-460. Lee, F., Hallahan, M., \& Herzog, T. (1996). Explaining real-life events: 
How culture and domain shape attributions. Personality and Social Psychology Bulletin, 22, 732-741.

Lee, H. O., \& Boster, F. J. (1992). Collectivism-individualism in perceptions of speech rate: A cross-cultural comparison. Journal of CrossCultural Psychology, 23, 377-388.

Lee, H. O., \& Rogan, R. G. (1991). A cross-cultural comparison of organizational conflict management behaviors. International Journal of Conflict Management, 2, 181-199.

Lee, Y.-T., Kleinbach, R., Hu, P. C., Peng, Z. Z., \& Chen, X. Y. (1996). Cross-cultural research on euthanasia and abortion. Journal of Social Issues, 52, 143-168.

Leung, K. (1989). Cross-cultural differences: Individual-level vs. culturallevel analysis. International Journal of Psychology, 24, 703-719.

Leung, K., \& Bond, M. H. (1982). How Chinese and Americans reward task-related contributions: A preliminary study. Psychologia, 25, 32-39.

Leung, K., \& Bond, M. H. (1984). The impact of cultural collectivism on reward allocation. Journal of Personality and Social Psychology, 47, 793-804.

*Leung, K., \& Iwawaki, S. (1988). Cultural collectivism and distributive behavior. Journal of Cross-Cultural Psychology, 19, 35-49.

Levine, R., Sato, S., Hashimoto, T., \& Verma, J. (1995). Love and marriage in eleven cultures. Journal of Cross-Cultural Psychology, 26, $554-571$.

*Liang, S. (1997). Self-disclosure and conflict management: A comparison of Chinese and American couples. Unpublished doctoral dissertation, Texas Tech University, Lubbock.

Lin, Y. W., \& Rusbult, C. E. (1995). Commitment to dating relationships and cross-sex friendships in America and China. Journal of Social and Personal Relationships, 12, 7-26.

Little, T. D., Oettingen, G., Stetsenko, A., \& Baltes, P. B. (1995). Children's action-control beliefs about school performance: How do American children compare with German and Russian children? Journal of Personality and Social Psychology, 69, 686-700.

Luhtanen, R., \& Crocker, J. (1992). A collective self-esteem scale: Selfevaluation of one's social identity. Personality and Social Psychology Bulletin, 18, 302-318.

*Lum, J. L. (1997). Ethnic differences in the expression of affection and other emotions. Unpublished doctoral dissertation, University of California, Santa Barbara.

Lyubomirsky, S. (1997, May). The meaning and expression of happiness: Comparing the United States and Russia. Paper presented at the annual meeting of the American Psychological Society, Washington, DC.

Lukes, S. (1973). Individualism. New York: Harper \& Row.

Ma, V., \& Schoeneman, T. J. (1997). Individualism versus collectivism: A comparison of Kenyan and American self-concepts. Basic and Applied Social Psychology, 19, 261-273.

Mallard, A. G. C., Lance, C. E., \& Michalos, A. C. (1997). Culture as a moderator of overall life satisfaction-Life facet satisfaction relationships. Social Indicators Research, 40, 259-284.

Mattick, R. P., \& Clarke, J. C. (1989). Development and validation of measures of social phobia scrutiny fear and social interaction anxiety. Behaviour Research and Therapy, 36, 455-470.

Markus, H. R., \& Kitayama, S. (1991). Culture and the self: Implications for cognition, emotion, and motivation. Psychological Review, 20, $568-$ 579.

Markus, H. R., \& Oyserman, D. (1989). Gender and thought: The role of the self-concept. In M. Crawford \& M. Gentry (Eds.), Gender and thought: Psychological perspectives (pp. 100-127). New York: Springer-Verlag.

Matsumoto, D. (1990). Cultural similarities and differences in display rules. Motivation and Emotion, 14, 195-214.

Matsumoto, D. (1999). Culture and self: An empirical assessment of Markus and Kitayama's theory of independent and interdependent selfconstruals. Asian Journal of Social Psychology, 2, 289-310.
*Matsumoto, D., Weissman, M. D., Preston, K., Brown, B. R., \& Kupperbusch, C. (1997). Context-specific measurement of individualismcollectivism on the individual level: The individualism-collectivism interpersonal assessment inventory. Journal of Cross-Cultural Psychology, 28, 743-767.

McCusker, C., \& Bottom, W. P. (2000). Non-structural solutions to freeriding in organizations: A transformational analysis. Unpublished manuscript, Yale University, New Haven, CT.

*McCusker, C., Nam, K., \& Chan, A. C.-F. (2000). A solution to the culture and negotiation puzzle: Individualism and collectivism in China, Korea, and the United States. Unpublished manuscript, Yale University, New Haven, CT.

Mehrabian, A. (1970). The development and validation of measures of affiliative tendency and sensitivity to rejection. Educational and Psychological Measurement, 20, 417-428.

Menon, T., Morris, M. W., Chiu, C., \& Hong, Y. (1999). Culture and the construal of agency: Attribution to individual versus group dispositions. Journal of Personality and Social Psychology, 76, 701-717.

Michalos, A. C. (1991). Global report of student well-being. New York: Springer.

Miller, J. G. (1984). Culture and the development of everyday social explanation. Journal of Personality and Social Psychology, 46, 961978.

Miller, J. G. (1986). Early cross-cultural commonalities in social explanation. Developmental Psychology, 22, 514-520.

Miller, J. G., \& Bersoff, D. M. (1992). Culture and moral judgment: How are conflicts between justice and interpersonal responsibilities resolved? Journal of Personality and Social Psychology, 62, 541-554.

Miller, J. G., \& Bersoff, D. M. (1994). Cultural influences on the moral status of reciprocity and the discounting of endogenous motivation. Personality and Social Psychology Bulletin, 20, 592-602.

Miller, J. G., \& Bersoff, D. M. (1998). The role of liking in perceptions of the moral responsibility to help: A cultural perspective. Journal of Experimental Social Psychology, 34, 443-469.

Miller, J. G., Bersoff, D. M., \& Harwood, R. L. (1990). Perceptions of social responsibilities in India and in the United States: Moral imperatives or personal decisions? Journal of Personality and Social Psychology, 58, 33-47.

Miller, J. G., \& Luthar, S. (1989). Issues of interpersonal responsibility and accountability: A comparison of Indians' and Americans' moral judgments. Social Cognition, 7, 237-261.

Mirowsky, J., \& Ross, C. E. (1991). Eliminating defense and agreement bias from measures of the sense of control: A $2 * 2$ index. Social Psychology Quarterly, 54, 127-145.

Modigliani, A. (1966). Embarrassability and social influence. Unpublished doctoral dissertation, University of Michigan, Ann Arbor.

Moorman, R. H., \& Blakely, G. L. (1995). Individualism-collectivism as an individual difference predictor of organizational citizenship behavior. Journal of Organizational Behavior, 16, 127-142.

Morris, M. W., \& Leung, K. (2000). Justice for all? Progress in research in cultural variation in the psychology of distributive and procedural justice. Applied Psychology: An International Review, 49, 100-132.

Morris, M. W., Nisbett, R. E., \& Peng, K. (1995). Causal attribution across domains and cultures. In D. Sperber, D. Premack, \& A. J. Premack (Eds.), Causal cognition: A multidisciplinary debate (pp. 577-613). Oxford, England: Oxford University Press.

Morris, M. W., \& Peng, K. (1994). Culture and cause: American and Chinese attributions for social and physical events. Journal of Personality and Social Psychology, 67, 949-971.

Newman, L. S. (1991). Why are traits inferred spontaneously? A developmental approach. Social Cognition, 9, 221-253.

Newman, L. S. (1993). How individualists interpret behavior: Idiocentrism and spontaneous trait inference. Social Cognition, 11, 243-269.

Nunnally, J. C. (1994). Psychometric theory. New York: McGraw-Hill. 
Oetzel, J. G. (1998a). Culturally homogeneous and heterogeneous groups: Explaining communication processes through individualismcollectivism and self-construal. International Journal of Intercultural Relations, 22, 135-161.

*Oetzel, J. G. (1998b). Explaining individual communication processes in homogeneous and heterogeneous groups through individualismcollectivism and self-construal. Human Communication Research, 25, 202-224.

Ohbuchi, K., Fukushima, O., \& Tedeschi, J. T. (1999). Cultural values in conflict management: Goal orientation, goal attainment, and tactical decision. Journal of Cross-Cultural Psychology, 30, 51-71.

*Oishi, S. (2000). Goals as cornerstones of subjective well-being: Linking individuals with cultures. In E. F. Diener \& E. M. Suh (Eds.), Subjective well-being across cultures (pp. 87-112). Cambridge, MA: MIT Press.

Oishi, S., Diener, E. F., Lucas, R. E., \& Suh, E. M. (1999). Cross-cultural variations in predictors of life satisfaction: Perspectives from needs and values. Personality and Social Psychology Bulletin, 25, 980-990.

*Oishi, S., Schimmack, U., Diener, E. F., \& Suh, E. M. (1998). The measurement of values and individualism-collectivism. Personality and Social Psychology Bulletin, 24, 1177-1189.

*Okazaki, S. (1997). Sources of ethnic differences between Asian American and White American college students on measures of depression and social anxiety. Journal of Abnormal Psychology, 106, 52-60.

*Okazaki, S. (2000). Asian American-White American difference on affective distress symptoms: Do symptom reports differ across reporting methods? Journal of Cross-Cultural Psychology, 31, 603-625.

Oyserman, D. (1993). The lens of personhood: Viewing the self, others, and conflict in a multicultural society. Journal of Personality and Social Psychology, 65, 993-1009.

*Oyserman, D., Gant, L., \& Ager, J. (1995). A socially contextualized model of African American identity: Possible selves and school persistence. Journal of Personality and Social Psychology, 69, 1216-1232.

*Oyserman, D., \& Lauffer, A. (in press). Examining the implications of cultural frames on social movements and group action. In L. Newman \& R. Erber (Eds.), What social psychology can tell us about the Holocaust. Oxford, England: Oxford University Press.

Oyserman, D., \& Markus, H. R. (1993). The sociocultural self. In J. Suls (Ed.), The self in social perspective (pp. 187-220). Hillsdale, NJ: Erlbaum.

Oyserman, D., Sakamoto, I., \& Lauffer, A. (1998). Cultural accommodation: Hybridity and the framing of social obligation. Journal of Personality and Social Psychology, 74, 1606-1618.

*Ozawa, K., Crosby, M., \& Crosby, F. (1996). Individualism and resistance to affirmative action: A comparison of Japanese and American samples. Journal of Applied Social Psychology, 26, 1138-1152.

Pam, A., Plutchik, R., \& Conte, H. R. (1975). Love: A psychometric approach. Psychological Reports, 37, 83-88.

Parks, C. D., \& Vu, A. D. (1994). Social dilemma behavior of individuals from highly individualist and collectivist cultures. Journal of Conflict Resolution, 38, 708-718.

Parks, M., \& Adelman, M. (1983). Communication networks and the development of romantic relationships: An expansion of uncertainty reduction theory. Human Communication Monographs, 10, 55-80.

Parsons, T., \& Shills, E. (1951). Toward a general theory of social action. Cambridge, MA: Harvard University Press.

*Pearson, V. M. S., \& Stephan, W. G. (1998). Preferences for styles of negotiation: A comparison of Brazil and the U.S. International Journal of Intercultural Relations, 22, 67-83.

Peng, K., Kemmelmeier, M., Burnstein, E., \& Manis, M. (1996, May). Individualism, collectivism and inter-ethnic friendships. Paper presented at the 60th anniversary conference of the Society on the Psychological Study of Social Issues, Ann Arbor, MI.

Peng, K., Nisbett, R. E., \& Wong, Y. C. (1997). Validity problems comparing values across cultures and possible solutions. Psychological Methods, 2, 329-344.

Perloe, S. I. (1967). Social values questionnaire. In J. P. Robinson \& P. R. Shaver (Eds.), Measures of social psychological attitudes (pp. 576585). Ann Arbor, MI: Survey Research Center, Institute for Social Research.

Pruitt, D. G., \& Lewis, S. A. (1975). Development of integrative solutions in bilateral negotiation. Journal of Personality and Social Psychology, 31, 621-633.

Putnam, L. L., \& Wilson, C. E. (1982). Communication strategies in organizational conflicts: Reliability and validity of a measurement. In M. Burgoon (Ed.), Communication yearbook (Vol. 6, pp. 629-652). Beverly Hills, CA: Sage.

Radloff, L. (1977). The CES-D Scale: A self-report depression scale for research in the general population. Applied Psychological Measurement, 1, 385-401.

Rahim, A. (1983). A measure of styles of handling interpersonal conflict. Academy of Management Journal, 26, 368-376.

Ramamoorthy, N., \& Carroll, S. J. (1998). Individualism/collectivism orientations and reactions toward alternative human resource management practices. Human Relations, 51, 571-588.

Raudenbush, S. W., Becker, B. J., \& Kalaian, S. (1988). Modeling multivariate effect sizes. Psychological Bulletin, 102, 111-120.

*Rhee, E., Uleman, J. S., \& Lee, H. K. (1996). Variations in collectivism and individualism by in-group and culture: Confirmatory factor analyses. Journal of Personality and Social Psychology, 71, 1037-1053.

Rhee, E., Uleman, J. S., Lee, H. K., \& Roman, R. J. (1995). Spontaneous self-descriptions and ethnic identities in individualistic and collectivistic cultures. Journal of Personality and Social Psychology, 69, 142-152.

*Robert, C. (1998). The development and validation of the vertical individualism and collectivism organizational culture scale. Unpublished doctoral dissertation, University of Illinois, Urbana-Champaign.

Rokeach, M. (1968). Beliefs, attitudes, and values: A theory of organization and change. San Francisco: Jossey-Bass.

*Rosen, C. F. (1997). Cultural dimensions of individualism-collectivism and power distance: Their influence on Vietnamese and Anglo-American undergraduates' conflict resolution preferences in developmental education. Unpublished doctoral dissertation, University of Minnesota, Minneapolis, MN.

Rosenberg, M. (1965). Society and the adolescent self-image. Princeton, NJ: Princeton University Press.

Ross, C. E., \& Mirowsky, J. (1984). Components of depressed mood in married men and women. The Center for Epidemiologic Studies Depression Scale. American Journal of Epidemiology, 119, 997-1004.

Rubin, Z. (1970). Measurement of romantic love. Journal of Personality and Social Psychology, 16, 265-273.

Rusbult, C. E., Martz, J. M., \& Agnew, C. R. (1998). The Investment Model Scale: Measuring commitment level, satisfaction level, quality of alternatives, and investment size. Personal Relationships, 5, 357-391.

Rusbult, C. E., Verette, J., Whitney, G. A., Slovik, L. F., \& Lipkus, I. (1991). Accommodation processes in close relationships: Theory and preliminary empirical evidence. Journal of Personality and Social Psychology, 60, 53-78.

Sampson, E. E. (1977). Psychology and the American ideal. Journal of Personality and Social Psychology, 35, 767-782.

Sampson, E. E. (1988). The debate on individualism: Indigenous psychologies of the individual and their role in personal and societal functioning. American Psychologist, 43, 15-22.

Sampson, E. E. (2001). Reinterpreting individualism and collectivism: Their religious roots and monologic versus dialogic person-other relationship. American Psychologist, 55, 1425-1432.

Sastry, J., \& Ross, C. E. (1998). Asian ethnicity and the sense of personal control. Social Psychology Quarterly, 61, 101-120.

*Sato, T., \& Cameron, J. E. (1999). The relationship between collective 
self-esteem and self-construal in Japan and Canada. Journal of Social Psychology, 139, 426-443.

Sato, T., \& McCann, D. (1998). Individual differences in relatedness and individuality: An exploration of two constructs. Personality and Individual Differences, 24, 847-859.

Sayle, M. (1998). Japan's social crisis. Atlantic Monthly, 281, 84-94.

Scherer, K. R. (1997). The role of culture in emotion-antecedent appraisal. Journal of Personality and Social Psychology, 73, 902-922.

Schwartz, S. H. (1990). Individualism-collectivism: Critique and proposed refinements. Journal of Cross-Cultural Psychology, 21, 139-157.

Schwartz, S. H. (1992). Universals in the content and structure of values: Theoretical advances and empirical tests in 20 countries. In M. P. Zanna (Ed.), Advances in experimental social psychology (Vol. 25, pp. 1-65). New York: Academic Press.

Schwartz, S. H. (1994). Beyond individualism/collectivism: New cultural dimensions of values. In U. Kim, H. C. Triandis, C. Kagitcibasi, S. Choi, \& G. Yoon (Eds.), Individualism and collectivism: Theory, method, and applications (pp. 85-119). Thousand Oaks, CA: Sage.

Sears, D. O. (1986). College sophomores in the laboratory: Influences of a narrow data base on social psychology's view of human nature. Journal of Personality and Social Psychology, 51, 515-530.

Seers, A. (1989). Team-member exchange quality: A new construct for role-making research. Organization Behavior and Human Decision Processes, 48, 118-135.

Sharkey, W. F., \& Singelis, T. M. (1995). Embarrassability and selfconstrual: A theoretical integration. Personality and Individual Differences, 19, 919-926.

*Shkodriani, G. M., \& Gibbons, J. L. (1995). Individualism and collectivism among university students in Mexico and the United States. Journal of Social Psychology, 135, 765-772.

Shweder, R. A., \& Bourne, E. J. (1982). Does the concept of the person vary cross-culturally? In A. J. Marsella \& G. M. White (Eds.), Cultural conceptions of mental health and therapy (pp. 97-137). New York: Reidel.

Simmons, C. H., vom Kolke, A., \& Shimizu, H. (1986). Attitudes toward romantic love among American, German, and Japanese students. Journal of Social Psychology, 126, 327-336.

*Singelis, T. M. (1994). The measurement of independent and interdependent self-construals. Personality and Social Psychology Bulletin, 20, $580-591$

*Singelis, T. M., Bond, M. H., Sharkey, W. F., \& Lai, C. S. Y. (1999). Unpackaging culture's influence on self-esteem and embarrassability. Journal of Cross-Cultural Psychology, 30, 315-341.

Singelis, T. M., \& Brown, W. J. (1995). Culture, self, and collectivist communication: Linking culture to individual behavior. Human Communication Research, 21, 354-389.

*Singelis, T. M., \& Sharkey, W. F. (1995). Culture, self-construal, and embarrassability. Journal of Cross-Cultural Psychology, 26, 622-644.

*Singelis, T. M., Triandis, H. C., Bhawuk, D. P. S., \& Gelfand, M. J. (1995). Horizontal and vertical dimensions of individualism and collectivism: A theoretical and measurement refinement. Cross-Cultural Research, 29, 240-275.

Sinha, D., \& Tripathi, R. C. (1994). Individualism in a collectivist culture: A case of coexistence of opposites. In U. Kim, H. C. Triandis, C. Kagitcibasi, S. Choi, \& G. Yoon (Eds.), Individualism and collectivism: Theory, method, and applications (pp. 123-136). Thousand Oaks, CA: Sage.

Smith, P. B., Dugan, S., Peterson, M. F., \& Leung, K. (1998). Individualism: Collectivism and the handling of disagreement. A 23-country study. International Journal of Intercultural Relations, 22, 351-367.

Snyder, C. R., \& Fromkin, H. L. (1977). Abnormality as a positive characteristic: The development and validation of a scale measuring need for uniqueness. Journal of Abnormal Psychology, 86, 518-527.
Stangor, C. (1998). Research methods for the behavioral sciences. Boston: Houghton Mifflin.

Steelman, L. M. (1995). Cultural influences upon altruism: Individualist vs. collectivist patterns of helpful responses with regard to causal attributions of need. Unpublished doctoral dissertation, University of Houston, TX.

Stein, C. H. (1992). Ties that bind: Three studies of obligation in adult relationships with family. Journal of Social and Personal Relationships, 9, 525-547.

*Stephan, C. W., Stephan, W. G., Saito, I., \& Barnett, S. M. (1998). Emotional expression in Japan and the United States: The nonmonolithic nature of individualism and collectivism. Journal of Cross-Cultural Psychology, 29, 728-748.

*Stephan, W. G., Stephan, C. W., \& de Vargas, M. C. (1996). Emotional expression in Costa Rica and the United States. Journal of CrossCultural Psychology, 27, 147-160.

Stipek, D. (1998). Differences between Americans and Chinese in the circumstances evoking pride, shame, and guilt. Journal of CrossCultural Psychology, 29, 616-629.

Suh, E., Diener, E., Oishi, S., \& Triandis, H. C. (1998). The shifting basis of life satisfaction judgments across cultures: Emotions versus norms. Journal of Personality and Social Psychology, 74, 482-493.

Tafarodi, R. W., \& Swann, W. B. (1996). Individualism-collectivism and global self-esteem: Evidence for a cultural trade-off. Journal of CrossCultural Psychology, 27, 651-672.

Takai, J., \& Ota, H. (1994). Assessing Japanese interpersonal communication competence. Japanese Journal of Experimental Social Psychology, 33, 224-236.

Takano, Y., \& Osaka, E. (1999). An unsupported common view: Comparing Japan and the U.S. on individualism/collectivism. Asian Journal of Social Psychology, 2, 311-341.

Takata, T. (1993). Social comparison and formation of self-concept in adolescence: Some findings about Japanese college students. Japanese Journal of Educational Psychology, 41, 339-348.

Thompson, L. L., \& Hastie, R. (1990). Social perception in negotiation. Organizational Behavior and Human Decision Processes, 47, 98-123.

Ting-Toomey, S. (1991). Intimacy expressions in three cultures: France, Japan, and the United States. International Journal of Intercultural Relations, 15, 29-46.

*Tong, A. K. K. (1996). A cross-cultural study of the perception, expression, regulation, and coping of emotion among Chinese and Caucasian people. Unpublished doctoral dissertation, University of Toronto, Toronto, Ontario, Canada.

Tönnies, F. (1957). Community and association (C. P. Loomis, Trans.). New York: Harper Torchbooks. (Original work published 1887)

Trafimow, D., Triandis, H. C., \& Goto, S. G. (1991). Some tests of the distinction between the private self and the collective self. Journal of Personality and Social Psychology, 60, 649-655.

*Trapnell, P. D. (1995). Mean differences in opinion conformity, and interdependent versus independent self-construal among European and Asian ancestry undergraduates. Unpublished manuscript, Ohio State University at Mansfield.

Triandis, H. C. (1989). The self and behavior in different cultural contexts. Psychological Review, 96, 506-520.

Triandis, H. C. (1991). Manual of instruments for the study of allocentrism or collectivism and idiocentrism or individualisms. Unpublished manuscript, University of Illinois, Urbana-Champaign.

Triandis, H. C. (1994). IND-COL. Unpublished research scale.

Triandis, H. C. (1995). Individualism and collectivism. Boulder, CO: Westview Press.

Triandis, H. C., Bontempo, R., Betancourt, H., Bond, M., Leung, K., Brenes, A., et al. (1986). The measurement of the etic aspects of individualism and collectivism across cultures. Australian Journal of Psychology, 38, 257-267. 
Triandis, H. C., Bontempo, R., Villareal, M. J., Asai, M., \& Lucca, N. (1988). Individualism and collectivism: Cross-cultural perspectives on self-ingroup relationships. Journal of Personality and Social Psychology, 54, 323-338.

Triandis, H. C., Chan, D. K., Bhawuk, D. P. S., Iwao, S., \& Sinha, J. B. P. (1995). Multimethod probes of allocentrism and idiocentrism. International Journal of Psychology, 4, 461-480.

Triandis, H. C., \& Gelfand, M. J. (1998). Converging measurement of horizontal and vertical individualism and collectivism. Journal of Personality and Social Psychology, 74, 118-128.

Triandis, H. C., Kurowski, L., \& Gelfand, M. J. (1994). Workplace diversity. In H. C. Triandis, M. D. Ounnette, \& L. M. Hough (Eds.), Handbook of industrial and organizational psychology (Vol. 4, pp. 769-827). Palo Alto, CA: Consulting Psychologists Press.

*Triandis, H. C., McCusker, C., Betancourt, H., Iwao, S., Leung, K., Salazar, J. M., et al. (1993). An etic-emic analysis of individualism and collectivism. Journal of Cross-Cultural Psychology, 24, 366-383.

Triandis, H. C., McCusker, C., \& Hui, C. H. (1990). Multimethod probes of individualism and collectivism. Journal of Personality and Social Psychology, 59, 1006-1020.

Trubinsky, P., Ting-Toomey, S., \& Lin, S. (1991). The influence of individualism-collectivism and self-monitoring on conflict styles. International Journal of Intercultural Relations, 15, 65-84.

Tziner, A., \& Elizur, D. (1985). Achievement motive: A reconceptualization and new instrument. Journal of Occupational Behavior, 6, 209228.

United Nations. (1994). 1992 Demographic Yearbook (Department of Economic and Social Information and Policy Analysis). New York: Author.

U.S. Department of Commerce. (2001). Profiles of general economic characteristics: 2000 Census of Population and Housing. Washington, DC: Author.

Vandello, J. A., \& Cohen, D. (1999). Patterns of individualism and collectivism across the United States. Journal of Personality and Social Psychology, 77, 279-292.

Vaux, A., Riedel, S., \& Stewart, D. (1987). Modes of social support: The Social Support Behavior (SS-B) scale. American Journal of Community Psychology, 15, 209-237.

Veenhoven, R. (1993). Happiness in nations. Rotterdam, the Netherlands: Risbo.

Verma, J. (1985). The ingroup and its relevance to individual behavior: A study of collectivism and individualism. Psychologia, 28, 173-181.

Verma, J. (1986). Perceived causes of norm violation as a function of individualism and collectivism. Psychological Studies, 31, 169-176.

Wagner, J. A. (1995). Studies of individualism-collectivism: Effects of cooperation in groups. Academy of Management Journal, 38, 152-172.

Wagner, J. A., \& Moch, M. K. (1986). Individualism-collectivism: Concept and measure. Group \& Organization Studies, 11, 1986, 280-304.

Waterman, A. S. (1984). The psychology of individualism. New York: Praeger.

Watkins, D., Akande, A., Fleming, J., Ismail, M., Lefner, K., Regmi, M., et al. (1998). Cultural dimensions, gender, and the nature of selfconcept: A fourteen-country study. International Journal of Psychology, 33, 17-31.
Watson, D., \& Friend, R. (1969). Measurement of social-evaluative anxiety. Journal of Consulting and Clinical Psychology, 33, 448-457.

Watson, D., Clark, L. A., \& Tellegen, A. (1988). Development and validation of brief measures of positive and negative affect: The PANAS scales. Journal of Personality and Social Psychology, 54, 1063-1070.

Weber, M. (1930). The Protestant ethic and the spirit of capitalism. New York: Routledge.

*Whatley, M. A. (1997, May). Exploratory factor analysis of an individualism-collectivism scale using an American sample. Paper presented at the annual meeting of the Midwestern Psychological Association, Chicago, IL.

Wheeler, L., Reis, H. T., \& Bond, M. H. (1989). Collectivismindividualism in everyday social life: The middle kingdom and the melting pot. Journal of Personality and Social Psychology, 57, 79-86.

Wink, P. (1997). Beyond ethnic differences: Contextualizing the influence of ethnicity on individualism and collectivism. Journal of Social Issues, 53, 329-349.

Wink, P., Gao, B., Jones, S., \& Chao, F. (1997). Social values and relationships with parents among American college women of Chinese and European descent. International Journal of Psychology, 32, 169179.

World Bank. (1994). World Development Report 1994: Infrastructure for development. Oxford, England: Oxford University Press.

World Values Survey Group. (1994). World Values Survey, 1981-1984 and 1990-1993 (ICPSR version) [Electronic data file]. Ann Arbor, MI: Institute for Social Research.

*Wosinska, W., Barrett, D. W., Butner, J., Cialdini, R. B., Edmondson, R., \& Kesler, M. (1999). Effects of individualism/collectivism on compliance in Mexico. Unpublished manuscript, Arizona State University, Tempe.

*Wu, S. M. (1995). Cross-cultural comparisons of values, attitudes, and behaviors within the context of an allocation game. Unpublished doctoral dissertation, Arizona State University, Tempe.

Yamagishi, T. (1988a). Exit from the group and in individualistic solution to the free rider problem in the United States and Japan. Journal of Experimental Social Psychology, 24, 530-542.

Yamagishi, T. (1988b). The provision of a sanctioning system in the United States and Japan. Social Psychology Quarterly, 51, 265-271.

Yamaguchi, S. (1994). Collectivism among the Japanese: A perspective from the self. In U. Kim, H. C. Triandis, C. Kagitcibasi, S. Choi, \& G. Yoon (Eds.), Individualism and collectivism: Theory, method, and applications (pp. 175-188). Thousand Oaks, CA: Sage.

Yamaguchi, S., Kuhlman, D. M., \& Sugimori, S. (1995). Personality correlates of allocentric tendencies in individualist and collectivist cultures. Journal of Cross-Cultural Psychology, 26, 658-672.

*Yang, N. (1996). Effects of individualism-collectivism on perceptions and outcomes of work-family conflict: A cross-cultural perspective. Unpublished doctoral dissertation, State University of New York, Buffalo.

Ybarra, O., \& Trafimow, D. (1998). How priming the private self or collective self affects the relative weights of attitudes and subjective norms. Personality and Social Psychology Bulletin, 24, 362-370.

Zimmerman, M., \& Coryell, W. (1987). The Inventory to Diagnose Depression (IDD): A self-report scale to diagnose major depressive disorder. Journal of Consulting and Clinical Psychology, 55, 55-59. 
Appendix A

Studies Used in International Meta-Analyses

\begin{tabular}{|c|c|c|c|c|c|c|c|c|c|}
\hline \multirow[b]{2}{*}{ Study } & \multirow{2}{*}{$\begin{array}{l}\text { Comparison } \\
\text { countries }(n)\end{array}$} & \multirow[b]{2}{*}{$\begin{array}{c}\text { North } \\
\text { America }(n)\end{array}$} & \multirow{2}{*}{$\begin{array}{l}\text { Overall \% } \\
\text { female }^{\mathrm{a}}\end{array}$} & \multirow[b]{2}{*}{ Population } & \multirow[b]{2}{*}{ IND/COL measure } & \multicolumn{2}{|c|}{ Effect size $(d)$} & \multicolumn{2}{|c|}{ Reliability } \\
\hline & & & & & & IND & $\mathrm{COL}$ & IND & $\mathrm{COL}$ \\
\hline Arikawa \& Templer (1998) & Japan (50) & 63 & $20(62)$ & CS & COL: Yamaguchi (1994) & & 0.81 & & - \\
\hline $\begin{array}{l}\text { Bloom, Oyserman, Menard, } \\
\text { \& Masataka }(2000)^{\mathrm{b}}\end{array}$ & Japan (68) & 41 & 100 & $\mathrm{AD}$ & $\begin{array}{l}\text { IND \& COL: Oyserman \& Lauffer } \\
\text { (in press) }\end{array}$ & 0.09 & -0.59 & low & low \\
\hline Brett \& Okumura (1998) & Japan (66) & 124 & - & MNG & IND: Schwartz (1994) & 0.66 & & high & \\
\hline Carter \& Dinnel (1997) & Japan (94) & 105 & $51(50)$ & CS & $\begin{array}{l}\text { IND: Singelis (1994) } \\
\text { COL: Singelis (1994), Triandis et al. } \\
\quad \text { (1990), Yamaguchi (1994) }\end{array}$ & -0.12 & 0.28 & low & low \\
\hline $\begin{array}{l}\text { Chen, Brockner, \& Katz } \\
\text { (1998) }\end{array}$ & China (80) & 292 & - & CS & $\begin{array}{l}\text { IND: Singelis et al. (1995), Triandis } \\
\text { et al. (1986) } \\
\text { COL: Singelis (1994), Singelis et al. } \\
\text { (1995) }\end{array}$ & 0.76 & -0.79 & - & - \\
\hline Chen, Meindl, \& Hui (1998) & Hong Kong (126) & 115 & $29(69)$ & $\mathrm{AD}$ & COL: Hui (1988) & & -0.18 & & low \\
\hline Chew (1996) & $\begin{array}{l}\text { Germany }(100) \\
\text { Puerto Rico (146) } \\
\text { Singapore }(220)\end{array}$ & 251 & 61 & CS & IND: Chew (1996) & $\begin{array}{r}-0.72 \\
-0.34 \\
0.16\end{array}$ & & $\begin{array}{l}\text { high } \\
\text { high } \\
\text { high }\end{array}$ & \\
\hline Cialdini et al. (1999) & Poland (270) & 235 & $60(73)$ & CS & $\begin{array}{l}\text { COL: Bierbrauer, Heyer, \& Wolfradt } \\
\text { (1994) }\end{array}$ & & -0.54 & & low \\
\hline $\begin{array}{l}\text { Cocroft \& Ting-Toomey } \\
\text { (1994) }\end{array}$ & Japan (190) & 197 & $43(59)$ & CS & $\begin{array}{l}\text { IND \& COL: Hamaguchi (1977), as } \\
\text { reported by Befu (1990); Triandis } \\
\text { et al. (1986) }\end{array}$ & -1.89 & -0.72 & low & low \\
\hline Dinnel \& Kleinknecht (1999) & Japan (142) & 123 & $55(67)$ & CS & IND \& COL: Singelis (1994) & 0.50 & 0.02 & low & low \\
\hline Earley (1989) & PR China (48) & 48 & $29(19)$ & MNT & COL: Erez \& Earley (1987) & & -1.93 & & high \\
\hline Earley (1993) & $\begin{array}{l}\text { PR China (60), } \\
\text { Israel (45) }\end{array}$ & 60 & $\begin{array}{l}13(22) \\
11\end{array}$ & MNG & $\begin{array}{l}\text { COL: Erez \& Earley (1987), } \\
\text { Triandis et al. (1986) }\end{array}$ & & $\begin{array}{l}-1.13 \\
-1.21\end{array}$ & & $\begin{array}{l}\text { high } \\
\text { low }\end{array}$ \\
\hline Earley (1994, Study 1) & $\begin{array}{l}\text { China (96) } \\
\text { Hong Kong (67) }\end{array}$ & 87 & $\begin{array}{l}25(29) \\
30\end{array}$ & MNT & COL: Earley (1993) & & $\begin{array}{l}-1.45 \\
-1.27\end{array}$ & & $\begin{array}{l}\text { high } \\
\text { high }\end{array}$ \\
\hline Earley (1994, Study 2) & PR China (46) & 62 & $43(31)$ & MNT & COL: Earley (1993) & & -0.71 & & high \\
\hline Erez \& Earley (1987) & Israel (120) & 60 & - & CS & COL: Erez \& Earley (1987) & & -0.84 & & low \\
\hline Gardner et al. (1999, Study 2) & Hong Kong (82) & 75 & $60(51)$ & CS & $\begin{array}{l}\text { IND \& COL: Schwartz (1994), } \\
\text { Triandis et al. (1990) }\end{array}$ & 0.34 & 0.34 & low & high \\
\hline Gire $(1997)^{\mathrm{b}}$ & Nigeria (90) & 95 & - & CS & COL: Hui (1988) & & -0.70 & & low \\
\hline Gire \& Carment $(1993)^{\mathrm{b}}$ & Nigeria (120) & 120 & - & CS & COL: Hui (1988) & & -0.06 & & - \\
\hline $\begin{array}{l}\text { Grimm, Church, Katigbak, } \\
\text { \& Reyes (1999) }\end{array}$ & Philippines (656) & 660 & 57 & $\begin{array}{l}\text { CS, HS } \\
\text { U.S.: CS }\end{array}$ & COL: Hui (1988) & & -0.05 & & high \\
\hline Gudykunst et al. (1996) & $\begin{array}{l}\text { Australia (110) } \\
\text { Japan }(192) \\
\text { Korea }(168)\end{array}$ & 283 & $\begin{array}{l}70(57) \\
45 \\
23\end{array}$ & CS & $\begin{array}{l}\text { IND \& COL: Gudykunst et al. } \\
\text { (1996) }\end{array}$ & $\begin{array}{r}0.25 \\
0.08 \\
-0.17\end{array}$ & $\begin{array}{l}-0.04 \\
-0.17 \\
-0.36\end{array}$ & $\begin{array}{l}\text { high } \\
\text { high } \\
\text { high }\end{array}$ & $\begin{array}{l}\text { high } \\
\text { high } \\
\text { high }\end{array}$ \\
\hline Hui (1988) & Hong Kong (108) & 132 & 50 & CS & COL: Hui (1988) & & 0.72 & & - \\
\hline Hui, Triandis, \& Yee (1991) & Hong Kong (72) & 88 & - & CS & COL: Hui (1988) & & 0.61 & & - \\
\hline Kashima et al. (1995) & $\begin{array}{l}\text { Australia (158) } \\
\text { Japan }(256) \\
\text { Korea }(254)\end{array}$ & 134 & $\begin{array}{l}77(48) \\
52 \\
69\end{array}$ & CS & $\begin{array}{l}\text { IND: Yamaguchi (1994); COL: } \\
\text { Hamaguchi (1977), Triandis et al. } \\
\text { (1986) }\end{array}$ & $\begin{array}{r}-0.15 \\
0.88 \\
0.93\end{array}$ & $\begin{array}{r}-0.01 \\
0.24 \\
0.11\end{array}$ & $\begin{array}{l}\text { high } \\
\text { high } \\
\text { high }\end{array}$ & $\begin{array}{l}\text { high } \\
\text { high } \\
\text { high }\end{array}$ \\
\hline Kemmelmeier et al. (2001) & $\begin{array}{l}\text { Bulgaria (322) } \\
\text { Germany }(102) \\
\text { Japan }(85) \\
\text { N. Zealand (111) } \\
\text { Poland (109) }\end{array}$ & 192 & $\begin{array}{l}77(57) \\
78 \\
48 \\
51 \\
44\end{array}$ & CS & IND \& COL: Singelis et al. (1995) & $\begin{array}{r}-0.41 \\
0.39 \\
0.96 \\
-0.22 \\
0.00\end{array}$ & $\begin{array}{r}-0.06 \\
1.04 \\
0.73 \\
0.54 \\
0.20\end{array}$ & $\begin{array}{l}\text { low } \\
\text { low } \\
\text { low } \\
\text { low } \\
\text { low }\end{array}$ & $\begin{array}{l}\text { low } \\
\text { low } \\
\text { low } \\
\text { low } \\
\text { low }\end{array}$ \\
\hline $\begin{array}{l}\text { M. Kim, Hunter, Miyahara, } \\
\text { \& Horvath (1996) }\end{array}$ & $\begin{array}{l}\text { Japan }(199) \\
\text { Korea }(290)\end{array}$ & 239 & $\begin{array}{l}17(28) \\
25\end{array}$ & CS & $\begin{array}{l}\text { IND \& COL: Breckler, Greenwald, } \\
\text { \& Wiggins (1986) }\end{array}$ & $\begin{array}{l}0.96 \\
0.43\end{array}$ & $\begin{array}{r}-0.01 \\
0.18\end{array}$ & $\begin{array}{l}\text { high } \\
\text { low }\end{array}$ & $\begin{array}{l}\text { low } \\
\text { low }\end{array}$ \\
\hline Kleinknecht et al. (1997) & Japan (161) & 181 & $55(64)$ & CS & IND \& COL: Singelis (1994) & 0.00 & 0.29 & low & low \\
\hline Krull et al. (1999, Study 1) & Taiwan (60) & 36 & $47(58)$ & CS & IND \& COL: Singelis (1994) & 0.24 & 0.69 & - & - \\
\hline Krull et al. (1999, Study 2) & Hong Kong (38) & 38 & 50 & CS & $\begin{array}{l}\text { IND \& COL: Gudykunst et al. } \\
\text { (1996) }\end{array}$ & 0.93 & 0.59 & - & - \\
\hline
\end{tabular}


Appendix A (continued)

\begin{tabular}{|c|c|c|c|c|c|c|c|c|c|}
\hline \multirow[b]{2}{*}{ Study } & \multirow[b]{2}{*}{$\begin{array}{l}\text { Comparison } \\
\text { countries }(n)\end{array}$} & \multirow[b]{2}{*}{$\begin{array}{c}\text { North } \\
\text { America }(n)\end{array}$} & \multirow[b]{2}{*}{$\begin{array}{l}\text { Overall \% } \\
\text { female }^{\mathrm{a}}\end{array}$} & \multirow[b]{2}{*}{ Population } & \multirow[b]{2}{*}{ IND/COL measure } & \multicolumn{2}{|c|}{ Effect size $(d)$} & \multicolumn{2}{|c|}{ Reliability } \\
\hline & & & & & & IND & $\mathrm{COL}$ & IND & $\mathrm{COL}$ \\
\hline $\begin{array}{l}\text { Kwan, Bond, \& Singelis } \\
\text { (1997) }\end{array}$ & Hong Kong (194) & 183 & $68(58)$ & CS & IND \& COL: Singelis (1994) & 0.37 & -0.83 & low & low \\
\hline Leung \& Iwawaki (1998) & Japan (160) & 164 & $50(49)$ & CS & COL: Hui (1988; two subscales) & & 0.03 & & low \\
\hline Liang (1997) & China (64) & 54 & 50 & GS & IND \& COL: Triandis et al. (1990) & -0.40 & -0.24 & low & high \\
\hline $\begin{array}{l}\text { Matsumoto et al. (1997, } \\
\text { Study 6) }\end{array}$ & $\begin{array}{l}\text { Japan }(120) \\
\text { Russia (50) } \\
\text { Korea (71) }\end{array}$ & 285 & $\begin{array}{l}50(73) \\
82 \\
49\end{array}$ & CS & COL: Matsumoto et al. (1997) & & $\begin{array}{r}.05 \\
-0.94 \\
-0.32\end{array}$ & & - \\
\hline $\begin{array}{l}\text { McCusker, Nam, \& Chan } \\
\text { (2000) }\end{array}$ & Korea $(60)$ & 60 & - & CS & $\begin{array}{l}\text { COL: Triandis, Kurowski, \& } \\
\text { Gelfand (1994; } 5 \text { items) }\end{array}$ & & -1.74 & low & low \\
\hline Oetzel (1998b) & Japan (62) & 86 & $56(53)$ & CS & $\begin{array}{l}\text { IND \& COL: Gudykunst et al. } \\
\text { (1996) }\end{array}$ & 1.03 & 0.12 & high & low \\
\hline Oishi (2000) & $\begin{array}{l}\text { Argentina (90) } \\
\text { Australia (292) } \\
\text { Austria (164) } \\
\text { Bahrain (124) } \\
\text { Brazil (112) } \\
\text { Colombia (100) } \\
\text { Denmark (91) } \\
\text { Egypt (120) } \\
\text { Estonia (119) } \\
\text { Finland (91) } \\
\text { Germany (108) } \\
\text { Ghana (118) } \\
\text { Greece (129) } \\
\text { Guam (186) } \\
\text { Hong Kong (142) } \\
\text { Hungary (74) } \\
\text { India (93) } \\
\text { Indonesia (90) } \\
\text { Italy (289) } \\
\text { Japan (290) } \\
\text { Korea (277) } \\
\text { Lithuania (101) } \\
\text { Nepal (99) } \\
\text { Nigeria (244) } \\
\text { Norway (99) } \\
\text { Pakistan (155) } \\
\text { Peru (129) } \\
\text { Portugal (139) } \\
\text { PR China (558) } \\
\text { Puerto Rico (87) } \\
\text { Singapore (131) } \\
\text { Slovenia (50) } \\
\text { South Africa (373) } \\
\text { Spain (327) } \\
\text { Taiwan (533) } \\
\text { Tanzania (96) } \\
\text { Turkey (100) } \\
\text { Zimbabwe (109) }\end{array}$ & 443 & $\begin{array}{l}80(48) \\
81 \\
60 \\
60 \\
46 \\
92 \\
78 \\
48 \\
68 \\
79 \\
79 \\
47 \\
75 \\
66 \\
80 \\
69 \\
31 \\
51 \\
67 \\
65 \\
42 \\
74 \\
45 \\
35 \\
81 \\
69 \\
87 \\
67 \\
62 \\
24 \\
85 \\
62 \\
68 \\
85 \\
71 \\
35 \\
54 \\
63\end{array}$ & CS & 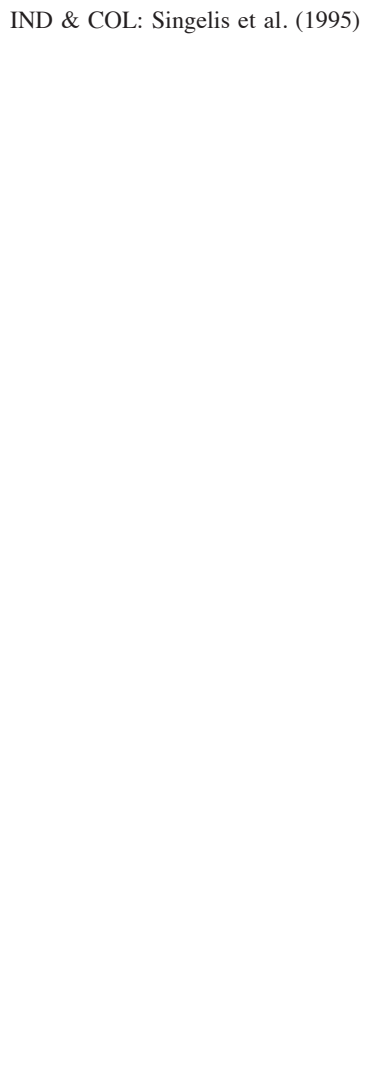 & $\begin{array}{r}-0.25 \\
-0.01 \\
-0.42 \\
0.52 \\
0.34 \\
-0.21 \\
0.40 \\
1.35 \\
0.23 \\
-0.04 \\
0.29 \\
0.55 \\
-0.01 \\
0.04 \\
0.94 \\
0.70 \\
-0.01 \\
0.04 \\
-0.02 \\
0.76 \\
0.64 \\
-0.48 \\
0.28 \\
0.03 \\
0.10 \\
0.15 \\
-0.40 \\
0.29 \\
0.66 \\
-0.28 \\
0.34 \\
-0.08 \\
-0.19 \\
0.53 \\
0.82 \\
0.83 \\
-0.03 \\
0.55\end{array}$ & $\begin{array}{r}-0.74 \\
0.11 \\
-0.03 \\
-0.88 \\
-0.60 \\
-0.81 \\
-0.28 \\
0.72 \\
-0.09 \\
-0.02 \\
-0.62 \\
-1.01 \\
-0.42 \\
-0.39 \\
0.60 \\
-0.36 \\
-0.60 \\
-0.32 \\
-1.17 \\
0.29 \\
0.17 \\
-0.24 \\
-0.80 \\
-1.48 \\
-0.07 \\
-1.47 \\
-1.83 \\
-1.12 \\
-0.97 \\
-0.65 \\
0.09 \\
-0.37 \\
-0.43 \\
-0.65 \\
-1.46 \\
0.28 \\
-0.66 \\
-1.00\end{array}$ & $\begin{array}{l}\text { low } \\
\text { high } \\
\text { low } \\
\text { low } \\
\text { low } \\
\text { high } \\
\text { low } \\
\text { high } \\
\text { low } \\
\text { high } \\
\text { high } \\
\text { low } \\
\text { low } \\
\text { high } \\
\text { high } \\
\text { high } \\
\text { low } \\
\text { high } \\
\text { low } \\
\text { low } \\
\text { low } \\
\text { low } \\
\text { high } \\
\text { low } \\
\text { low } \\
\text { low } \\
\text { high } \\
\text { low } \\
\text { high } \\
\text { low } \\
\text { low } \\
\text { low } \\
\text { low } \\
\text { low } \\
\text { low } \\
\text { high } \\
\text { high } \\
\text { high }\end{array}$ & $\begin{array}{l}\text { low } \\
\text { low } \\
\text { low } \\
\text { low } \\
\text { low } \\
\text { low } \\
\text { low } \\
\text { low } \\
\text { low } \\
\text { low } \\
\text { low } \\
\text { low } \\
\text { low } \\
\text { low } \\
\text { low } \\
\text { low } \\
\text { low } \\
\text { low } \\
\text { low } \\
\text { low } \\
\text { low } \\
\text { low } \\
\text { low } \\
\text { low } \\
\text { low } \\
\text { low } \\
\text { low } \\
\text { low } \\
\text { low } \\
\text { low } \\
\text { low } \\
\text { low } \\
\text { low } \\
\text { low } \\
\text { low } \\
\text { low } \\
\text { low } \\
\text { low }\end{array}$ \\
\hline Pearson \& Stephan (1998) & Brazil (200) & 219 & $50(54)$ & CS & COL: Triandis et al. (1988) & & 0.19 & & high \\
\hline Rhee, Uleman, \& Lee (1996) & Korea (220) & 133 & $30(59)$ & $\mathrm{CS}$ & $\begin{array}{l}\text { IND \& COL: Hui (1988), Triandis } \\
\text { (1991), Yamaguchi (1994) }\end{array}$ & 0.03 & -0.08 & - & - \\
\hline Robert (1998) & $\begin{array}{l}\text { India (183) } \\
\text { Mexico (252) } \\
\text { Poland (245) }\end{array}$ & 238 & $\begin{array}{l}5(39) \\
27 \\
29\end{array}$ & $\mathrm{AD}$ & IND \& COL: Singelis et al. (1995) & $\begin{array}{l}0.78 \\
0.52 \\
0.17\end{array}$ & $\begin{array}{l}-0.26 \\
-0.29 \\
-0.27\end{array}$ & $\begin{array}{l}\text { low } \\
\text { low } \\
\text { high }\end{array}$ & $\begin{array}{l}\text { low } \\
\text { low } \\
\text { low }\end{array}$ \\
\hline Rosen (1997) & Vietnam (101) & 104 & $56(58)$ & $\mathrm{CS}$ & IND \& COL: Singelis et al. (1995) & 0.67 & -0.19 & high & low \\
\hline Sato \& Cameron (1999) ${ }^{\mathrm{b}}$ & Japan (120) & 172 & 49 (Canada, 78) & CS & IND \& COL: Singelis (1994) & 0.04 & 0.72 & $\begin{array}{l}\text { low } \\
\text { high }\end{array}$ & $\begin{array}{l}\text { high } \\
\text { high }\end{array}$ \\
\hline Shkodriani \& Gibbons (1995) & Mexico (79) & 124 & - & CS & COL: Hui (1988) & & 1.33 & & - \\
\hline Singelis et al. (1999) & Hong Kong (271) & 232 & $58(38)$ & CS & IND \& COL: Singelis (1994) & 0.87 & 0.18 & low & low \\
\hline $\begin{array}{l}\text { Stephan, Stephan, Saito, \& } \\
\text { Barnett (1998) }\end{array}$ & Japan (100) & 100 & 50 & $\mathrm{CS}$ & IND \& COL: Triandis et al. (1988) & -0.06 & -0.91 & low & low \\
\hline $\begin{array}{l}\text { Stephan, Stephan, \& de } \\
\quad \text { Vargas (1996) }\end{array}$ & Costa Rica (68) & 102 & $58(38)$ & CS & IND \& COL: Kitayama et al. (1991) & 0.50 & 0.26 & low & low \\
\hline
\end{tabular}


Appendix A (continued)

\begin{tabular}{|c|c|c|c|c|c|c|c|c|c|}
\hline \multirow[b]{2}{*}{ Study } & \multirow[b]{2}{*}{$\begin{array}{l}\text { Comparison } \\
\text { countries }(n)\end{array}$} & \multirow[b]{2}{*}{$\begin{array}{c}\text { North } \\
\text { America }(n)\end{array}$} & \multirow[b]{2}{*}{$\begin{array}{l}\text { Overall } \% \\
\text { female }^{\mathrm{a}}\end{array}$} & \multirow[b]{2}{*}{ Population } & \multirow[b]{2}{*}{ IND/COL measure } & \multicolumn{2}{|c|}{ Effect size $(d)$} & \multicolumn{2}{|c|}{ Reliability } \\
\hline & & & & & & IND & $\mathrm{COL}$ & IND & $\mathrm{COL}$ \\
\hline Tong (1996) & Hong Kong (136) & 91 & $44(55)$ & CS & IND \& COL: Singelis et al. (1995) & 0.43 & 0.01 & - & - \\
\hline Trapnell (1995) & Hong Kong (167) & 214 & $64(66)$ & CS & IND \& COL: Singelis (1994) & 0.45 & -0.41 & high & low \\
\hline Triandis et al. (1993) & $\begin{array}{l}\text { Chile (141) } \\
\text { France (200) } \\
\text { Hong Kong (189) } \\
\text { India (109) } \\
\text { Indonesia (200) } \\
\text { Japan (78) } \\
\text { Poland (200) } \\
\text { PR China (200) } \\
\text { Venezuela (99) }\end{array}$ & 198 & 50 & $\mathrm{CS}$ & IND \& COL: Triandis et al. (1986) & $\begin{array}{r}-0.31 \\
0.47 \\
0.83 \\
-0.07 \\
0.12 \\
-0.70 \\
0.28 \\
0.01 \\
0.58\end{array}$ & $\begin{array}{c}-0.45 \\
0.03 \\
-0.61 \\
-0.52 \\
0.29 \\
-0.189 \\
0.32 \\
-0.41 \\
0.39\end{array}$ & $\begin{array}{l}\text { high } \\
\text { high } \\
\text { high } \\
\text { high } \\
\text { high } \\
\text { high } \\
\text { high } \\
\text { high } \\
\text { high }\end{array}$ & $\begin{array}{l}\text { high } \\
\text { high } \\
\text { high } \\
\text { high } \\
\text { high } \\
\text { high } \\
\text { high } \\
\text { high } \\
\text { high }\end{array}$ \\
\hline Wosinska et al. (1999) & Mexico (175) & 235 & $67(73)$ & $\mathrm{CS}$ & COL: Bierbrauer et al. (1994) & & -1.53 & & low \\
\hline Wu (1995) & Taiwan (90) & 90 & $56(64)$ & $\mathrm{CS}$ & $\begin{array}{l}\text { COL: Schwartz (1994), Triandis et } \\
\text { al. (1986) }\end{array}$ & & -0.13 & & low \\
\hline Yang (1996) & China (192) & 254 & $56(58)$ & $\mathrm{AD}$ & $\begin{array}{l}\text { COL: Hui (1988), Wagner \& Moch } \\
\text { (1986) }\end{array}$ & & 0.18 & & low \\
\hline
\end{tabular}

Note. Dashes indicate value is not available. IND = individualism; $\mathrm{COL}=$ collectivism; $\mathrm{CS}=$ college students $; \mathrm{AD}=$ adults; $\mathrm{MNG}=$ managers; $\mathrm{MNT}=$ manager trainees; HS = high school students; GS = graduate students; N. Zealand = New Zealand; PR China = People's Republic of China.

a Number in parentheses in this column is the overall percentage of U.S. participants. ${ }^{\mathrm{b}}$ This study used a Canadian comparison sample. 


\section{Appendix B}

Studies Used in Within-U.S. Meta-Analyses

\begin{tabular}{|c|c|c|c|c|c|c|c|c|c|}
\hline \multirow[b]{2}{*}{ Study } & \multirow{2}{*}{$\begin{array}{l}\text { Comparison } \\
\text { groups }(n)\end{array}$} & \multirow{2}{*}{$\begin{array}{c}\text { European } \\
\text { American }(n)\end{array}$} & \multirow{2}{*}{$\begin{array}{l}\text { Overall \% } \\
\text { female }^{\mathrm{a}}\end{array}$} & \multirow[b]{2}{*}{ Population } & \multirow[b]{2}{*}{ IND/COL measure } & \multicolumn{2}{|c|}{ Effect size $(d)$} & \multicolumn{2}{|c|}{ Reliability } \\
\hline & & & & & & IND & $\mathrm{COL}$ & IND & $\mathrm{COL}$ \\
\hline Benet-Martinez (2000) & $\begin{array}{l}\text { AsA (205) } \\
\text { AfA (30) } \\
\text { LtA (77) }\end{array}$ & 122 & $\begin{array}{l}62(55) \\
80 \\
70\end{array}$ & CS & IND \& COL: Singelis et al. (1995) & $\begin{array}{r}0.06 \\
-0.55 \\
-0.30\end{array}$ & $\begin{array}{r}-0.47 \\
0.00 \\
-0.31\end{array}$ & $\begin{array}{l}\text { low } \\
\text { low } \\
\text { low }\end{array}$ & $\begin{array}{l}\text { low } \\
\text { low } \\
\text { low }\end{array}$ \\
\hline Carpenter \& Radhakrishnan (1998a) & LtA (42) & 34 & $52(55)$ & CS & IND \& COL: Singelis et al. (1995) & 0.45 & -0.21 & high & high \\
\hline Carpenter \& Radhakrishnan (1998b) & LtA (126) & 132 & $58(55)$ & CS & IND \& COL: Singelis et al. (1995) & 0.07 & -0.08 & high & high \\
\hline Carpenter \& Radhakrishnan (1999) & LtA (65) & 66 & $62(59)$ & CS & IND \& COL: Singelis et al. (1995) & 0.21 & 0.06 & high & high \\
\hline $\begin{array}{l}\text { Coon \& Kemmelmeier ( } 2001, \\
\text { Sample 1) }\end{array}$ & $\begin{array}{l}\text { AfA (29) } \\
\text { AsA (29) } \\
\text { LtA (21) }\end{array}$ & 305 & $\begin{array}{l}47(53) \\
44 \\
46\end{array}$ & CS & IND \& COL: Singelis (1994) & $\begin{array}{r}-0.46 \\
0.18 \\
0.15\end{array}$ & $\begin{array}{r}-0.07 \\
-0.36 \\
0.12\end{array}$ & $\begin{array}{l}\text { low } \\
\text { high } \\
\text { low }\end{array}$ & $\begin{array}{l}\text { low } \\
\text { low } \\
\text { high }\end{array}$ \\
\hline $\begin{array}{l}\text { Coon \& Kemmelmeier (2001, } \\
\text { Sample 2) }\end{array}$ & $\begin{array}{l}\text { AfA (9) } \\
\text { AsA (22) } \\
\text { LtA (6) }\end{array}$ & 110 & $\begin{array}{l}36(54) \\
44 \\
50\end{array}$ & CS & IND \& COL: Singelis (1994) & $\begin{array}{r}-0.49 \\
-0.11 \\
0.22\end{array}$ & $\begin{array}{r}0.51 \\
-0.29 \\
-0.01\end{array}$ & $\begin{array}{l}\text { low } \\
\text { high } \\
\text { low }\end{array}$ & $\begin{array}{l}\text { low } \\
\text { high } \\
\text { low }\end{array}$ \\
\hline $\begin{array}{l}\text { Coon \& Kemmelmeier ( } 2001, \\
\text { Sample 3) }\end{array}$ & $\begin{array}{l}\text { AfA (29) } \\
\text { AsA (40) } \\
\text { LtA (9) }\end{array}$ & 238 & $\begin{array}{l}45(58) \\
59 \\
11\end{array}$ & CS & IND \& COL: Singelis (1994) & $\begin{array}{r}-0.28 \\
0.12 \\
-0.13\end{array}$ & $\begin{array}{r}0.12 \\
-0.32 \\
0.60\end{array}$ & $\begin{array}{l}\text { low } \\
\text { low } \\
\text { low }\end{array}$ & $\begin{array}{l}\text { low } \\
\text { low } \\
\text { high }\end{array}$ \\
\hline $\begin{array}{l}\text { Coon \& Kemmelmeier (2001, } \\
\text { Sample 4) }\end{array}$ & $\begin{array}{l}\text { AfA (23) } \\
\text { AsA (18) } \\
\text { LtA (7) }\end{array}$ & 122 & $\begin{array}{l}61(69) \\
61 \\
57\end{array}$ & CS & IND \& COL: Singelis et al. (1995) & $\begin{array}{l}-0.62 \\
-0.06 \\
-0.46\end{array}$ & $\begin{array}{r}0.02 \\
-0.34 \\
-0.29\end{array}$ & $\begin{array}{l}\text { low } \\
\text { low } \\
\text { low }\end{array}$ & $\begin{array}{l}\text { low } \\
\text { high } \\
\text { high }\end{array}$ \\
\hline $\begin{array}{l}\text { Coon \& Kemmelmeier ( } 2001, \\
\text { Sample 5) }\end{array}$ & $\begin{array}{l}\text { AfA (129) } \\
\text { AsA (50) } \\
\text { LtA (4) }\end{array}$ & 311 & $\begin{array}{l}58(55) \\
65 \\
75\end{array}$ & CS & $\begin{array}{l}\text { IND \& COL: Oyserman \& Lauffer } \\
\text { (in press) }\end{array}$ & $\begin{array}{r}-0.27 \\
0.13 \\
-0.13\end{array}$ & $\begin{array}{r}0.12 \\
-0.32 \\
0.61\end{array}$ & $\begin{array}{l}\text { high } \\
\text { high } \\
\text { high }\end{array}$ & $\begin{array}{l}\text { high } \\
\text { high } \\
\text { high }\end{array}$ \\
\hline Ebreo (1998) & $\begin{array}{l}\text { AfA (84) } \\
\text { AsA (58) } \\
\text { LtA (51) }\end{array}$ & 109 & $\begin{array}{l}60(59) \\
70 \\
53\end{array}$ & CS & COL: Triandis et al. (1988) & & $\begin{array}{r}0.02 \\
-0.25 \\
-0.17\end{array}$ & & $\begin{array}{l}\text { high } \\
\text { high } \\
\text { high }\end{array}$ \\
\hline Freeberg \& Stein (1996) & LtA (50) & 50 & 50 & $\begin{array}{l}\text { young } \\
\text { adults }\end{array}$ & IND \& COL: Yamaguchi (1994) & -0.03 & -0.45 & low & low \\
\hline $\begin{array}{l}\text { Gaines et al. (1997, Study 1, } \\
\text { Sample 2) }\end{array}$ & $\begin{array}{l}\text { AfA (5) } \\
\text { AsA (14) } \\
\text { LtA (12) }\end{array}$ & 31 & 62 & GS & IND \& COL: Gaines et al. (1997) & $\begin{array}{r}0.78 \\
-0.28 \\
1.01\end{array}$ & $\begin{array}{l}-1.28 \\
-0.71 \\
-0.74\end{array}$ & $\begin{array}{l}\text { low } \\
\text { low } \\
\text { low }\end{array}$ & $\begin{array}{l}\text { high } \\
\text { high } \\
\text { high }\end{array}$ \\
\hline $\begin{array}{l}\text { Gaines et al. (1997, Study 1, } \\
\text { Sample 6) }\end{array}$ & $\begin{array}{l}\text { AfA (18) } \\
\text { AsA (5) } \\
\text { LtA (3) }\end{array}$ & 24 & 60 & CS & IND \& COL: Gaines et al. (1997) & $\begin{array}{r}-0.08 \\
0.08 \\
-0.91\end{array}$ & $\begin{array}{l}-0.79 \\
-0.61 \\
-0.19\end{array}$ & $\begin{array}{l}\text { low } \\
\text { low } \\
\text { low }\end{array}$ & $\begin{array}{l}\text { high } \\
\text { high } \\
\text { high }\end{array}$ \\
\hline $\begin{array}{l}\text { Gaines et al. (1997, Study } 2 \text {, } \\
\text { Sample } 1 \text {, men) }\end{array}$ & $\begin{array}{l}\text { AfA (18) } \\
\text { AsA (46) } \\
\text { LtA (18) }\end{array}$ & 91 & 0 & CS & IND \& COL: Gaines et al. (1997) & $\begin{array}{r}-0.73 \\
0.18 \\
-0.57\end{array}$ & $\begin{array}{r}0.05 \\
-0.06 \\
0.16\end{array}$ & $\begin{array}{l}\text { low } \\
\text { low } \\
\text { low }\end{array}$ & $\begin{array}{l}\text { high } \\
\text { high } \\
\text { high }\end{array}$ \\
\hline $\begin{array}{l}\text { Gaines et al. (1997, Study } 2 \text {, } \\
\text { Sample 1, women) }\end{array}$ & $\begin{array}{l}\text { AfA (18) } \\
\text { AsA (46) } \\
\text { LtA (18) }\end{array}$ & 91 & 100 & CS & IND \& COL: Gaines et al. (1997) & $\begin{array}{l}-0.86 \\
-0.02 \\
-0.43\end{array}$ & $\begin{array}{l}-0.38 \\
-0.18 \\
-0.64\end{array}$ & $\begin{array}{l}\text { low } \\
\text { low } \\
\text { low }\end{array}$ & $\begin{array}{l}\text { high } \\
\text { high } \\
\text { high }\end{array}$ \\
\hline $\begin{array}{l}\text { Gaines et al. (1997, Study 2, } \\
\text { Sample 2, men) }\end{array}$ & $\begin{array}{l}\text { AfA (52) } \\
\text { AsA (36) } \\
\text { LtA (28) }\end{array}$ & 99 & 0 & $\mathrm{AD}$ & IND \& COL: Gaines et al. (1997) & $\begin{array}{r}0.00 \\
0.30 \\
-0.04\end{array}$ & $\begin{array}{l}-0.39 \\
-0.46 \\
-0.88\end{array}$ & $\begin{array}{l}\text { low } \\
\text { low } \\
\text { low }\end{array}$ & $\begin{array}{l}\text { high } \\
\text { high } \\
\text { high }\end{array}$ \\
\hline $\begin{array}{l}\text { Gaines et al. (1997, Study 2, } \\
\text { Sample 2, women) }\end{array}$ & $\begin{array}{l}\text { AfA (52) } \\
\text { AsA (36) } \\
\text { LtA (28) }\end{array}$ & 99 & 100 & $\mathrm{AD}$ & IND \& COL: Gaines et al. (1997) & $\begin{array}{r}-0.04 \\
-0.14 \\
0.49\end{array}$ & $\begin{array}{r}0.08 \\
-0.04 \\
0.06\end{array}$ & $\begin{array}{l}\text { low } \\
\text { low } \\
\text { low }\end{array}$ & $\begin{array}{l}\text { high } \\
\text { high } \\
\text { high }\end{array}$ \\
\hline Gaines et al. (1999, Study 1) & $\begin{array}{l}\text { AfA (4) } \\
\text { AsA (53) } \\
\text { LtA (4) }\end{array}$ & 45 & 64 & CS & IND \& COL: Gaines et al. (1997) & $\begin{array}{r}0.23 \\
0.31 \\
-0.61\end{array}$ & $\begin{array}{l}-1.55 \\
-1.67 \\
-1.56\end{array}$ & $\begin{array}{l}\text { low } \\
\text { low } \\
\text { low }\end{array}$ & $\begin{array}{l}\text { high } \\
\text { high } \\
\text { high }\end{array}$ \\
\hline Gaines et al. (1999, Study 2) & $\begin{array}{l}\text { AfA (16) } \\
\text { AsA (35) } \\
\text { LtA (27) }\end{array}$ & 170 & 64 & CS & IND \& COL: Gaines et al. (1997) & $\begin{array}{l}-0.13 \\
-0.05 \\
-0.11\end{array}$ & $\begin{array}{l}-0.25 \\
-0.19 \\
-0.36\end{array}$ & $\begin{array}{l}\text { low } \\
\text { low } \\
\text { low }\end{array}$ & $\begin{array}{l}\text { high } \\
\text { high } \\
\text { high }\end{array}$ \\
\hline Hetts et al. (1999, Study 1) & AsA (17) & 41 & - & CS & $\begin{array}{l}\text { IND \& COL: Kato \& Markus } \\
\text { (1993) }\end{array}$ & 0.52 & -0.44 & high & high \\
\hline Hetts et al. (1999, Study 2) & AsA (37) & 70 & - & CS & $\begin{array}{l}\text { IND \& COL: Kato \& Markus } \\
\text { (1993) }\end{array}$ & 0.34 & -0.51 & high & high \\
\hline Lum (1997) & AsA (186) & 91 & 61 & CS & IND \& COL: Singelis et al. (1995) & 0.12 & -0.29 & high & low \\
\hline
\end{tabular}


Appendix B (continued)

\begin{tabular}{|c|c|c|c|c|c|c|c|c|c|}
\hline \multirow[b]{2}{*}{ Study } & \multirow[b]{2}{*}{$\begin{array}{l}\text { Comparison } \\
\text { groups }(n)\end{array}$} & \multirow[b]{2}{*}{$\begin{array}{c}\text { European } \\
\text { American }(n)\end{array}$} & \multirow[b]{2}{*}{$\begin{array}{l}\text { Overall \% } \\
\text { female }^{\mathrm{a}}\end{array}$} & \multirow[b]{2}{*}{ Population } & \multirow[b]{2}{*}{ IND/COL measure } & \multicolumn{2}{|c|}{ Effect size $(d)$} & \multicolumn{2}{|c|}{ Reliability } \\
\hline & & & & & & IND & $\mathrm{COL}$ & IND & $\mathrm{COL}$ \\
\hline Matsumoto et al. (1997, Study 6) & $\begin{array}{l}\text { AfA (21) } \\
\text { AsA (84) } \\
\text { LtA (32) }\end{array}$ & 114 & 73 & $\mathrm{CS}$ & COL: Matsumoto et al. (1997) & & $\begin{array}{l}-0.14 \\
-0.64 \\
-0.69\end{array}$ & & $\begin{array}{l}\text { high } \\
\text { high } \\
\text { high }\end{array}$ \\
\hline Oishi et al. (1998, Sample 1) & $\begin{array}{l}\text { AfA (6) } \\
\text { AsA (17) } \\
\text { LtA (9) }\end{array}$ & 110 & - & $\mathrm{CS}$ & $\begin{array}{l}\text { IND \& COL: Singelis (1994); } \\
\text { Singelis et al. (1995) }\end{array}$ & $\begin{array}{r}-0.45 \\
0.04 \\
-0.13\end{array}$ & $\begin{array}{r}-0.11 \\
-0.38 \\
0.24\end{array}$ & $\begin{array}{l}\text { low } \\
\text { low } \\
\text { low }\end{array}$ & $\begin{array}{l}\text { low } \\
\text { low } \\
\text { low }\end{array}$ \\
\hline Oishi et al. (1998, Sample 2) & $\begin{array}{l}\text { AfA (3) } \\
\text { AsA (19) } \\
\text { LtA (4) }\end{array}$ & 83 & - & $\mathrm{CS}$ & IND \& COL: Singelis (1994) & $\begin{array}{r}-0.97 \\
0.10 \\
-0.64\end{array}$ & $\begin{array}{r}1.35 \\
-0.26 \\
0.12\end{array}$ & $\begin{array}{l}\text { low } \\
\text { low } \\
\text { low }\end{array}$ & $\begin{array}{l}\text { low } \\
\text { low } \\
\text { low }\end{array}$ \\
\hline Okazaki (1997) & AsA (165) & 183 & 54 & $\mathrm{CS}$ & IND \& COL: Takata (1993) & $\begin{array}{r}0.39 \\
-0.58\end{array}$ & -0.58 & - & \\
\hline Okazaki (2000) & AsA (39) & 42 & $56(69)$ & CS & IND \& COL: Singelis (1994) & 0.49 & 0.60 & - & \\
\hline Oyserman, Gant, \& Ager (1995) & AfA (32) & 57 & $84(63)$ & $\mathrm{CS}$ & IND \& COL: Oyserman (1993) & -0.04 & -0.43 & low & low \\
\hline Rhee et al. (1996) & AsA (140) & 133 & $65(59)$ & CS & $\begin{array}{l}\text { IND \& COL: Triandis (1991), } \\
\text { Yamaguchi (1994), Hui (1988) }\end{array}$ & -0.01 & -0.15 & - & \\
\hline Singelis (1994, Sample 1) & $\begin{array}{l}\text { AfA (7) } \\
\text { AsA (208) }\end{array}$ & 49 & 57 & $\mathrm{CS}$ & IND \& COL: Singelis (1994) & $\begin{array}{l}0.55 \\
0.41\end{array}$ & $\begin{array}{r}0.16 \\
-0.41\end{array}$ & $\begin{array}{l}\text { high } \\
\text { high }\end{array}$ & $\begin{array}{l}\text { high } \\
\text { high }\end{array}$ \\
\hline Singelis (1994, Sample 2) & AsA (95) & 300 & 57 & $\mathrm{CS}$ & IND \& COL: Singelis (1994) & 0.41 & -0.41 & high & high \\
\hline Singelis \& Sharkey (1995) & AsA (417) & 86 & 56 & $\mathrm{CS}$ & IND \& COL: Singelis (1994) & 0.65 & -0.48 & high & high \\
\hline $\begin{array}{l}\text { Singelis, Bond, Sharkey, \& Lai } \\
\text { (1999) }\end{array}$ & AsA (146) & 232 & $66(38)$ & CS & IND \& COL: Singelis et al. (1995) & 0.58 & -0.57 & low & low \\
\hline Singelis et al. (1995) & $\begin{array}{l}\text { AsA (72) } \\
\text { LtA (3) }\end{array}$ & 68 & - & $\mathrm{CS}$ & IND \& COL: Singelis et al. (1995) & $\begin{array}{r}0.13 \\
-0.24\end{array}$ & $\begin{array}{r}-0.42 \\
0.17\end{array}$ & $\begin{array}{l}\text { low } \\
\text { low }\end{array}$ & $\begin{array}{l}\text { low } \\
\text { low }\end{array}$ \\
\hline Whatley (1997) & $\begin{array}{l}\text { AfA (33) } \\
\text { AsA (16) }\end{array}$ & 356 & 50 & CS & IND \& COL: Singelis et al. (1995) & $\begin{array}{l}-0.55 \\
-0.02\end{array}$ & $\begin{array}{r}0.07 \\
-0.16\end{array}$ & $\begin{array}{l}\text { high } \\
\text { low }\end{array}$ & $\begin{array}{l}\text { high } \\
\text { low }\end{array}$ \\
\hline
\end{tabular}

Note. Dashes indicate value is not available. $\mathrm{IND}=$ individualism; $\mathrm{COL}=$ collectivism; $\mathrm{AfA}=\mathrm{African}$ Americans; $\mathrm{AsA}=\mathrm{Asian} \mathrm{Americans} ; \mathrm{LtA}=\mathrm{Latino} \mathrm{Americans} ; \mathrm{CS}=$ college students; GS = graduate students; $\mathrm{AD}=$ adults.

${ }^{a}$ Number in parentheses in this column is the overall percentage of European American participants. 


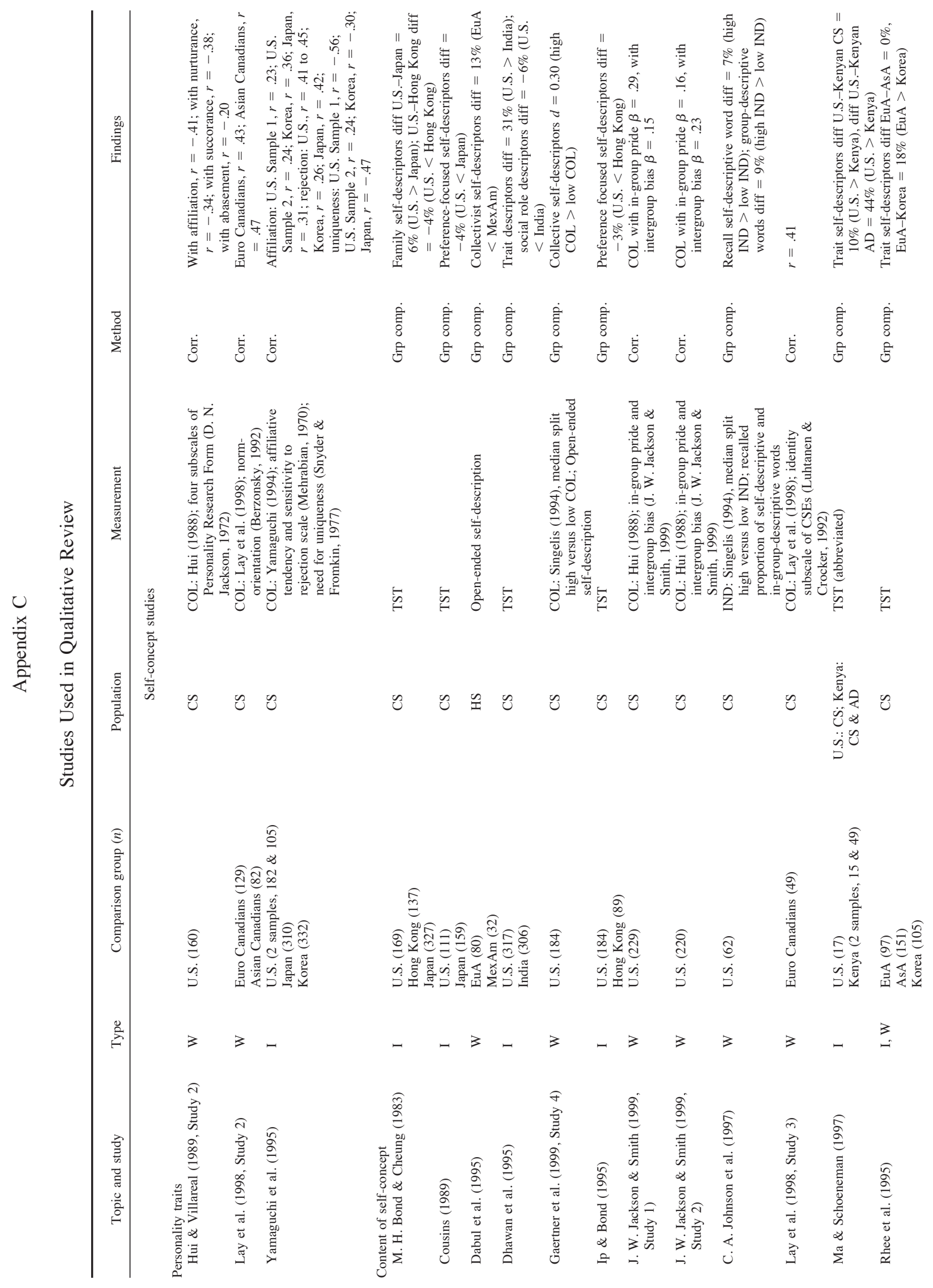




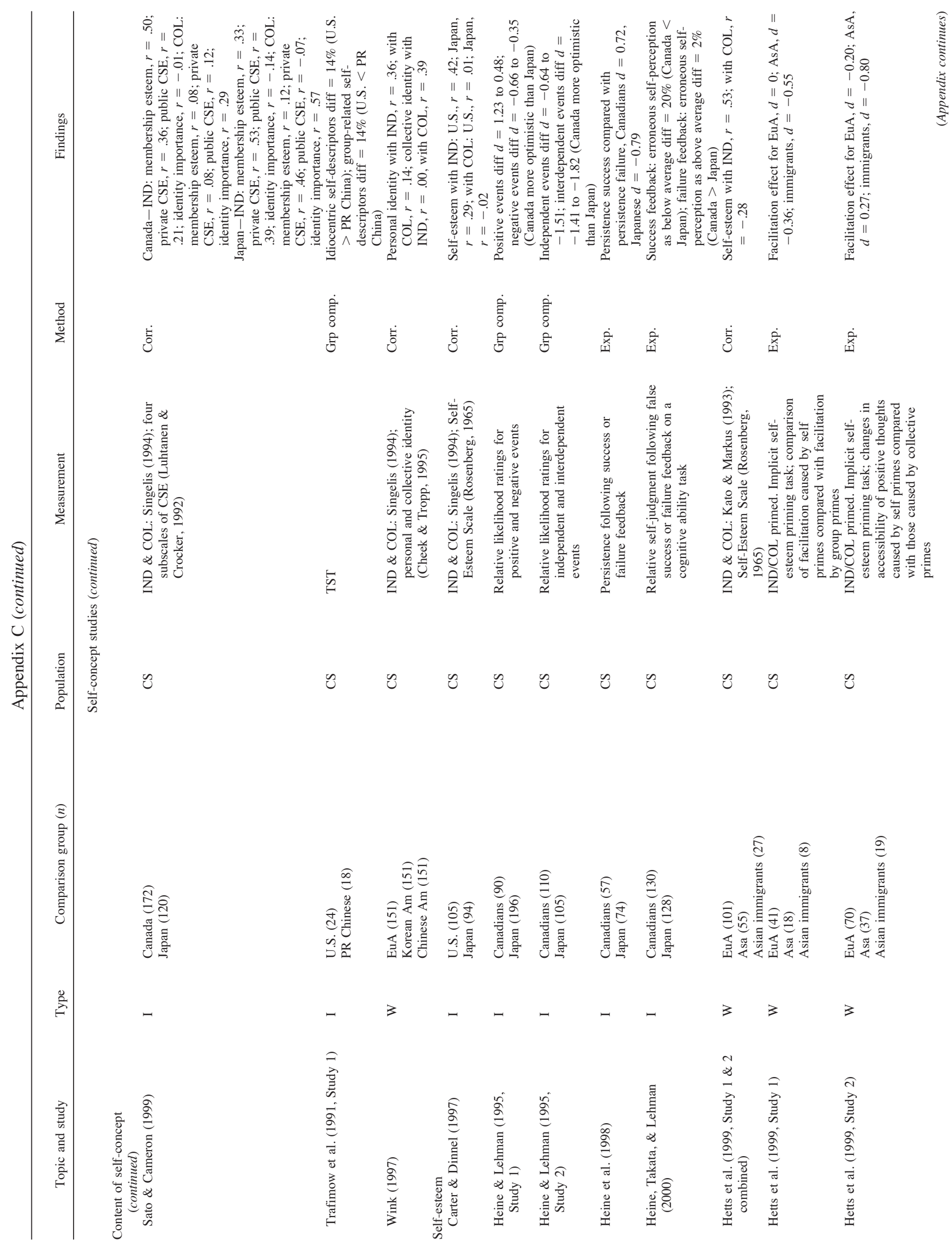




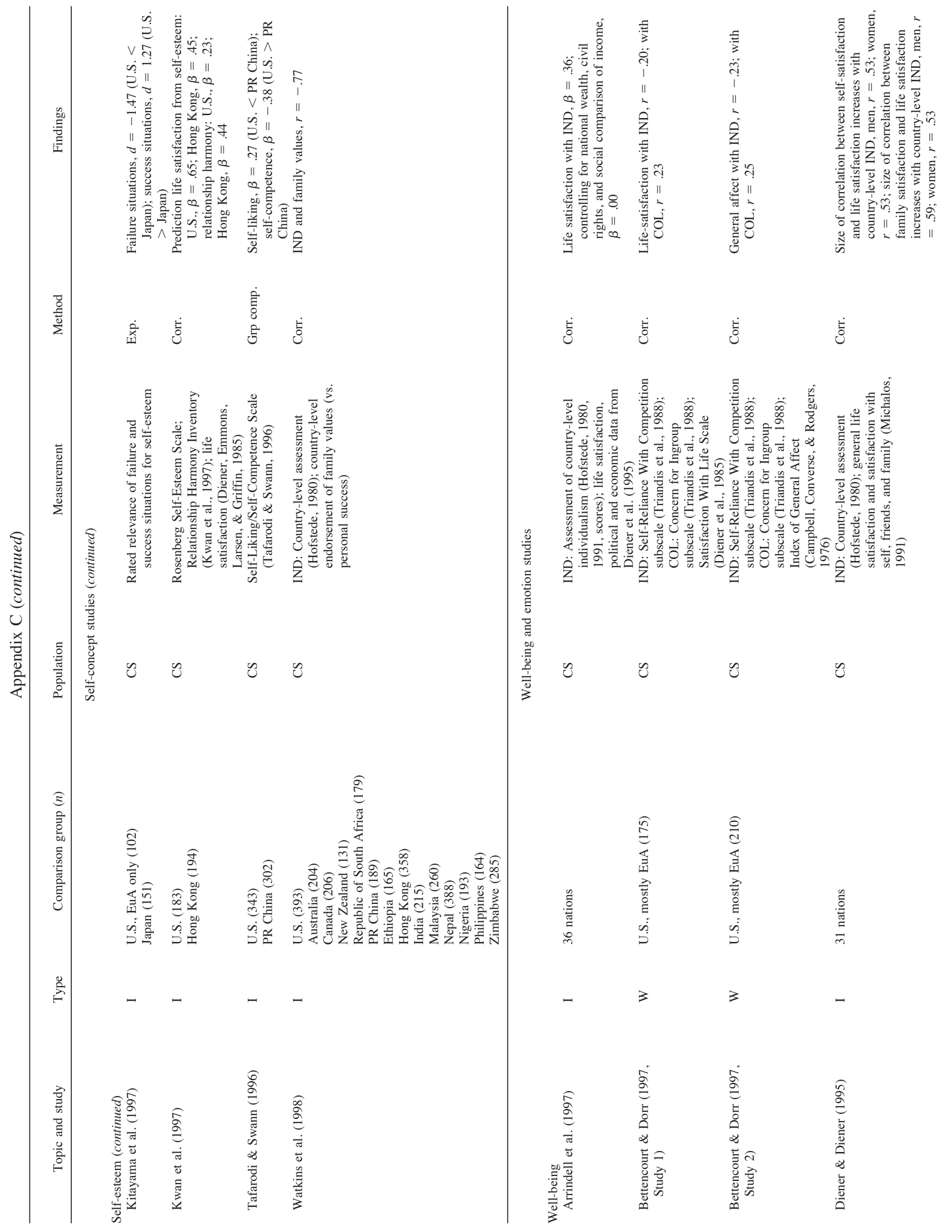




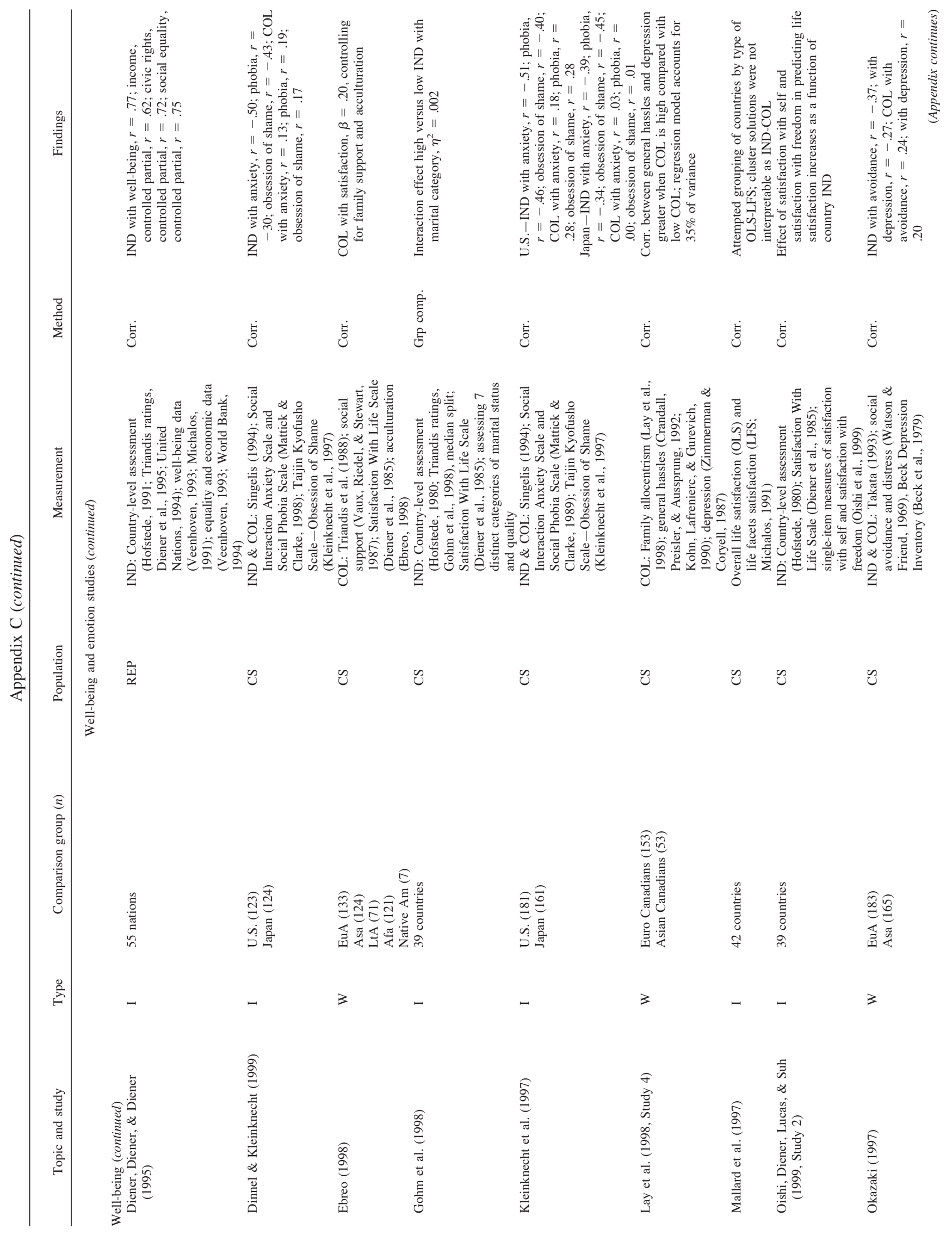




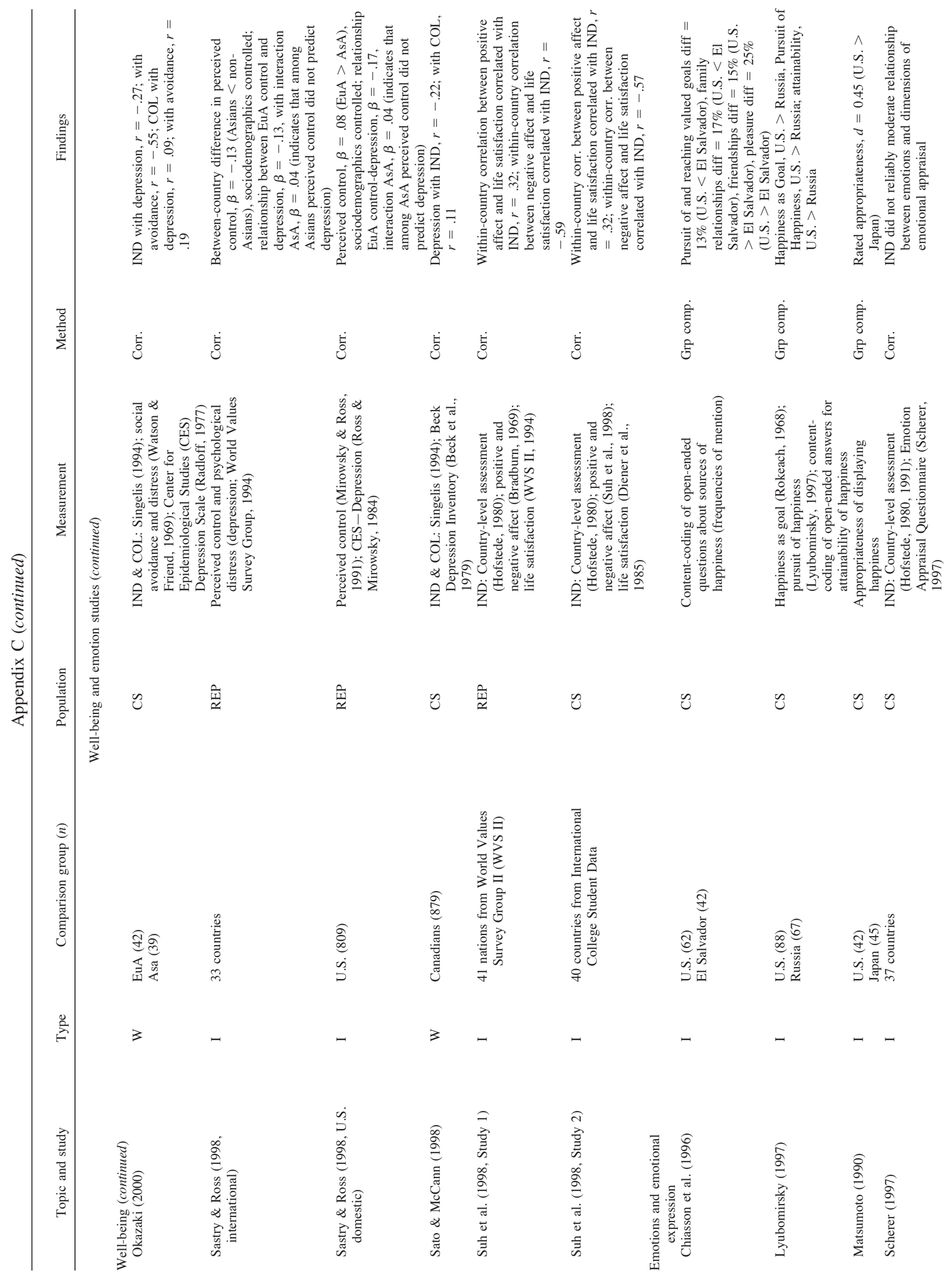




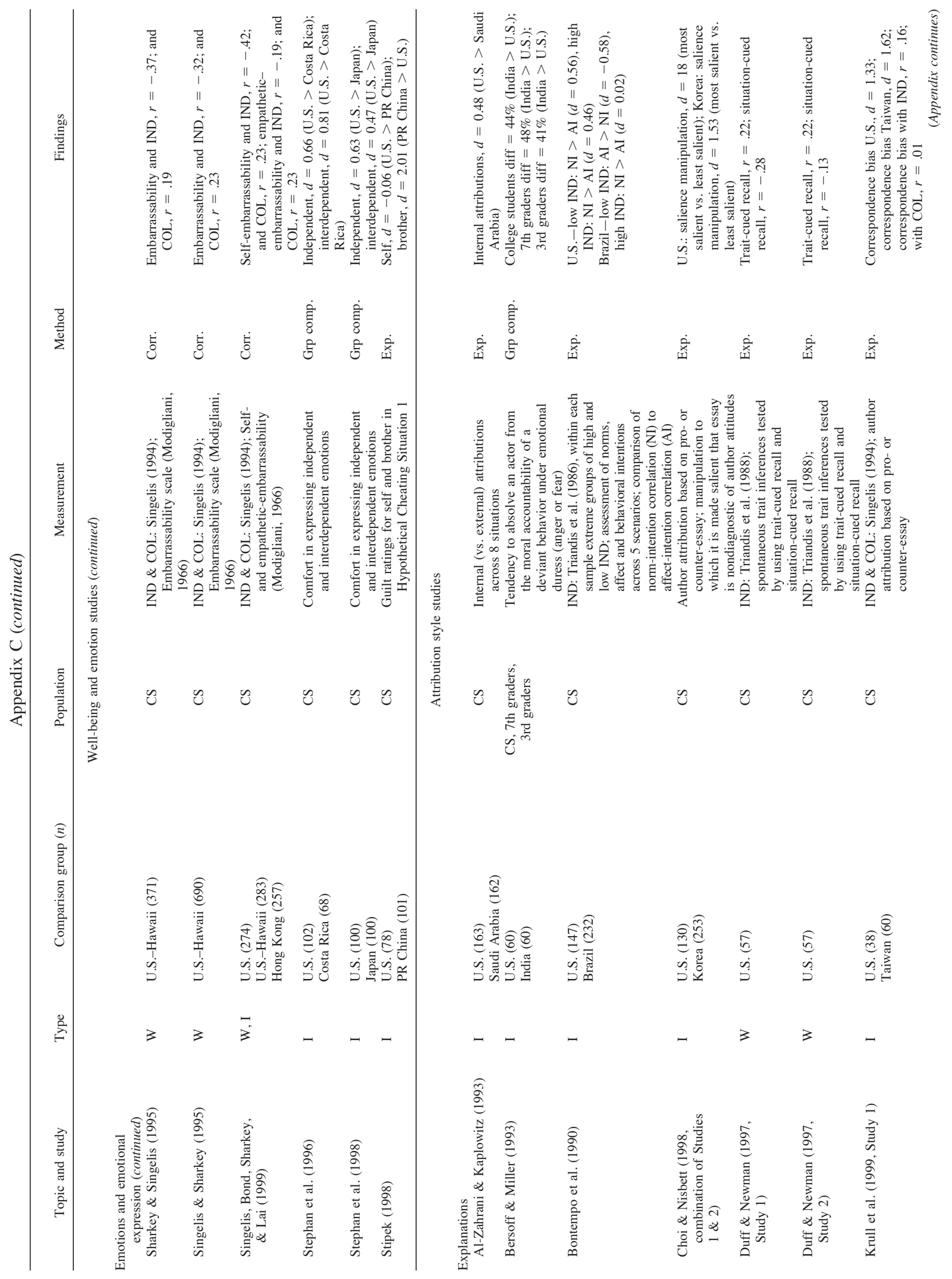




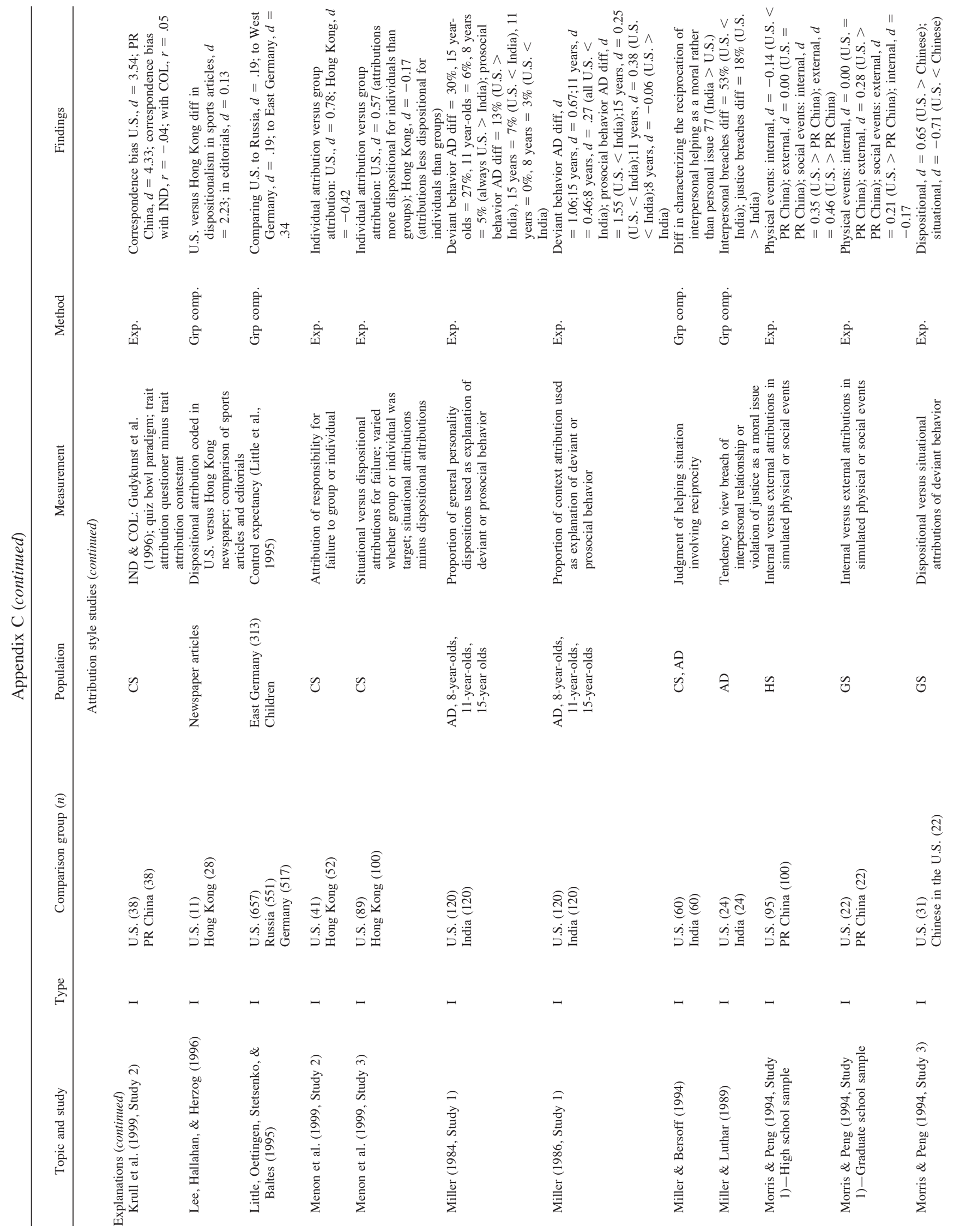




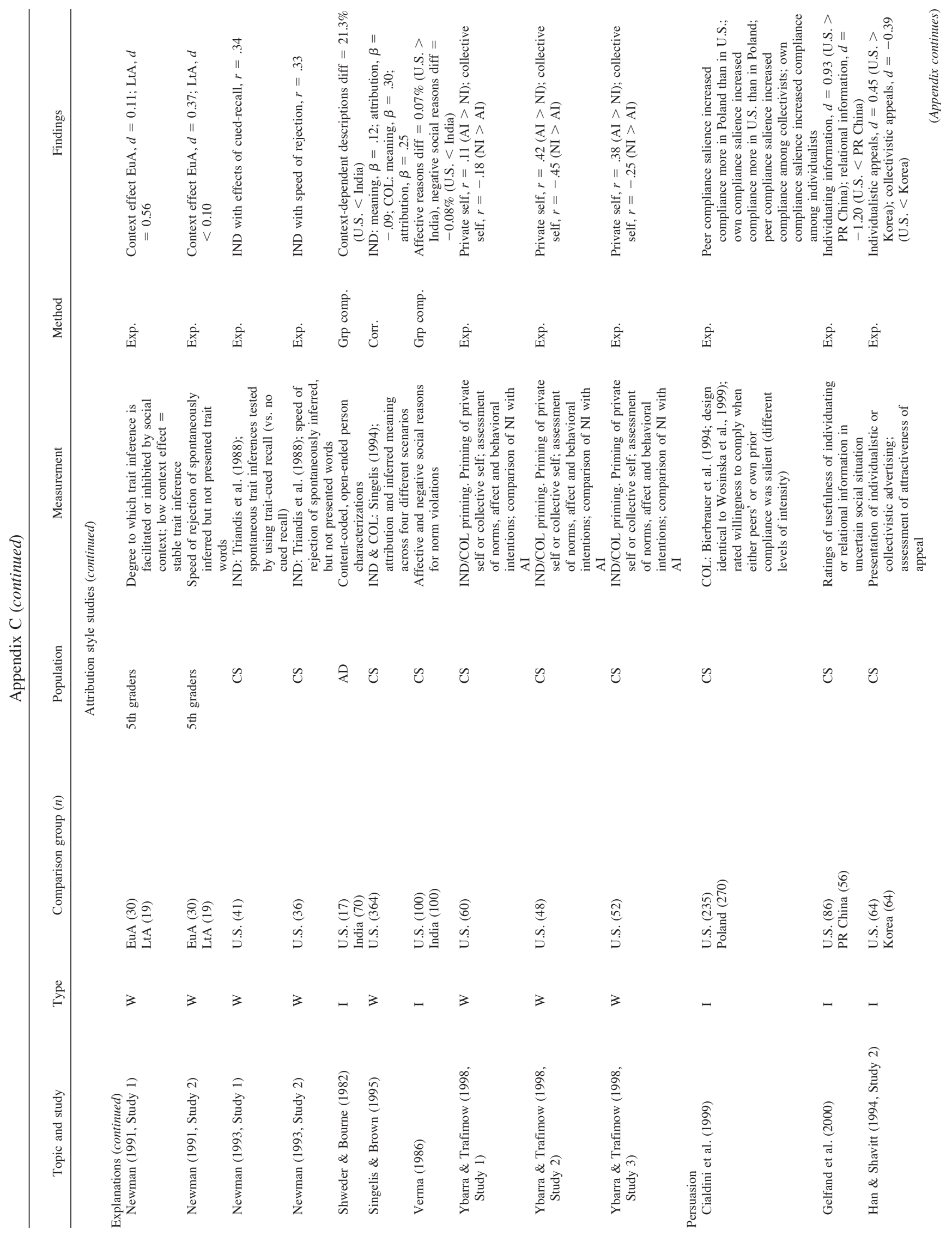




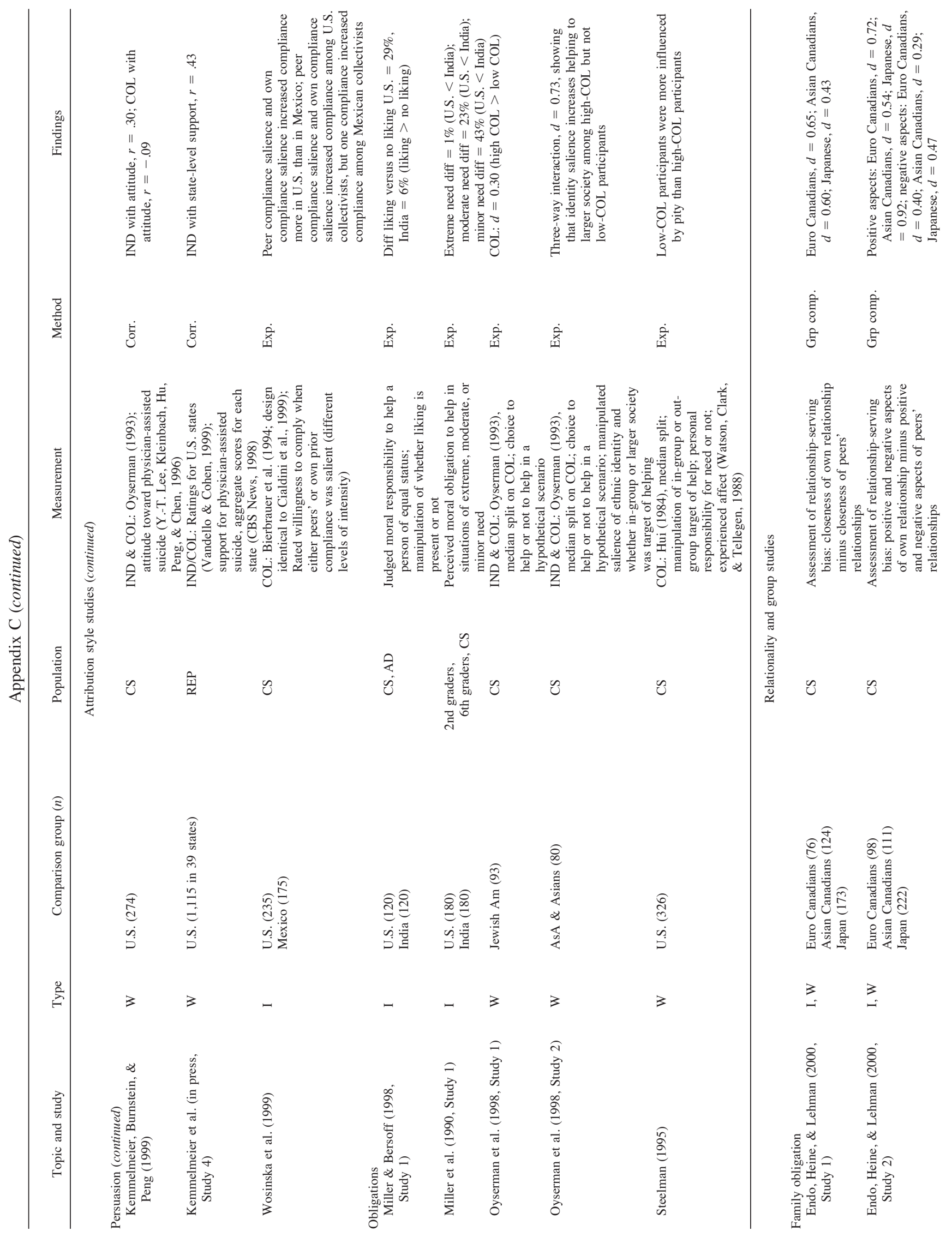




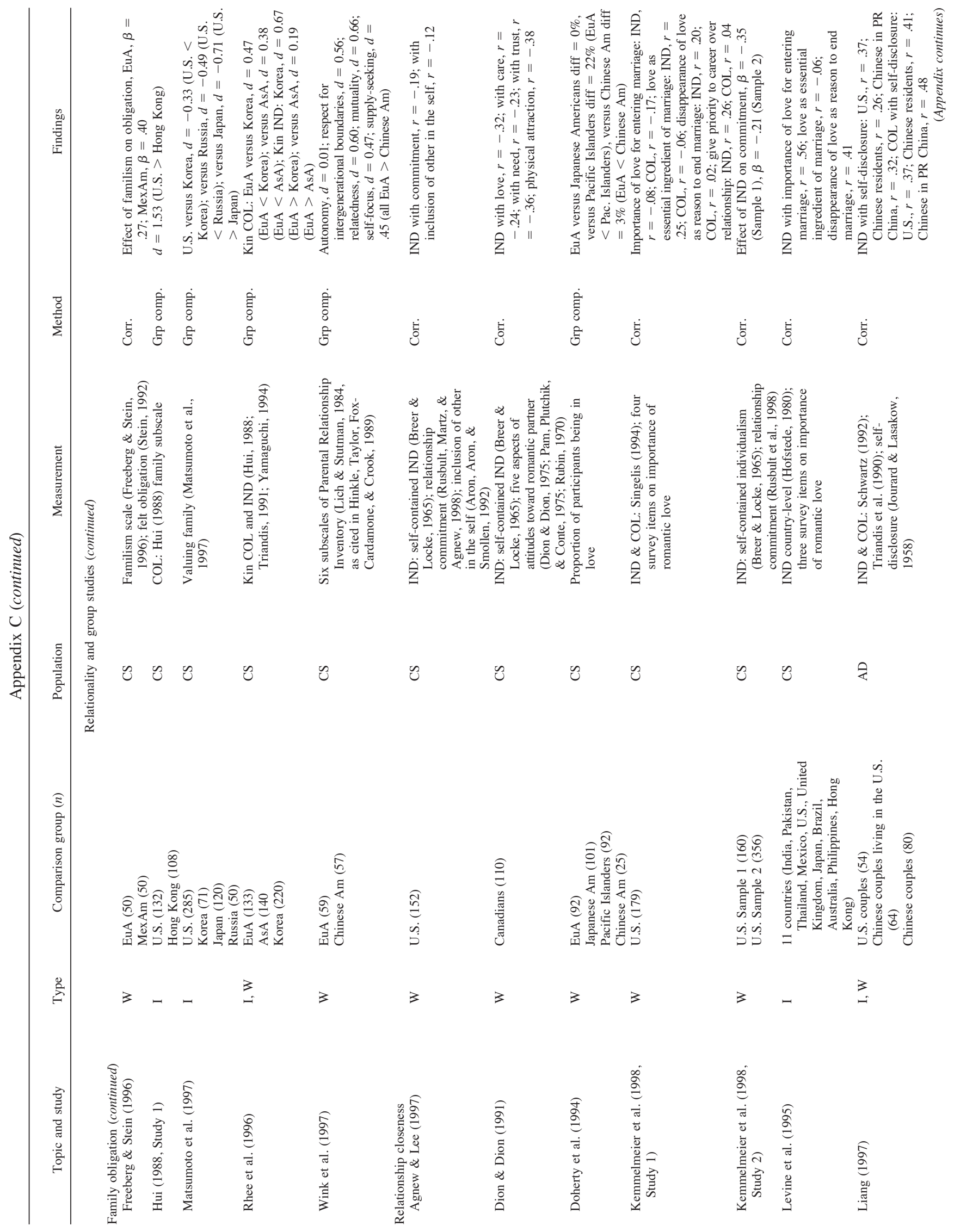




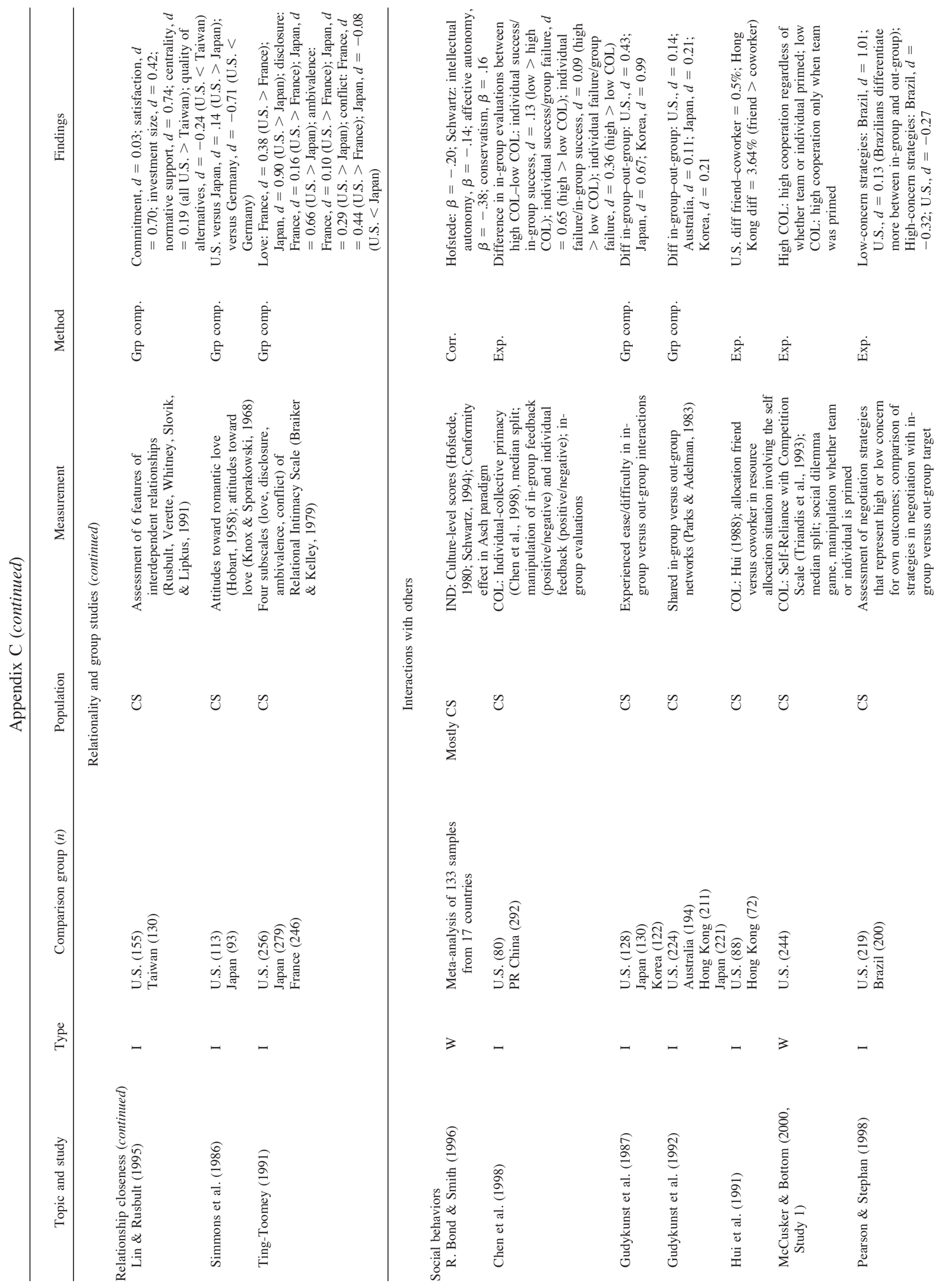


INDIVIDUALISM AND COLLECTIVISM

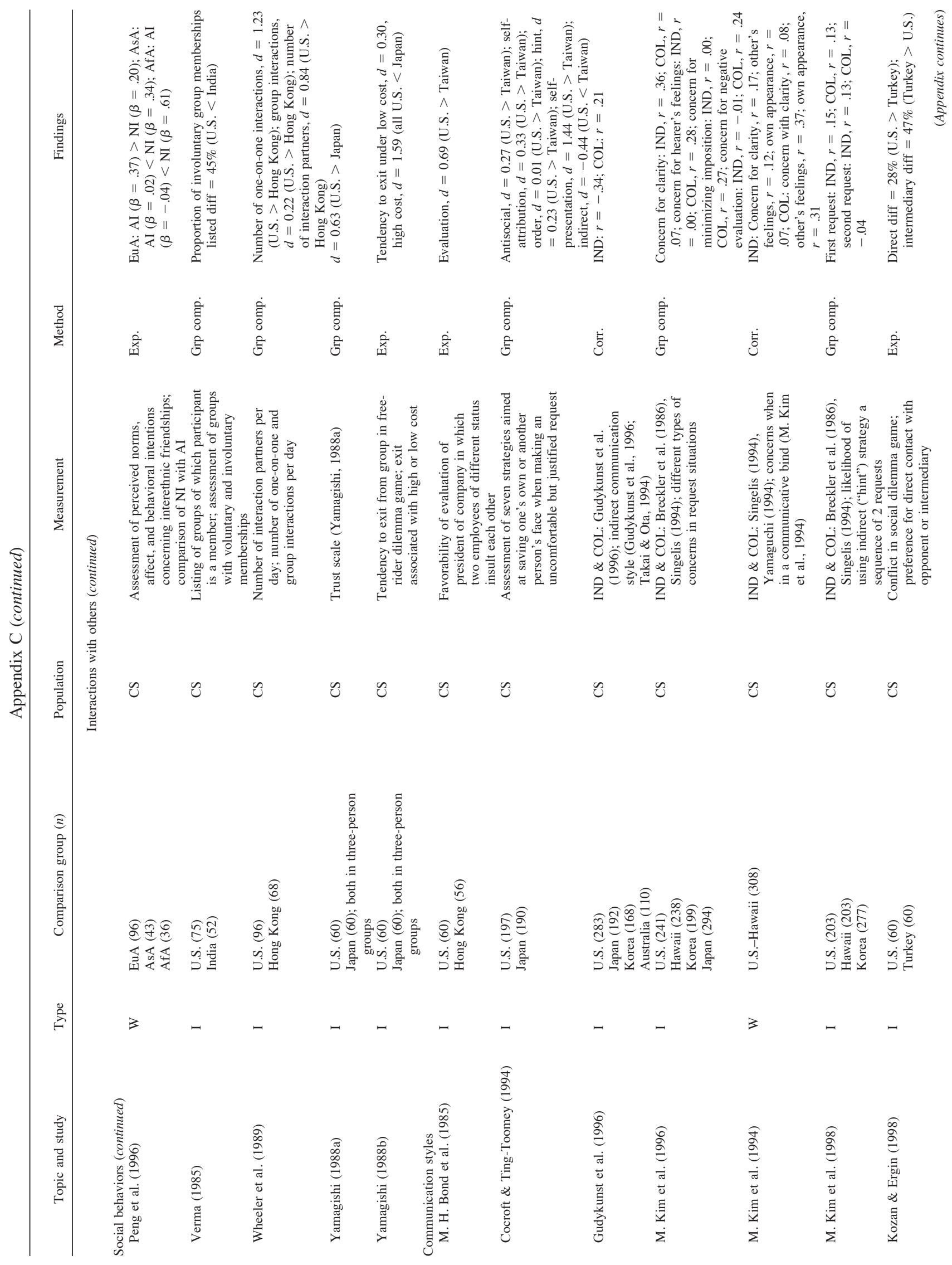




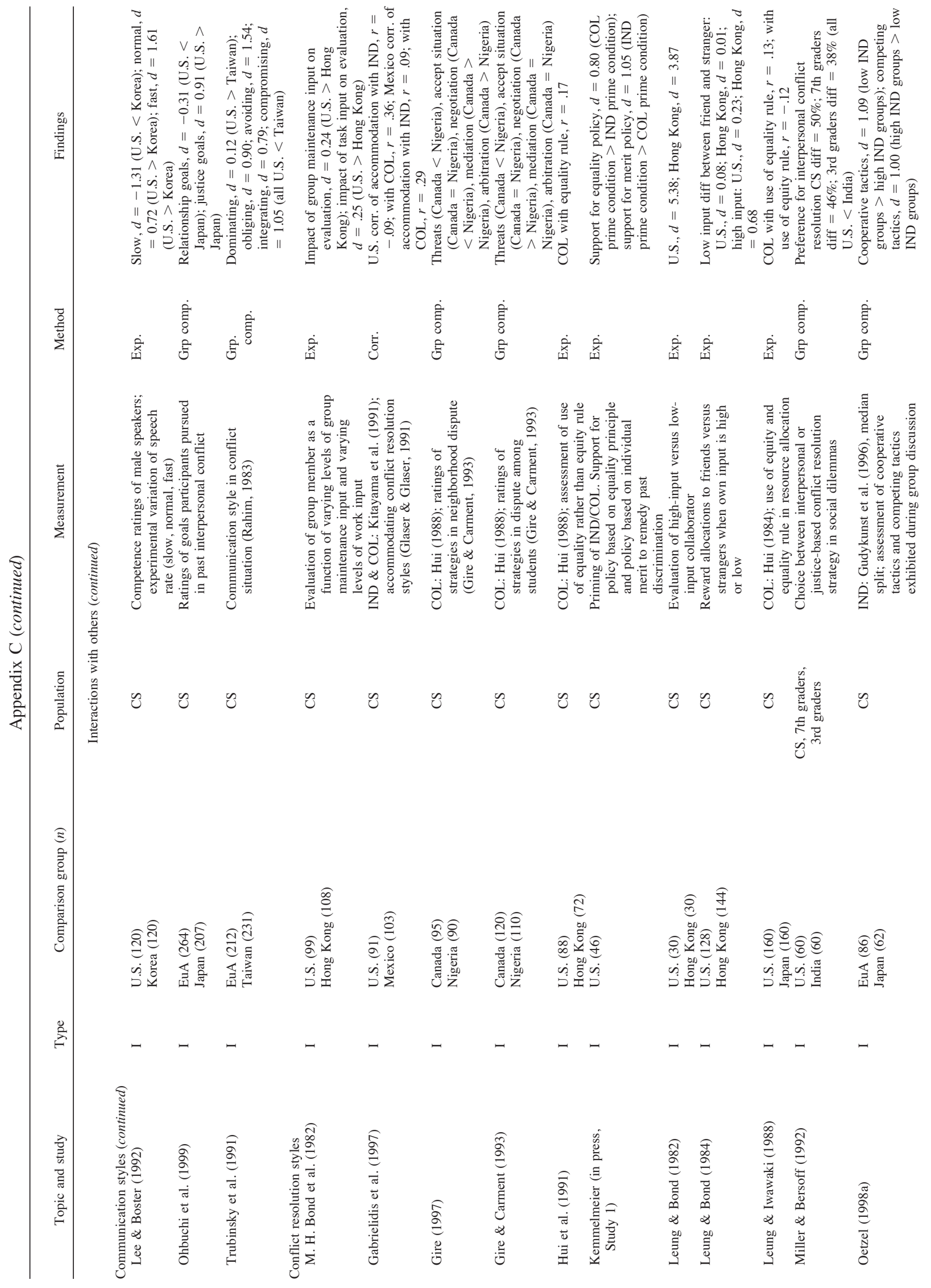


INDIVIDUALISM AND COLLECTIVISM

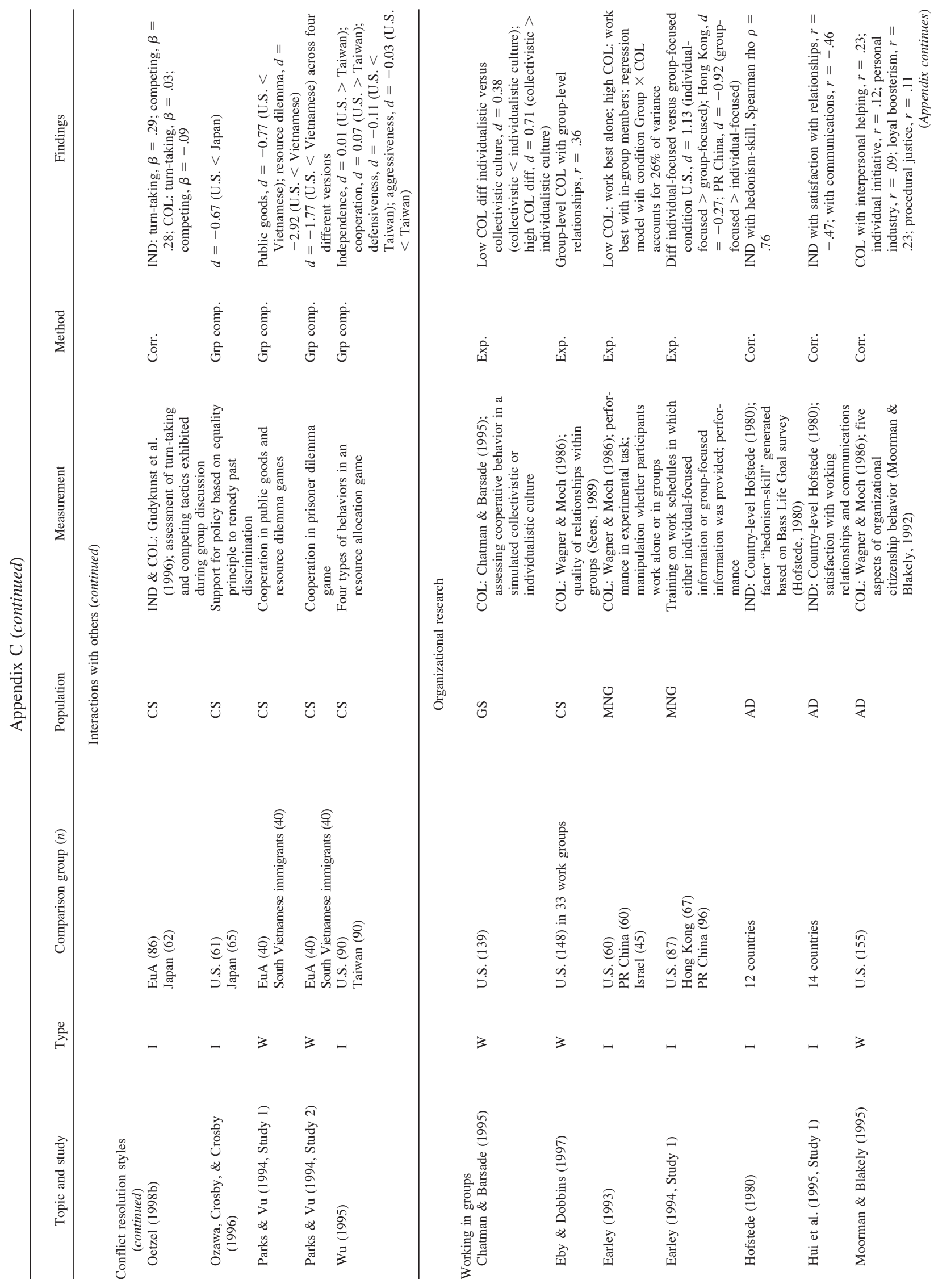




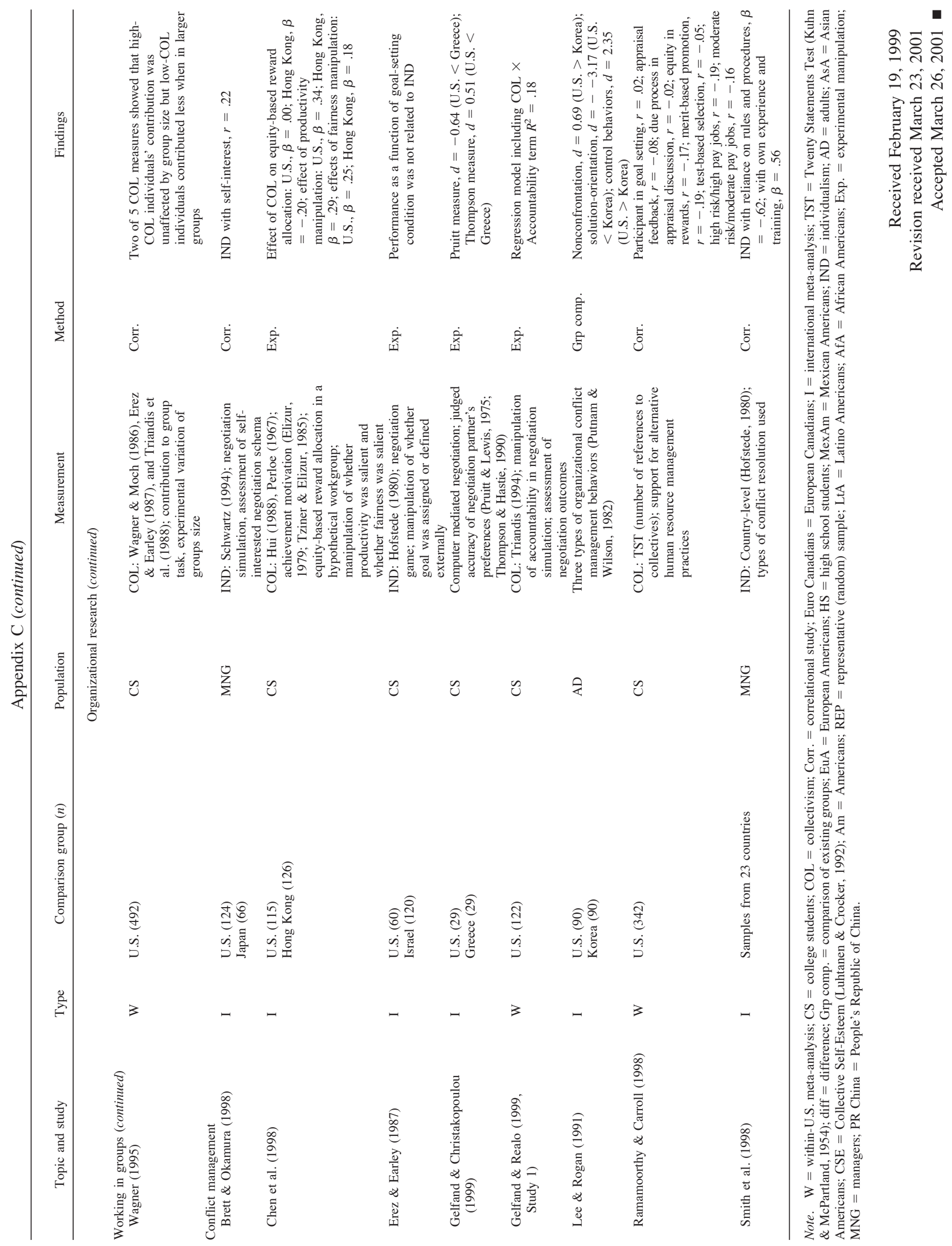

